\title{
WestVirginiaUniversity
}

THE RESEARCH REPOSITORY @ WVU

Graduate Theses, Dissertations, and Problem Reports

2010

\section{Pulsar Populations in the Galaxy and Magellanic Clouds}

Joshua P. Ridley

West Virginia University

Follow this and additional works at: https://researchrepository.wvu.edu/etd

\section{Recommended Citation}

Ridley, Joshua P., "Pulsar Populations in the Galaxy and Magellanic Clouds" (2010). Graduate Theses, Dissertations, and Problem Reports. 3204.

https://researchrepository.wvu.edu/etd/3204

This Dissertation is protected by copyright and/or related rights. It has been brought to you by the The Research Repository @ WVU with permission from the rights-holder(s). You are free to use this Dissertation in any way that is permitted by the copyright and related rights legislation that applies to your use. For other uses you must obtain permission from the rights-holder(s) directly, unless additional rights are indicated by a Creative Commons license in the record and/ or on the work itself. This Dissertation has been accepted for inclusion in WVU Graduate Theses, Dissertations, and Problem Reports collection by an authorized administrator of The Research Repository @ WVU.

For more information, please contact researchrepository@mail.wvu.edu. 


\title{
Pulsar Populations in the Galaxy and Magellanic Clouds
}

\author{
Joshua P. Ridley \\ Dissertation submitted to the \\ Eberly College of Arts and Sciences \\ at West Virginia University \\ in partial fulfillment of the requirements \\ for the degree of \\ Doctor of Philosophy \\ in \\ Physics \\ Dr. Duncan Lorimer, Ph.D., Chair \\ Dr. Maura McLaughlin, Ph.D. \\ Dr. John Littleton, Ph.D. \\ Dr. Boyd Edwards, Ph.D. \\ Dr. Matthew Valenti, Ph.D. \\ Department of Physics \\ Morgantown, West Virginia \\ 2010
}

Keywords: Pulsars; Neutron Stars Copyright 2010 Joshua P. Ridley 


\title{
ABSTRACT \\ Pulsar Populations in the Galaxy \\ and \\ Magellanic Clouds
}

\author{
Joshua P. Ridley
}

Pulsars are great tools for studying the Universe. From energetics to radio emission, many of the properties of pulsars can be used to understand other physical phenomena occurring throughout the Universe today. This dissertation examines pulsars and their properties through the use of computer simulations, describes the basic pulsar search procedure, and presents the results of a search for millisecond pulsars (MSPs) and fast radio transients in the Small Magellanic Cloud (SMC).

Using Monte Carlo simulations, a working model of normal pulsars that accurately represents the observed population was created. These simulations allow for changes to be made to the initial spin period, luminosity, braking index, and magnetic field distributions (among others), and the resulting model can be compared to the observed sample of pulsars. These simulations have shown that the luminosity must have some dependence on the spin period and that the braking index has very little effect on the overall pulsar population. Also, it was found to be not possible to produce a self-consistent model of the pulsar population that includes magnetic axis alignment in any of the currently known spin-down laws.

A practical use of these models is the prediction of the results of future pulsar surveys. The model populations can be used to determine the number of pulsars that could be found in a similar survey done with a real telescope. For the purposes of this dissertation, the pulsar population of both the Large and Small Magellanic Clouds (LMC and SMC) were simulated and then searched for pulsars. After accounting for the low escape velocities of both Clouds, the estimated birthrate of pulsars in either Cloud is $0.5-1.0$ pulsars per century, and the estimated numbers of normal pulsars are $(1.79 \pm 0.20) \times 10^{4}$ and $(1.09 \pm 0.16) \times 10^{4}$ for the LMC and SMC, respectively. For the MSP populations, upper limits of 15,000 and 23,000 for the LMC and SMC were obtained. The SMC simulations predicted that up to three MSPs could be detected in a new, high-resolution survey performed at the Parkes Telescope in Parkes, Australia.

The pulsar search procedure is mostly computerized and is very data intensive. Fourier transforms are utilized in the search for periodic signals, and matched filtering is used to search for single bursts of radio emission. Acceleration searches attempt to find pulsars that are locked in binary orbits with other stars in which the pulsars' accelerations significantly affect the timing of the signals that telescopes 
on the Earth detect. These search techniques were applied to a survey for pulsars in the SMC that was executed at the Parkes Telescope in Parkes, Australia. This survey has, to date, redetected three known pulsars, discovered three potential MSP candidates, and placed an upper limit of 15 bursting sources in the SMC. The results of this survey have now led to a similar survey of the Large Magellanic Cloud that is currently in progress. 


\section{Acknowledgments}

Numerous people have enabled me to successfully survive my graduate career here at West Virginia University. Graduate classes, research projects, and even social events would not have been as fun or as purposeful without the support and encouragement from everyone in Morgantown who decided to help me and come along with me on this journey.

I first must thank Dr. Duncan Lorimer for supervising all of my research projects and teaching me much of what I now know about astrophysics, radio astronomy, and, of course, pulsars. Perhaps the most important lesson I have learned is the art of not giving up when you hit a dead end, which Dunc has (possibly unknowingly) taught me by always being able to view my problems from different angles and by attacking them with alternative (although sometimes more complicated) methods.

A great deal of thanks must be extended to my other committee members, Drs. Maura McLaughlin, John (Jack) Littleton, Boyd Edwards, and Matthew Valenti. Their time and effort in reviewing this work and providing insightful comments is greatly appreciated.

Specific thank yous need to be given to Vlad Kondratiev, Joris Verbiest, Paulo Freire, Froney Crawford, and John Sarkissian for providing computing, $\mathrm{AT}_{\mathrm{E}} \mathrm{X}$, and observational support.

I especially want to thank the Physics Department at West Virginia University. Dr. Earl Scime, Sherry Puskar, Siobhan Byrne, Devon Cleland, Phil Tucker, and Sandy Johns have been helpful in many different areas and I certainly would have had a much more difficult and hectic time here without them.

A special thank you is warranted to my fellow pulsar graduate students from the windowless office of 111 for putting up with me as an office mate through the years, so thank you, Ben, Jason, Josh, Mitch, and Priya.

I am incredibly grateful for my parents, Paul and Mary, for supporting me on the long road down which my education has taken me and for my siblings, Justin, Micah, and Libby. Without all of them I most certainly would not be who or where I am today. I also want to thank God for giving me the ability to learn, presenting me with opportunities that I have had throughout my life, and for giving me the courage and wisdom to handle everything that has been thrown my way.

Finally, I want to thank my wonderful wife, Elizabeth, for supporting me through all of the ups and downs associated with being a graduate student, a husband, and a father. She is an amazing mommy to our son, Jacob, and I am dedicating this work to our family. 


\section{Table of Contents}

List of Tables viii

List of Figures $\quad$ ix

List of Abbreviations and Symbols $\quad \mathrm{X}$

1 Introduction 1

1.1 A Brief History of Pulsars . . . . . . . . . . . . . . . . . . . . 1

1.2 Pulsars Unfurled . . . . . . . . . . . . . . . . . . 4

1.2.1 Rotation Rates . . . . . . . . . . . . . . . . . . 4

1.2.2 Rotational Kinetic Energy . . . . . . . . . . . . . . . . 6

1.2 .3 Magnetic Field . . . . . . . . . . . . . . . . . . . 7

1.2.4 Braking Index and Spin-down . . . . . . . . . . . . . 8

1.2.5 Evolution on the $P-\dot{P}$ Diagram . . . . . . . . . . . . . . 9

1.2.6 Dispersion Measure . . . . . . . . . . . . . . . . . . . . 9

1.2 .7 Scattering . . . . . . . . . . . . . . . . 11

1.2.8 Luminosity and Flux Density . . . . . . . . . . . . . 14

1.3 Outline of Dissertation . . . . . . . . . . . . . . . . . . . 15

2 Isolated Pulsar Spin Evolution on the $P-\dot{P}$ Diagram 18

2.1 Introduction . . . . . . . . . . . . . . . . . . . 18

2.2 Modeling the Underlying Pulsar Population . . . . . . . . . . . . . 20

2.3 Modeling the Observed Pulsar Population . . . . . . . . . . . . . . . 28

2.4 Method for Comparison ........................ 30

2.5 Results and Discussion . . . . . . . . . . . . . . . . . 31

2.5.1 Simulation Models ... . . . . . . . . . . . . 32

2.5.1.1 Model 1: FK06 Basic Parameters . . . . . . . . . 33

2.5.1.2 Model 2: Modified Luminosity Law and Random Inclination Angles . . . . . . . . . . . . . . 36

2.5.1.3 Model 3: Beaming Model and Braking Index Variations . . . . . . . . . . . . . . 37

2.5.1.4 Model 4: Inclination Angle Decay . . . . . . . . . . . 37

2.5.2 Individual Parameters . . . . . . . . . . . . . . . 39

2.5.2.1 Beaming................... 39

2.5.2.2 Braking Index . . . . . . . . . . . . . . . . . 40

2.5.2.3 Luminosity . . . . . . . . . . . . . . . 40

2.5.2.4 Covariances .................. . . . . 42

2.5.2.5 Death Line ................ . . . 42

2.6 Conclusions . . . . . . . . . . . . . . . . . . . . . . . . 43 
3 Computer Simulations of the Magellanic Clouds Pulsar Population 45

3.1 Introduction . . . . . . . . . . . . . . . . . . . . 45

3.2 Previous Surveys of the Magellanic Clouds . . . . . . . . . . . . . 46

3.3 Modeling the Normal Pulsar Population . . . . . . . . . . . . . 48

3.4 Modeling the Millisecond Pulsar Population . . . . . . . . . . . . . 51

3.5 Discussion . . . . . . . . . . . . . . . . . 53

3.5.1 Birthrates of Normal Pulsars . . . . . . . . . . . . . 53

3.5.2 Predictions for Future Surveys . . . . . . . . . . . . 55

3.6 Conclusions ......................... 56

4 Searching for Pulsars $\quad 59$

4.1 Introduction . . . . . . . . . . . . . . . . . . 59

4.2 Obtaining and Formatting the Data . . . . . . . . . . . . . . . 59

4.2.1 Barycentering ...................... 60

4.2.2 De-dispersion .................... . . . . 62

4.3 Periodicity Searches . . . . . . . . . . . . . . . . . 65

4.4 Single Pulse Searches . . . . . . . . . . . . . . . . . 70

4.4.1 Matched Filtering . . . . . . . . . . . . . . . . 71

4.4.2 Radio Frequency Interference . . . . . . . . . . . . . . . . . 73

4.5 Acceleration Searches . . . . . . . . . . . . . . . . 73

5 A Survey for Pulsars and Fast Transients in the Small Magellanic Cloud 79

5.1 Introduction . . . . . . . . . . . . . . . . . . . 79

5.2 Observations . . . . . . . . . . . . . . . . . . . 81

5.3 Results............................. 84

5.3.1 Known Pulsars ....................... 84

5.3.1.1 PSR J0113-7220 . . . . . . . . . . . . . . . 86

$5.3 .1 .2 \quad$ PSR J0131-7310 . . . . . . . . . . . . . . . 87

5.3.1.3 PSR J0057-7201 . . . . . . . . . . . . . 88

5.3.2 MSP Candidates . . . . . . . . . . . . . . . . 89

5.3.2.1 SMC J0042-7300 . . . . . . . . . . . . . . . 89

5.3 .2 .2 SMC J0045-7300 . . . . . . . . . . . . . 90

5.3.2.3 SMC J0111-7350 . . . . . . . . . . . . . . . . 91

5.3.2.4 Follow-up Observations ............. 91

5.4 Discussion . . . . . . . . . . . . . . . . . . . . . . 92

5.4.1 Candidates Versus Known Pulsars . . . . . . . . . . . . . . . . 92

5.4 .2 Expected S/N . . . . . . . . . . . . . . . . . 92

5.4 .3 Single Pulses . . . . . . . . . . . . . . . . 93

5.4 Survey Limitations . . . . . . . . . . . . . . . 96

5.5 Conclusions . . . . . . . . . . . . . . . . . . . . . . . . . 99

6 Conclusions 100

A An Open-source Approach to Pulsar Population Synthesis 107 
B A Search for the Youngest Pulsar in the Galaxy 


\section{List of Tables}

2.1 KS Comparison of Rotational Period Distributions . . . . . . . . . . 30

2.2 Figure of Merit Comparison of Simulated Models . . . . . . . . . . . 36

2.3 Braking Index Variations . . . . . . . . . . . . . . . . . 41

3.1 SMC Survey Parameters . . . . . . . . . . . . . . . . . . 46

3.2 List of Magellanic Cloud Pulsars . . . . . . . . . . . . . . . . . . . . . . . . 47

3.3 Simulated SMC/LMC Survey Results . . . . . . . . . . . . . . . . 49

4.1 Barycentered Time Samples . . . . . . . . . . . . . . . 61

5.1 SMC Survey Pointing Information . . . . . . . . . . . . . . . . 85

5.2 Parameters of Known SMC Pulsars . . . . . . . . . . . . . . . . . . . 94 


\section{List of Figures}

1.1 Lighthouse Model of Pulsar Emission . . . . . . . . . . . . . . . . . . 2

1.2 The Pulsar $P-\dot{P}$ Diagram . . . . . . . . . . . . . . . 5

1.3 Evolution on the $P-\dot{P}$ Diagram . . . . . . . . . . . . . . . . 10

1.4 Plan View of Galactic Pulsars . . . . . . . . . . . . . . . . . . 12

1.5 Pulse Scattering . . . . . . . . . . . . . . . . . . . . . 13

1.6 Pulsar Luminosity Distribution . . . . . . . . . . . . . 16

2.1 Alignment Angle Decay . . . . . . . . . . . . . . . . . . . . . 22

2.2 Evolution of Simulated Models . . . . . . . . . . . . . . . . . . 32

2.3 Histograms of Simulated vs. Observed Data . . . . . . . . . . . . 34

$2.4 P-\dot{P}$ Diagrams of Selected Models . . . . . . . . . . . . . . 35

3.1 Number of Normal Pulsars Generated . . . . . . . . . . . . . . . . . 50

3.2 Number of MSPs Generated . . . . . . . . . . . . . . . . 52

4.1 Pulsar Search Flow Diagram . . . . . . . . . . . . . . . 60

$4.2 \mathrm{~S} / \mathrm{N}$ as a Function of DM . . . . . . . . . . . . . . 64

4.3 Periodicity Search Candidate - Good . . . . . . . . . . . . . 69

4.4 Periodicity Search Candidate - Bad . . . . . . . . . . . . . 70

4.5 Periodicity Search Candidate - RFI . . . . . . . . . . . . 71

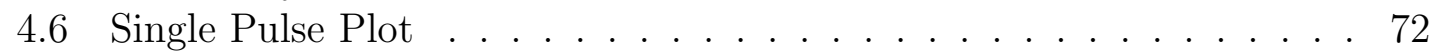

4.7 Single Pulse Plot, Cleaned . . . . . . . . . . . . . . . . . . . . . 74

4.8 Range of Detectable Binary Pulsars . . . . . . . . . . . . . . 78

5.1 Sensitivity of SMC Searches . . . . . . . . . . . . . . . 80

5.2 SMC Survey Region . . . . . . . . . . . . . . . . . . . . 82

5.3 Redetected Pulsar J0113-7220. . . . . . . . . . . . . . . . . . 86

5.4 Redetected Pulsar J0131-7310. . . . . . . . . . . . . . . 87

5.5 Redetected Pulsar J0057-7201. . . . . . . . . . . . . . . . 88

5.6 MSP Candidate SMC J0042-7300 . . . . . . . . . . . . . . . . . . . 89

5.7 MSP Candidate SMC J0045-7300 . . . . . . . . . . . . . . . . . . . 90

5.8 MSP Candidate SMC J0111-7350 . . . . . . . . . . . . . . . . . . . . . . . . 91

5.9 Bayesian Analysis of Bursting Sources . . . . . . . . . . . . . 97

A.1 PSRPOP Website Output . . . . . . . . . . . . . 108

B.1 Chandra X-ray Image of SNR G1.9+0.3. . . . . . . . . . . . 111 


\section{List of Abbreviations and Symbols}

\begin{tabular}{|c|c|}
\hline$\nu$ & Rotational Frequency \\
\hline$\rho$ & Angular Beam Radius \\
\hline$\phi$ & Pulse Phase \\
\hline$\chi$ & Magnetic Inclination Angle \\
\hline$\Omega$ & Angular Frequency \\
\hline ATNF & Australia Telescope National Facility \\
\hline$B$ & Magnetic Field \\
\hline BPSR & Berkeley-Parkes-Swinburne Data Recorder \\
\hline$b$ & Galactic Latitude \\
\hline$c$ & Speed of Light \\
\hline DFT & Discrete Fourier Transform \\
\hline $\mathrm{DM}$ & Dispersion Measure \\
\hline$d$ & Distance to the Pulsar \\
\hline FFT & Fast Fourier Transform \\
\hline FT & Fourier Transform \\
\hline$f$ & Observational Frequency \\
\hline GUPPI & Green Bank Ultimate Pulsar Processing Instrument \\
\hline$I$ & Moment of Inertia \\
\hline IRAS & Infrared Astronomical Satellite \\
\hline ISM & Interstellar Medium \\
\hline$i$ & Orbital Inclination Angle \\
\hline Jy & Jansky \\
\hline KS & Kolmogorov-Smirnoff \\
\hline $\mathrm{kpc}$ & Kiloparsec \\
\hline$L$ & Luminosity \\
\hline $\mathrm{LMC}$ & Large Magellanic Cloud \\
\hline$l$ & Galactic Longitude \\
\hline$M_{\odot}$ & Mass of the Sun \\
\hline MJD & Modified Julian Day \\
\hline MSP & Millisecond Pulsar \\
\hline$n$ & Braking Index \\
\hline$n_{e}$ & Electron Density \\
\hline$P$ & Rotational Period \\
\hline$\dot{P}$ & Rotational Period Derivative \\
\hline PDF & Probability Density Function \\
\hline PSR & Pulsating Source of Radio Emission \\
\hline $\mathrm{R}$ & Neutron Star Radius \\
\hline RFI & Radio Frequency Interference \\
\hline$S$ & Flux Density \\
\hline $\mathrm{S} / \mathrm{N}$ & Signal-to-Noise Ratio \\
\hline SKA & Square Kilometre Array \\
\hline $\mathrm{SMC}$ & Small Magellanic Cloud \\
\hline SNR & Supernova Remnant \\
\hline
\end{tabular}




\section{Chapter 1}

\section{Introduction}

\subsection{A Brief History of Pulsars}

Pulsars were accidentally discovered by Jocelyn Bell in 1967 (Hewish et al., 1968). In a search for quasars in the radio portion of the electromagnetic spectrum (centered at a frequency of $81.5 \mathrm{MHz}$ with a bandwidth of $1 \mathrm{MHz}$ ), a periodic signal, occurring every 1.337 seconds, was detected. At first, they were dismissed as radio interference generated somewhere on the Earth. However, when the signals disappeared on a daily basis and then reappeared 4 minutes earlier the next day, it was determined that they must be of celestial origin.

The simplest model to explain the dynamics of a pulsar is the rotating neutron star model. Born in supernovae, neutron stars (Baade \& Zwicky, 1934) with very high magnetic fields $\left(>10^{8} \mathrm{G}\right)$ and very short $(<12 \mathrm{~s})$ spin periods are identified as pulsars. Because the spin and magnetic field axes are misaligned, pulses of radiation emitted at the spin frequency are observed, similar to how a lighthouse shines a beam of light (see Figure 1.1).

Pulsars are named after their position in the sky. The pulsar's right ascension, in hours and minutes, and declination, in degrees and minutes, determine the pulsar's name. Bell's pulsar, for example, has the name J1921+1919, meaning that its $\mathrm{RA}$ is $19 \mathrm{~h} 21 \mathrm{~m}$ and its declination is $+19^{\circ} 19^{\prime}$. Due to the precession and nutation 


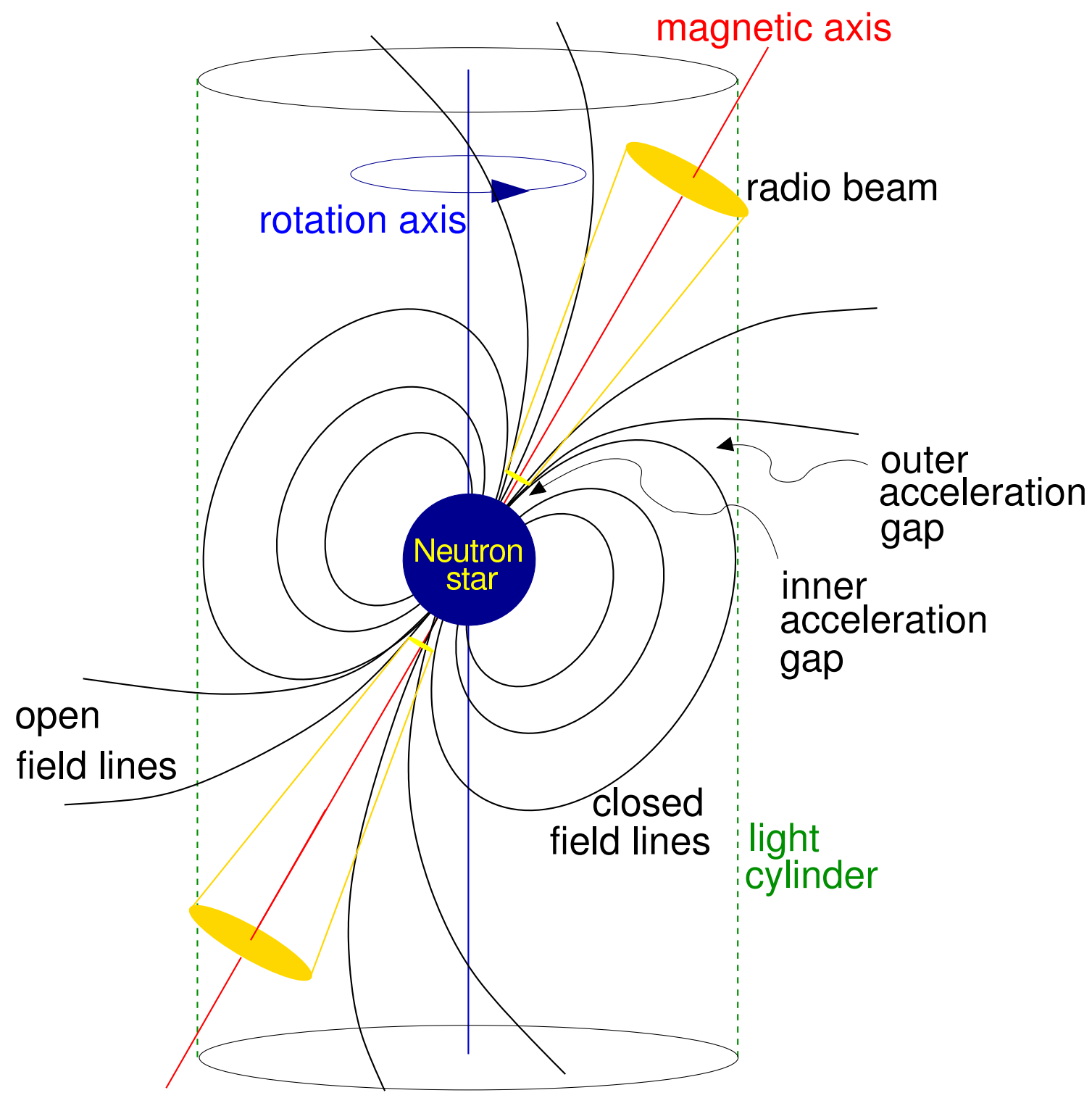

Figure 1.1: The misalignment of the rotation and magnetic field axes cause pulses of emission to be observed each time the radio beam passes through the line of sight of the observer. The radius of the light cylinder is defined by the point at which magnetic field lines co-rotating with the pulsar are moving at the speed of light. From this, closed field lines are magnetic field lines that close within the light cylinder radius, while open field lines are ones that do not. For more details regarding this model, see Lorimer \& Kramer (2005). 
of Earth's spin axis, apparent celestial positions are always changing. Therefore, these positions refer to one of two different epochs, or reference dates. A letter "B", short for Besselian, in front of the position means that January 1, 1950 was used as the epoch reference position, while a letter "J", for Julian, signifies the position as of January 1, 2000.

These pulsars can be broken into two main subcategories: millisecond pulsars (MSPs) and normal pulsars. Normal pulsars typically have higher magnetic fields $\left(\sim 10^{12} \mathrm{G}\right)$ and spin periods from $20 \mathrm{~ms}$ to $10 \mathrm{~s}$. These pulsars are usually born in supernovae, slow down over time, and eventually cease their radio emission. MSPs have slightly lower magnetic fields $\left(10^{8}-10^{9} \mathrm{G}\right)$ and spin periods less than $20 \mathrm{~ms}$. They are often found in binary systems with white dwarfs. They attain their high spin rates and low magnetic fields due to the accretion of matter from its companion star, in a process known as recycling (Bhattacharya \& van den Heuvel, 1991). An additional class of neutron stars also exhibits similar behavior to those of pulsars. Magnetars (Duncan \& Thompson, 1992), or neutron stars with very high magnetic fields $\left(\sim 10^{15} \mathrm{G}\right)$, are not as well understood as regular pulsars, mostly because there are only 16 of them known today ${ }^{1}$. All told, there are currently about 1800 known pulsars, cataloged in the Australia Telescope National Facility (ATNF) Pulsar Database ${ }^{2}$ (Manchester et al., 2005).

\footnotetext{
${ }^{1}$ See the McGill Online Catalog at http://www.physics.mcgill.ca/ pulsar/magnetar/main.html. ${ }^{2}$ Available at http://www.atnf.csiro.au/research/pulsar/psrcat.
} 


\subsection{Pulsars Unfurled}

There are many properties of pulsars that can be observed. For a detailed description, see Lorimer \& Kramer (2005). A few of the most relevant ones for this thesis are discussed in the following sections.

\subsubsection{Rotation Rates}

Since pulsars rotate with a spin period $P$, an angular frequency of rotation, $\Omega$, can be measured. As will be discussed later, pulsars slow down over time, which means that $\Omega$ will decrease. Pulsar timing observations measure the phase of the pulse, $\phi(t)$, which can be modeled, in the simplest case, via a Taylor expansion of pulse phase at time $\mathrm{t}$ as follows:

$$
\phi(t)=\phi\left(t_{0}\right)+\Omega_{0}\left(t-t_{0}\right)+\frac{1}{2} \dot{\Omega}\left(t-t_{0}\right)^{2}+\ldots,
$$

where $t_{0}$ is the initial time, $\Omega_{0}$ is the initial frequency measured at $t=t_{0}$, and $\dot{\Omega}$ is the time derivative of $\Omega$. Frequently, these rotation rates are presented as the spin period and the period derivative $(\dot{P})$, where $\Omega=2 \pi / P$ and $\dot{\Omega}=-2 \pi \dot{P} / P^{2}$. Values are between $1.39 \mathrm{~ms}$ and $11.8 \mathrm{~s}$ for $P$ and $10^{-21}$ to $10^{-10} \mathrm{~s} / \mathrm{s}$ for $\dot{P}$.

The standard method of classifying pulsars is by plotting $P$ and $\dot{P}$ on what is known as the $P-\dot{P}$ diagram (see Figure 1.2). The normal pulsar population takes up the majority of the diagram, the MSPs can be found in the lower left corner, and magnetars are found in the upper right corner. Nearly $80 \%$ of all MSPs are found 


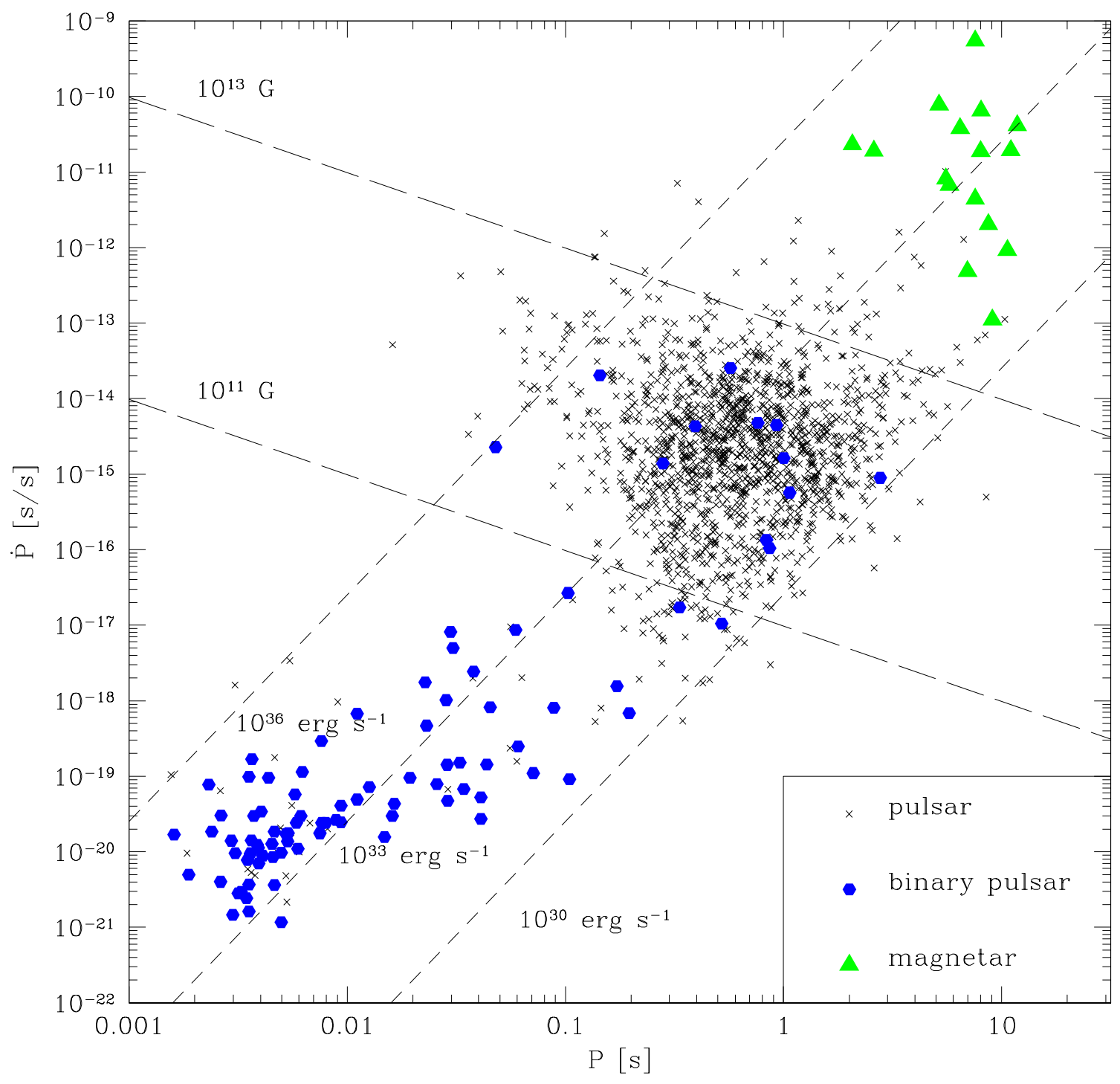

Figure 1.2: A collection of known pulsars plotted on the $P-\dot{P}$ diagram. The period is plotted with units of seconds, while $\dot{P}$ has units of s/s. The blue filled circles represent pulsars in binary systems, while the green triangles are magnetars. Short dotted lines represent lines of constant age, medium dashed lines show constant $\dot{E}$, and long dashed lines are lines of constant magnetic field. 
in binary star systems, however, less than $1 \%$ of all normal pulsars have a binary companion (Lorimer \& Kramer, 2005).

\subsubsection{Rotational Kinetic Energy}

Pulsars have a substantial amount of rotational kinetic energy $\left(E_{\mathrm{rot}}=\frac{1}{2} I \Omega^{2}\right)$ due to their large moment of inertia and their very fast rotation rate, $\Omega$. If the pulsar is treated as a uniform sphere, then $I=\frac{2}{5} M R^{2}$, where $M$ is the mass of the pulsar and $R$ is its radius. Assuming canonical values of $M=1.4 \mathrm{M}_{\odot}$ (Thorsett \& Chakrabarty, 1999) and $R=10 \mathrm{~km}$ (Lattimer \& Prakash, 2001), the moment of inertia can be estimated as $10^{45} \mathrm{~g} \mathrm{~cm}^{2}$, and thus $E_{\text {rot }}$ of a $1 \mathrm{~s}$ pulsar would be nearly $10^{46}$ ergs.

As pulsars slow down, they lose some of this rotational kinetic energy:

$$
\dot{E}=-\frac{d}{d t}\left(E_{\text {rot }}\right)=-I \Omega \dot{\Omega}
$$

If the spin frequencies are converted to $P$ and $\dot{P}$ and the above value of $I$ is used, this loss of energy can be represented as:

$$
\dot{E}=3.95 \times 10^{31} \mathrm{erg} \mathrm{s}^{-1}\left(\frac{\dot{P}}{10^{-15}}\right)\left(\frac{P}{1 \mathrm{~s}}\right)^{-3}
$$

As seen in Figure 1.2, most of the known pulsars have an $\dot{E}$ between $10^{30}$ and $10^{36}$ $\mathrm{erg} \mathrm{s}^{-1}$. The Sun has a luminosity of $3.83 \times 10^{33} \mathrm{erg} \mathrm{s}^{-1}$, meaning that the Sun's rate of energy loss is similar to that of a typical pulsar. 
The lower $\dot{E}$ value of $10^{30} \mathrm{erg} \mathrm{s}^{-1}$ is very close to what is known as the pulsar death line (Chen \& Ruderman, 1993). Very few pulsars are found on the $P-\dot{P}$ diagram below this line, and, theoretically, this is the point at which a pulsar ceases to have radio emission. The emission process of pulsars is not entirely understood. Pair production (creation of an electron and a positron from a photon) caused by a rotating magnetic field is considered to be a possible means of generating radio emission. The pulsar's "death" occurs when it no longer spins fast enough to rotate the magnetic field at a speed in which it is possible for pair production to occur.

\subsubsection{Magnetic Field}

The pulsar's magnetic field strength, $B$, is very large when compared to the Earth or Sun. If the loss of rotational kinetic energy is equated to the power radiated from a magnetic dipole, Ostriker \& Gunn (1969) found that the magnetic field at the surface of the pulsar is

$$
B_{s}=\sqrt{\frac{3 c^{3}}{8 \pi^{2}} \frac{I}{R^{6} \sin ^{2} \chi} P \dot{P}}
$$

where $\chi$ is the angle between the spin axis and the magnetic dipole axis, and $c$ is the speed of light. Assuming $I=10^{45} \mathrm{~g} \mathrm{~cm}^{2}$ and setting $\chi=90^{\circ}$, Manchester \& Taylor (1977) obtain a surface magnetic field of

$$
B=3.2 \times 10^{19} \mathrm{G} \sqrt{P \dot{P}},
$$


where $P$ is measured in seconds and $\dot{P}$ has units of s/s. Again, Figure 1.2 shows that nearly all of the normal pulsars have a magnetic field between $10^{11} \mathrm{G}$ and $10^{13} \mathrm{G}$. MSPs have considerably weaker magnetic fields, while the magnetars have much stronger fields.

\subsubsection{Braking Index and Spin-down}

The rotational frequency, $\nu$, and frequency derivative, $\dot{\nu}$, can be related by a more general model for the loss of rotational kinetic energy as follows:

$$
\dot{\nu}=-\mathrm{K} \nu^{n}
$$

Here, $n$ is defined to be the braking index of the pulsar and $\mathrm{K}$ is a constant (Lorimer \& Kramer, 2005). The magnetic dipole model discussed above leads to a value of 3 for $n$. If Equation 1.6 is differentiated, the braking index can be represented by

$$
n=\frac{\nu \ddot{\nu}}{\nu^{2}}
$$

Typically, the braking index is calculated by timing the pulsar (i.e. measuring $\nu, \dot{\nu}$, and $\ddot{\nu})$ and the six known pulsars with measured braking indices have values ranging from 1.4 to 2.84 (see Livingstone et al. (2006) and Weltevrede et al. (2010)). The major challenge of measuring a braking index is obtaining $\ddot{\nu}$. Measuring the small deviations of $\ddot{\nu}$ require very spin high frequencies which explains why only young, rapidly spinning pulsars have measured braking indices. 


\subsubsection{Evolution on the $P-\dot{P}$ Diagram}

The braking index dictates how quickly the pulsar moves across the $P-\dot{P}$ diagram. As seen in Figure 1.3, the path a pulsar takes across the diagram depends greatly on the braking index of the pulsar. A pulsar with a lower braking index will generally attain a longer period before reaching the death line.

\subsubsection{Dispersion Measure}

The interstellar medium (ISM) significantly affects pulsar signals. Intuitively, the more material through which the signal must propagate, the slower the signal will arrive. Also affecting this is the fact that a larger distance generally means more material through which to travel. This second effect can be represented by a quantity known as the Dispersion Measure (DM). The DM can be defined as the integrated column density of free electrons along the line of sight to the pulsar:

$$
\mathrm{DM}=\int_{0}^{d} n_{e} \mathrm{~d} l
$$

where $d$ is the distance to the pulsar and $n_{e}$ is the electron density along the line of sight to the pulsar. The DM is measured in units of $\mathrm{pc} \mathrm{cm}^{-3}$ and can be calculated by measuring the pulse arrival times at two different frequencies. This time delay, $\Delta t$, can be represented as

$$
\left.\Delta t=4.15 \times 10^{6} \mathrm{~ms} \times\left[\left(\frac{f_{1}}{\mathrm{MHz}}\right)^{-2}-\left(\frac{f_{2}}{\mathrm{MHz}}\right)^{-2}\right] \times\left(\frac{\mathrm{DM}}{\mathrm{pc} \mathrm{cm}}\right)^{-3}\right)
$$




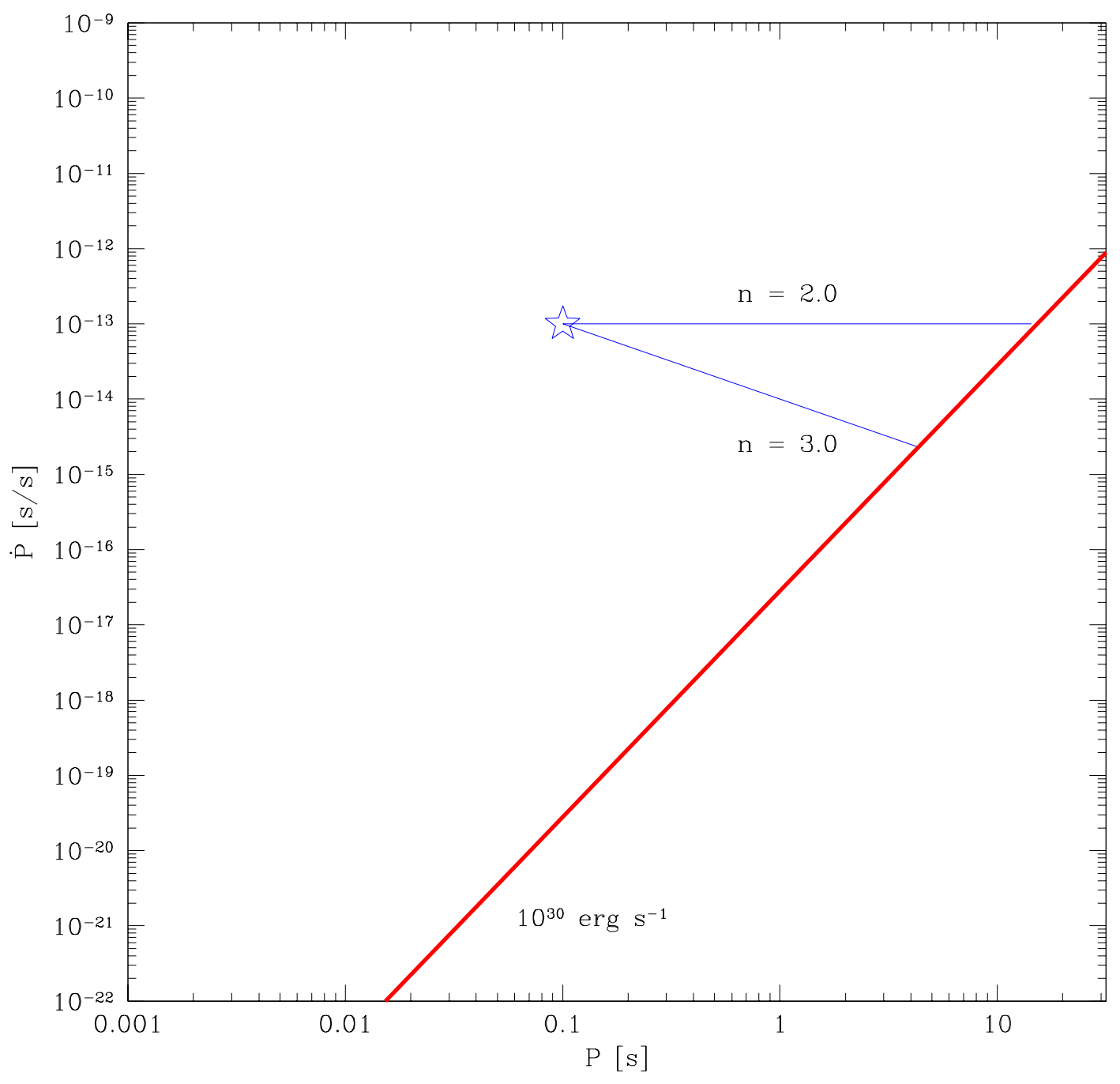

Figure 1.3: Two evolutionary tracks across the $P-\dot{P}$ diagram are shown for a pulsar with an initial period of $100 \mathrm{~ms}$ and $\dot{P}$ of $10^{-13} \mathrm{~s} / \mathrm{s}$ and braking indices of 2.0 and 3.0. A lower braking index results in a greater "distance" traveled and allows the pulsar to reach a longer period before ceasing radio emission. The death line is very closely represented by a line of $\dot{E}=10^{30} \mathrm{erg} \mathrm{s}^{-1}$. 
where $f_{1}$ and $f_{2}$ are the lower and upper frequencies. If the DM is known, then Equation 1.8 can be numerically integrated to determine the distance to the pulsar. Figure 1.4 shows how the electron density varies throughout the Galaxy as well as how the currently known pulsars are positioned. As can be seen in Figure 1.4, pulsars located near the center of the Galaxy are usually found by high frequency surveys rather than low frequency ones. This is because the DM towards the center of the Galaxy is usually quite high. The high DM, coupled with low frequency observations, increases the time delay found in Equation 1.9 more than if a high frequency observation is used, which makes the pulsar harder to detect.

\subsubsection{Scattering}

In addition to pulse dispersion, scattering also affects a pulsar's detectability. As a pulse travels through the interstellar medium, many of the waves become scattered and therefore will have a different path length from the pulsar to the detector. The delayed arrival times of these scattered waves result in the broadening of the final pulse shape, many times with a scattering tail at the end of the pulse (see Figure 1.5).

Generally, pulsars with high DMs are more likely to be affected by scattering. In addition, the scattering time, $\tau_{\mathrm{s}}$, is proportional to $f^{-4}$, meaning that scattering is more severe at very low frequencies (Lorimer \& Kramer, 2005). 


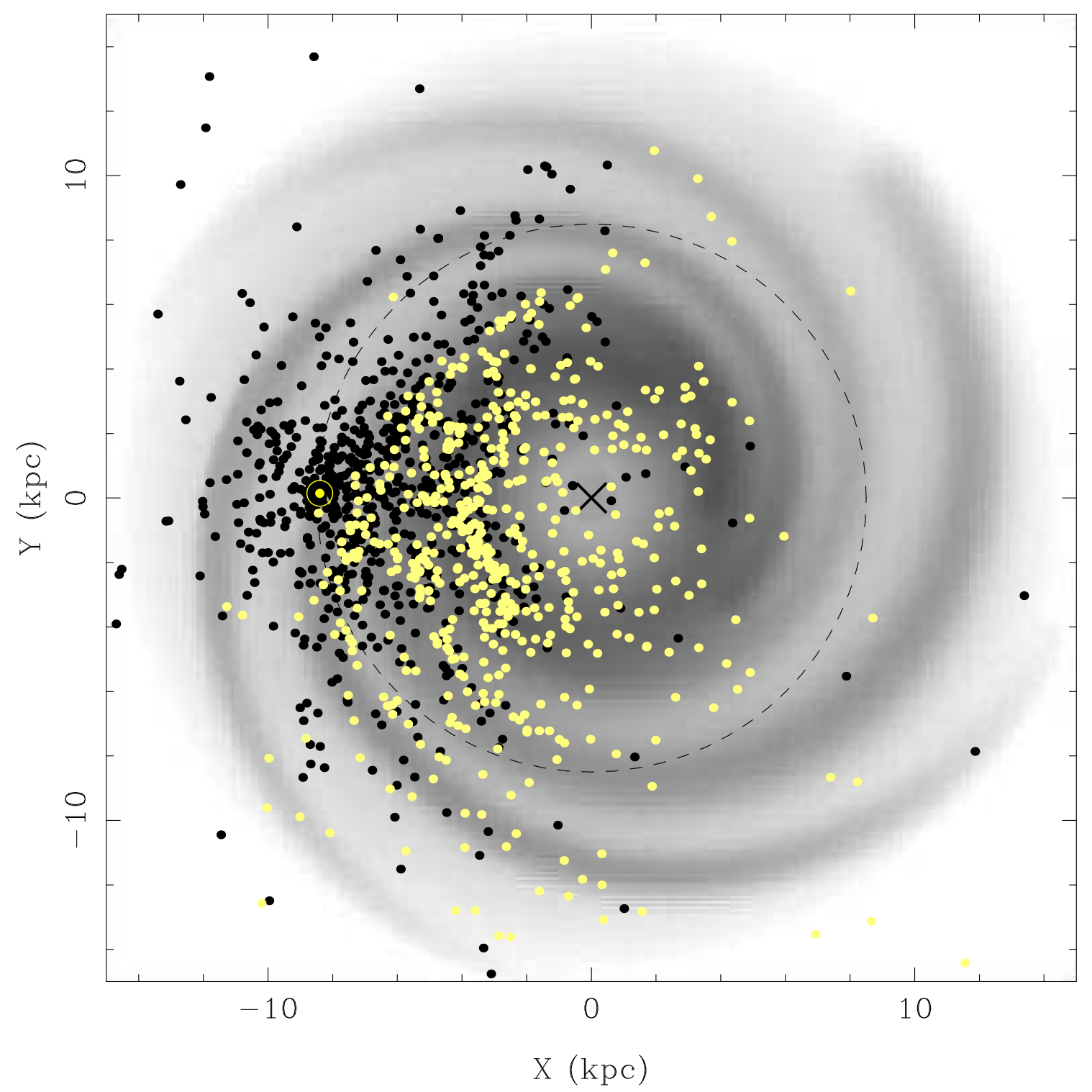

Figure 1.4: An X-Y plot of all pulsars in the plane of the Galaxy, with the Sun circled and an $\mathrm{X}$ marking the center of the Galaxy, overlaid on the distribution of electrons throughout the Galaxy (Lorimer \& Kramer, 2005). Dark points are pulsars that were discovered in low frequency $(430 \mathrm{MHz})$ surveys, while the lighter points are pulsars found in high frequency $(1400 \mathrm{MHz})$ surveys. 


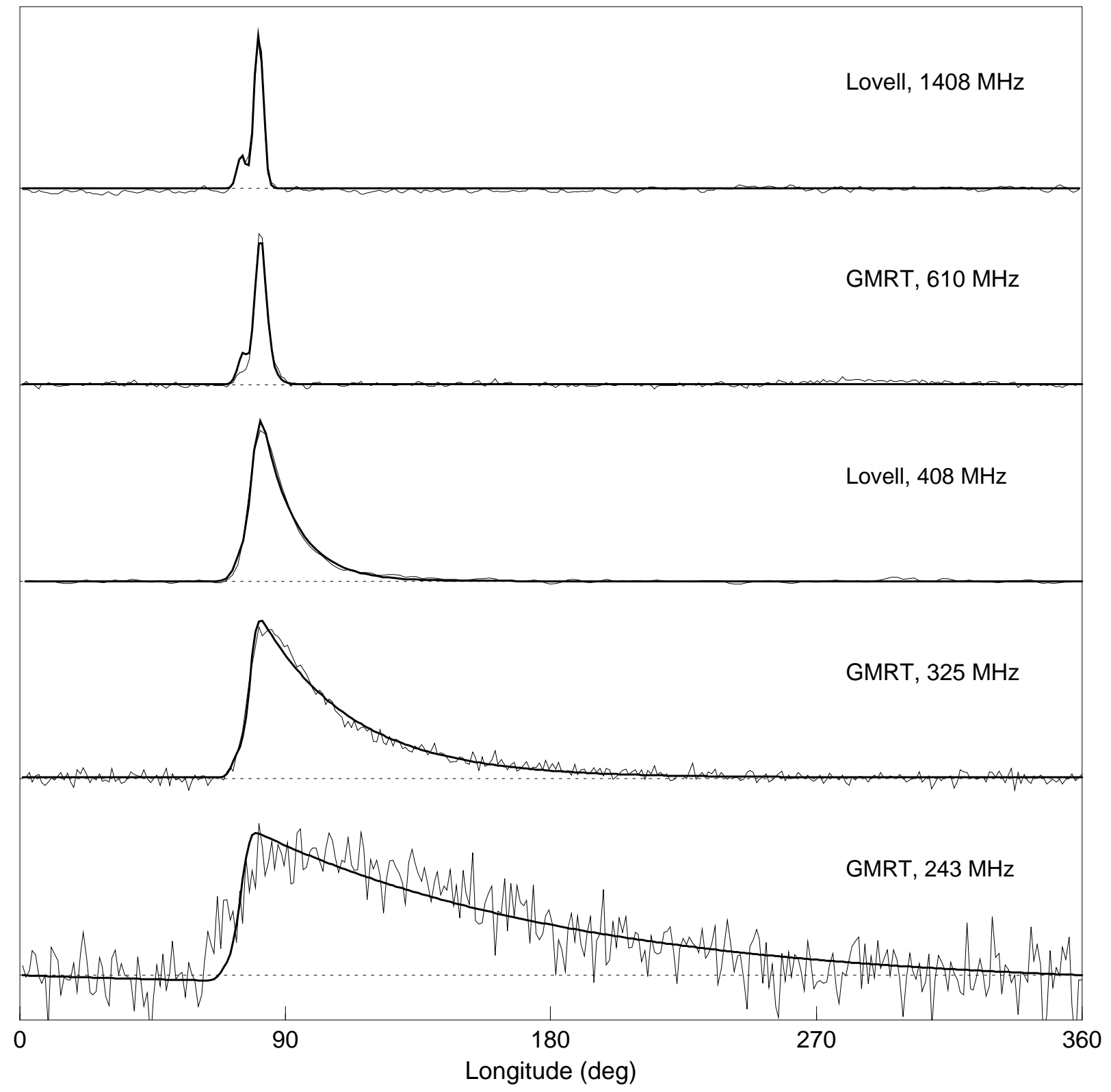

Figure 1.5: Observations at low frequencies show an exponential tail (shown by the solid lines) in PSR B1831-03 due to the effects of scattering (Löhmer et al., 2004). 


\subsubsection{Luminosity and Flux Density}

One inherent property of a pulsar that can be indirectly observed is its radio luminosity, $L$, or brightness, which is related to the energy loss of the pulsar. By measuring the flux density, $S$, with a radio telescope, the luminosity can then be calculated by

$$
L=S d^{2}
$$

where $d$ is the distance to the pulsar. As seen by Equation 1.10, the relationship between a pulsar's luminosity and the observed flux obeys the inverse square law. In general, the luminosity is defined as $L=\omega S d^{2}$, where $\omega=\int_{\text {beam }} \mathrm{d} \Omega$ is the solid angle covered by the emission of the pulsar's radio beam, with $\omega=4 \pi$ for isotropic radiation and $\omega=0$ for no emission. Since the beam size of a pulsar's radio emission varies and is largely unknown, the factor of $\omega$ is usually omitted and this "pseudoluminosity" creates a standard reference point so that all pulsars can be easily compared to each other, regardless of beam size.

Flux densities measured at $1400 \mathrm{MHz}$ range from $3.4 \mu \mathrm{Jy}$ to $1.1 \mathrm{Jy}$, where the unit of flux density is the Jansky (Jy), and $1 \mathrm{Jy}=10^{-26} \mathrm{~W} \mathrm{~m}^{-2} \mathrm{~Hz}^{-1}$. The mean of the $\log$ of the luminosities (measured at a center frequency of $1400 \mathrm{MHz}$ ) of all known pulsars is 1.21 , which corresponds to a luminosity of $16.33 \mathrm{mJy} \mathrm{kpc}{ }^{2}$, based on pulsars found in the ATNF Pulsar Database. A statistical study of the distances to and fluxes of all pulsars that have not crossed the death line (and hence still have radio emission) has shown that pulsar luminosities are best represented by a $\operatorname{lognormal}$ distribution with mean $<\log L>=-1.1$ (equivalent to a luminosity 
of $0.07 \mathrm{mJy} \mathrm{kpc}^{2}$ ) for the normal pulsar population (Faucher-Giguère \& Kaspi, 2006). As telescopes become more sensitive to pulsars with lower luminosities, the average value of $16.33 \mathrm{mJy} \mathrm{kpc}^{2}$ is expected to approach the theoretical value of $0.07 \mathrm{mJy} \mathrm{kpc}{ }^{2}$. Figure 1.6 shows a histogram of the observed $1400 \mathrm{MHz}$ luminosities plotted along with the mean luminosity distribution found by Faucher-Giguère \& Kaspi (2006).

\subsection{Outline of Dissertation}

The goals of this dissertation are to produce a working model of the normal and MSP pulsar populations via computer simulations, predict the results of future pulsar surveys, and present the results of one of these new pulsar surveys. With the help of theoretical models relating to many of the previously mentioned pulsar parameters, these computer simulations allow for the studying of pulsars in ways that are otherwise unfeasible. The final survey for pulsars in the Small Magellanic Cloud successfully ties together the simulated and predicted models with the actual search results.

Chapter 2 shows the results of computer simulations of model pulsar populations. In these simulations, the various pulsar parameters mentioned above are manipulated using Monte Carlo simulations to produce a sample distribution of pulsars that mirrors the observed pulsar population. The code used for these simulations is publically available (see Appendix A).

Chapter 3 takes this code and applies it to specific surveys of the Large and 


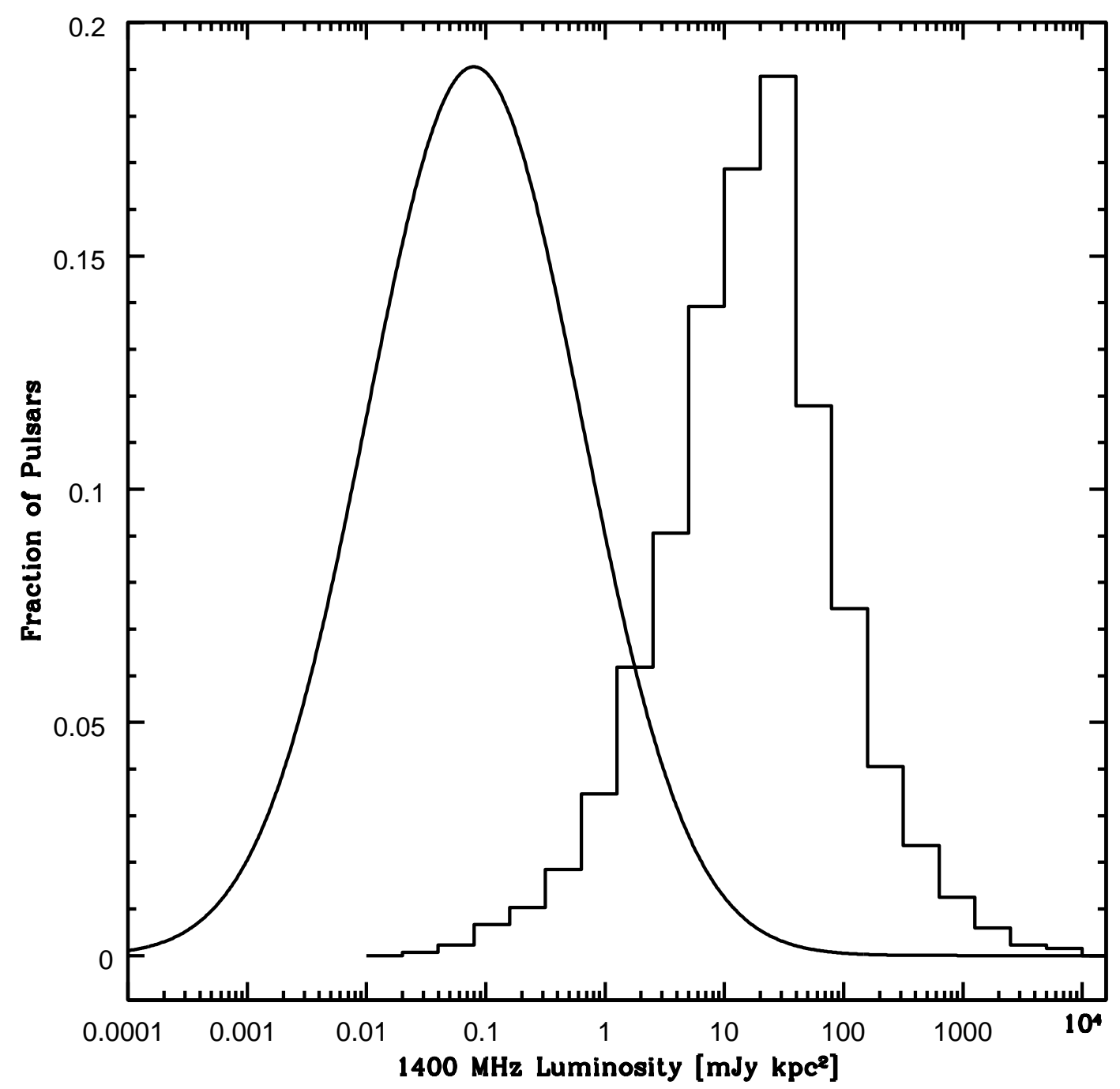

Figure 1.6: A histogram of the luminosities of known pulsars is shown along with the normal distribution of luminosities (solid line) obtained from Faucher-Giguère \& Kaspi (2006). The histogram has mean $<\log L>=1.21\left(L=16.33 \mathrm{mJy} \mathrm{kpc}{ }^{2}\right)$, and the normal distribution is represented by a lognormal distribution with mean

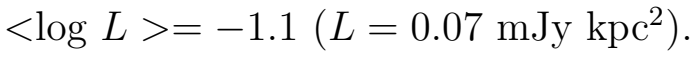


Small Magellanic Clouds (LMC and SMC). Here, past surveys were modeled and an estimated number of normal pulsars was calculated for both the LMC and SMC. After reproducing the results for the normal pulsars, the code was used to calculate an upper limit on the number of MSPs that could be present. The results from those simulations led to a survey of the SMC that was performed with the Parkes Radio Telescope in 2009.

Chapter 4 introduces pulsar searching and search techniques and provides motivation of why pulsars should be the focus of radio surveys. The basic search method, including limitations of various instruments, will be discussed. Also, various types of searches, optimized for the detection of period sources, single pulses, and pulsars in binary systems will be examined in detail.

Chapter 5 presents the results of a radio survey of the SMC. This survey was performed with the goals of detecting the first MSP in either the LMC or SMC and to find giant pulse emitting sources. Observations, data processing, and other areas involved with the survey are discussed.

The main conclusions from this work are presented in Chapter 6. Finally, there are two appendices included. Appendix A shows the features of a website that was designed to model previous and future surveys and compare various pulsar populations. Appendix B presents the results of a search that was performed with the Green Bank Telescope of the supernova remnant G1.9+0.3 with the goal of detecting the youngest pulsar in the Galaxy. 


\section{Chapter 2}

\section{Isolated Pulsar Spin Evolution on the $P-\dot{P}$ Diagram}

This chapter was originally published as a refereed paper in the Monthly Notices of the Royal Astronomical Society (MNRAS) by J. P. Ridley and D. R. Lorimer in April of 2010. Minor wording changes from the original accepted paper have been made where appropriate.

\subsection{Introduction}

The statistical properties of the radio pulsar population provide valuable constraints on the birth properties, evolution and radio lifetimes of neutron stars and have been the subject of many studies over the years (see, e.g., Gunn \& Ostriker, 1970; Taylor \& Manchester, 1977; Lyne et al., 1985; Narayan \& Ostriker, 1990; Bhattacharya et al., 1992; Johnston, 1994; Lorimer et al., 1997; Hartman et al., 1997; Arzoumanian et al., 2002; Gonthier et al., 2002; Faucher-Giguère \& Kaspi, 2006). A key diagram to reproduce is the logarithmic plot of pulsar periods, $P$, and their rates of slowdown, $\dot{P}$, usually referred to as the $P-\dot{P}$ diagram. The many factors that affect the distribution of pulsars on the $P-\dot{P}$ diagram include the evolution of

pulsars' spin-down, luminosity, age, magnetic field, beaming, and the angle between the magnetic field and the rotation axis. While each parameter individually affects how pulsars evolve across the diagram, covariances between multiple parameters 
also influence the results.

The goal of this work is to compare two pulsar spin-down models using Monte Carlo simulations of the Galactic young pulsar population. The first model that is considered treats the pulsar as a rotating magnetic dipole from which there is a simple relationship between the braking torque and the magnetic field (Gold, 1968; Pacini, 1968). This model has been used extensively in pulsar population studies in the past (see, e.g. Lyne et al., 1985; Narayan \& Ostriker, 1990; Bhattacharya et al., 1992) and has most recently been implemented by Faucher-Giguère \& Kaspi (2006), hereafter FK06. Here, a direct comparison of their results with new computer simulations is carried out.

The other model considered, by Contopoulos \& Spitkovsky (2006), hereafter CS06, treats the pulsar as a combination of a misaligned magnetic dipole rotating in vacuum and an aligned magnetic dipole rotating in an atmosphere with ideal magnetohydrodynamic conditions. In addition to implementing these two models with parameters as described in detail in FK06 and CS06, the effects of changing one or more of the assumptions made by these authors are investigated in an attempt to obtain a more accurate interpretation of the relationships between these parameters and the resulting $P-\dot{P}$ diagram.

As described in Section 2.2, a computer simulation package has been developed which implements the above models and allows the distribution of pulsars in the Galaxy to be simulated and the individual properties of each one to be changed. By "observing" this population with accurate models of completed pulsar surveys (see Section 2.3), a simulated sample of pulsars can be created and its properties 
can be compared to those of the real observed sample. In Section 2.4, the method for comparing two population samples is described. In Section 2.5, the main results are presented and discussed. The main conclusions are summarized in Section 2.6. To facilitate comparison with other studies, and provide a tool for the community, a major feature of this work is to make the simulation code freely available on a website which is discussed in Appendix A.

\subsection{Modeling the Underlying Pulsar Population}

The computer program that was developed for this work, EVOLVE, is an extension of the software package, PSRPOP, which was initially developed to study the Galactic distribution of pulsars detected in the Parkes Multibeam surveys (Lorimer et al., 2006). For that work, time-independent pulsar populations were synthesized and compared to the observed sample. The main aim of this work is to develop a time-dependent model of the population and is implemented through the EVOLVE computer program. In this section, the logical flow of the program is described, which determines first whether a model pulsar is potentially observable (i.e. is radio loud and beaming towards Earth) before computing any kinematic or spatial parameters.

The first step is to randomly assign an age, $t$, and a magnetic field, $B$, to each pulsar. The age is selected between 0 and $t_{\max }$, with $t_{\max }$ being $10^{9}$ years. As per FK06, the magnetic field is determined by a log-normal distribution, centered around a mean value, $\mu_{\log B}=12.65$, with a standard deviation $\sigma_{\log B}=0.55$. The 
initial period, $P_{0}$, is then randomly generated. Following FK06, $P_{0}$ is chosen from a Gaussian distribution centered around $\mu_{P}=300 \mathrm{~ms}$ with a standard deviation $\sigma_{P}=150 \mathrm{~ms}$.

Next, the inclination angle between the magnetic field axis and spin axis, $\chi$, is computed. In implementations of the magnetic dipole model, it is usually assumed (see, e.g., FK06) that $\chi=90^{\circ}$. However, a more realistic model would account for a distribution of $\chi$ and use this information in a self-consistent way when calculating the beaming fraction discussed below. A number of options are explored, first fixing $\chi=90^{\circ}$, and subsequently considering a random distribution of angles by selecting $\cos \chi$ from a flat distribution between 0 and 1 . The latter choice effectively orients the magnetic axis as a uniform random vector over $4 \pi$ sr. Finally, following recent empirical evidence in favor of a secular decay of $\chi$ with time (Weltevrede \& Johnston, 2008, hereafter WJ08), an exponentially decaying model is also considered, defined as

$$
\sin \chi=\sin \chi_{0} \exp \left(-t / t_{d}\right)
$$

where $\chi_{0}$ is the initial angle (which again is chosen by selecting $\cos \chi_{0}$ from a $0-1$ flat distribution), and $t_{d}$ is the $1 / e$ decay timescale. Note that this decay law is chosen over the one considered by WJ08 for mathematical and computational convenience. As shown in Figure 2.1, the two dependencies give similar results. The braking index, $n$, is then assigned. In general, $n$ is related to $\nu$, the frequency of rotation, 


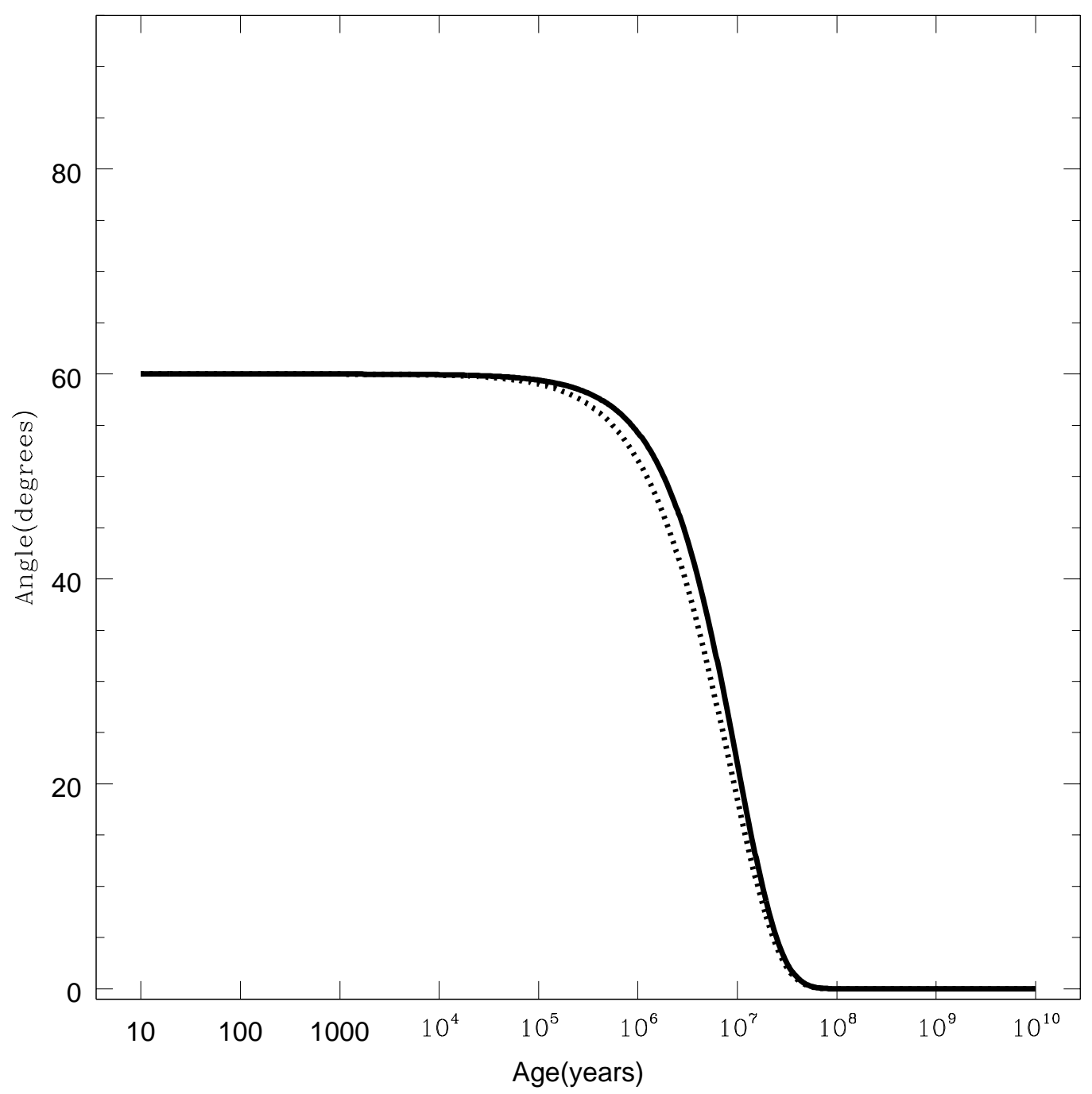

Figure 2.1: The time dependence of the alignment angle shown for Equation 2.1 (dotted curve) and the model used in WJ08 (solid curve). A reference of $60^{\circ}$ is used as roughly half of all angles will be above and half will be below that value for a given random distribution of angles. 
$\nu=1 / P$, and its time derivative

$$
\dot{\nu}=-K \nu^{n},
$$

where $K$ is a constant that depends on the pulsar's moment of inertia and radius (see, e.g. Lorimer \& Kramer, 2005). A single index can be used for all pulsars, or one can randomly be assigned to each pulsar. Specific choices depend on the spin-down model under consideration which are described below.

Each spin-down model yields a time-dependent equation for the pulsar's period and period derivative. The pulsar's period evolves in time depending on the spin-down model, the magnetic field, the braking index, and the alignment angle. Considering first the magnetic dipole model, the period dependence can be found by solving

$$
P^{n-2} \dot{P}=k B^{2} \sin ^{2} \chi(t),
$$

where $\sin \chi(t)$ is defined by Equation 2.1. The constant

$$
k=\frac{8 \pi^{2} R^{6}}{3 I c^{3}},
$$

where $R$ is the radius of the neutron star, assumed to be $10 \mathrm{~km}$, and $I$ is the moment of inertia, assumed to be $10^{38} \mathrm{~kg} \mathrm{~m}^{2}$. For this choice of parameters, $k$ takes the numerical value $9.768 \times 10^{-40}, B$ is in units of Gauss while $P, P_{0}$ and $t$ are in seconds.

The implementation of this model in FK06 assumes $n=3$ and $\chi=90^{\circ}$. In 
this case, the period dependence

$$
P(t)=\sqrt{P_{0}^{2}+2 k B^{2} t} .
$$

More generally, for any braking index other than $n=1$, and a constant value of $\chi$, it is found that

$$
P(t)=\left[P_{0}^{n-1}+(n-1) k B^{2} t \sin ^{2} \chi\right]^{\frac{1}{n-1}} .
$$

Finally, extending this result for a time dependent inclination angle of the form assumed in Equation 2.1, it is found that

$$
P(t)=\left[P_{0}^{n-1}+\frac{(n-1)}{2} t_{d} k B^{2} \sin ^{2} \chi_{0}\left(1-e^{-2 t / t_{d}}\right)\right]^{\frac{1}{n-1}} .
$$

Depending on the model under consideration, one of Equations 2.5-2.7 is used to calculate the pulsar's current period, $P$. Equation 2.3 is then used to find the corresponding value of $\dot{P}$. At this point, a simple test is made to determine if the pulsar has crossed the death line. The death line signifies when a pulsar becomes radio-quiet, and Bhattacharya et al. (1992) quantified it as the locus of points for which

$$
\frac{B}{P^{2}}=0.17 \times 10^{12} \mathrm{G} \mathrm{s}^{-2} .
$$

If the pulsar has crossed the death line, the simulation marks the pulsar as dead (i.e. radio quiet) and moves on to create the next pulsar. If the pulsar has not crossed the line, then the simulation continues. It should be noted, however, that 
a small number of pulsars (e.g. J2144-3933; Young et al., 1999) have crossed this theoretical line and are still observable at radio frequencies.

For the implementation of the CS06 model, the technique is slightly different. The time evolved period and period-derivative are calculated by integrating and then numerically solving

$$
\dot{P}=3.3 \times 10^{-16}\left(\frac{P}{P_{0}}\right)^{2-n}\left(\frac{B}{10^{12} \mathrm{G}}\right)^{2}\left(\frac{P_{0}}{1 \mathrm{~s}}\right)^{-1}\left(1-\frac{P}{P_{\text {death }}} \cos ^{2} \chi\right),
$$

where

$$
P_{\text {death }}=\left[0.81 \times\left(\frac{B}{10^{12} \mathrm{G}}\right)\left(\frac{1 \mathrm{~s}}{P_{0}}\right)\right]^{\frac{2}{n+1}} \mathrm{~s} .
$$

In this case, the pulsar is declared dead when the period becomes greater than $P_{\text {death }}$.

To account for the fact the pulsar radiation is only beamed to some fraction of $4 \pi$ sr, a variety of beaming models have been implemented in EVOLVE. The default model is from the work of Tauris \& Manchester (1998), henceforth TM98, who found an empirical relationship for the fraction of the whole sky illuminated by a pulsar:

$$
f(P)=0.09\left[\log \left(\frac{P}{1 \mathrm{~s}}\right)-1\right]^{2}+0.03
$$

Other models from Biggs (1990), Lyne \& Manchester (1988), and Narayan \& Vivekanand (1983), henceforth B90, LM88, and NV83 respectively, determine the beaming fraction from the angular beam radius

$$
\rho=\rho_{0} P^{\gamma}
$$


where the constants $\rho_{0}$ and $\gamma$ are uniquely defined in each model. Assuming that radio waves are emitted from both poles of the pulsar and that the beams are circular beams, and following TM98 leads to a beaming fraction dependence on magnetic inclination angle of

$$
f(\rho, \chi)= \begin{cases}2 \sin \chi \sin \rho & \chi>\rho, \chi+\rho<\frac{\pi}{2} \\ \cos (\chi-\rho) & \chi>\rho, \chi+\rho>\frac{\pi}{2} \\ 1-\cos (\chi+\rho) & \chi<\rho, \chi+\rho<\frac{\pi}{2} \\ 1 & \chi<\rho, \chi+\rho>\frac{\pi}{2}\end{cases}
$$

Also available is a simple period-independent beaming model that assumes $f=0.2$ for all values of $P$. To determine whether a pulsar is beaming towards us or not, the value of $f$ computed in one of the above ways is compared with a random number between 0 and 1 . Only those pulsars for which this random number is less than or equal to $f$ are deemed to be beaming towards Earth and the simulation moves on to the calculations of the final properties of the pulsar.

To describe the location of model pulsars in the Galaxy, a regular Cartesian $(x, y, z)$ coordinate system is used with the Galactic center at the origin and the position of the Sun at $(0,8.5,0) \mathrm{kpc}$, again identical to the procedure performed by FK06. Each pulsar's initial $x$ and $y$ positions on the Galactic plane are determined by randomly choosing a point in the plane of the Galaxy along the spiral arms. The procedure described by FK06 is followed to implement the spiral arm structure. For the radial distribution, the form described by Yusifov \& Küçük (2004) is used. 
Then, dispersion away from the plane is used to determine the initial $z$ position. For this, an exponential distribution with a mean $z_{0}=50 \mathrm{pc}$ is used, and then a sign is arbitrarily chosen.

To model pulsar birth velocities, the optimal model found in FK06 is used, in which the initial velocity components of each pulsar in the $x, y$ and $z$ directions are drawn from exponential distributions with a mean absolute value of $180 \mathrm{~km} \mathrm{~s}^{-1}$. Although other distributions are available within EVOLVE, they are not investigated any further here. To model the resulting evolution in the Galactic gravitational potential, the method described by Lorimer et al. (1993) is followed, where the integration in the gravitational galactic potential is done using a model described by Carlberg \& Innanen (1987). From the final position in the model galaxy, the pulsar's distance from the Sun, $d$, is computed and its expected dispersion measure (DM) and scatter-broadening timescale at $1 \mathrm{GHz}$. The latter two quantities are derived using the NE2001 model for the Galactic distribution of free electrons (Cordes \& Lazio, 2002).

The radio luminosity is calculated, following FK06, with a $P$ and $\dot{P}$ power law dependence, defined by

$$
\log L=\log L_{0}+\alpha \log P+\beta \log \left(\dot{P} / 10^{-15}\right)+\delta_{L},
$$

where $L_{0}$ is $0.18 \mathrm{mJy} \mathrm{kpc}^{2}$, and $\delta_{L}$ is randomly chosen from a normal distribution with $\sigma_{\delta_{L}}=0.8$. The program allows for $\alpha$ and $\beta$, two free parameters, to be varied to determine the best functional dependence. 
For comparison, a simple model in which the luminosity is independent of all other parameters is available in the code. Following FK06, the probability, $p(L)$, of a given luminosity is taken to be

$$
p(L) \propto \begin{cases}0 & L \in\left[0 \mathrm{mJy} \mathrm{kpc}{ }^{2}, 0.1 \mathrm{mJy} \mathrm{kpc}^{2}\right) \\ L^{-\frac{19}{15}} & L \in\left[0.1 \mathrm{mJy} \mathrm{kpc}^{2}, 2.0 \mathrm{mJy} \mathrm{kpc}^{2}\right) \\ L^{-2} & L \in\left[2.0 \mathrm{mJy} \mathrm{kpc}{ }^{2}, \infty\right) .\end{cases}
$$

\subsection{Modeling the Observed Pulsar Population}

The steps described above allow a population of synthetic pulsars that are potentially observable to be created. That is, they are deemed to be beaming towards the Earth and the spin-down models that are being investigated predict that they are radio-loud. This population is then compared with those pulsars that are potentially detectable by current surveys. Since the primary objective of this work is to reproduce and extend upon the work of FK06, the main focus is on the sample of pulsars detectable by the Parkes Multibeam (see Lorimer et al., 2006, and references therein, hereafter PMB) and Swinburne (Edwards et al., 2001, hereafter SMB) Multibeam pulsar surveys. By accurately modeling the detection thresholds of these surveys as described below, and selecting only those model pulsars that are theoretically detectable, samples of model observed pulsars can be formed. When FK06 were carrying out their work, the sample of real pulsars detected in these surveys was 1065 . In this analysis, the sample of 1135 pulsars currently cataloged will be used. All isolated pulsars not associated with a globular cluster and having 
both $\dot{P}<10^{-12}$ and $P>30 \mathrm{~ms}$ are selected as part of this sample.

To form the model observed samples, the first step is to compute the apparent flux density of each model pulsar, S. Following standard practice (see Chapter 1), geometrical factors in the inverse square law relationship are neglected and $S$ is found from the pulsar's distance, $d$, and radio luminosity, $L$ (see Equation 1.10. Note that this flux density is defined (following the definition of $L$ in this work) to be at an observing frequency of $1.4 \mathrm{GHz}$, the frequency at which the PMB and SMB surveys were carried out. For the purposes of this work, no assumptions about the pulsar spectral index distribution are necessary.

To model the detection threshold of the pulsar surveys, it is also necessary to model the pulse widths. Following FK06, it is assumed that the intrinsic pulse width, $W_{\text {int }}$, is $5 \%$ of the pulse period. The observed pulse width, $W_{\text {obs }}$, and signal-to-noise ratio, $\mathrm{S} / \mathrm{N}$, are then calculated using the method described in Lorimer et al. (2006) in their Equations 1-7. Pulse width models from recent papers (see, e.g., Smits et al., 2009) have also been considered and are available for use in the code.

All pulsars which lie inside the sky boundaries covered by the two surveys, have $\mathrm{S} / \mathrm{N}>9$, and where $W_{\mathrm{obs}}<P$ are deemed detectable and saved for subsequent analysis, as described in the following sections. This simulation proceeds until 1135 pulsars are detected, to match the combined sample found in the PMB and SMB surveys. 
Table 2.1: Here, it is shown how the KS probability for the period distribution behaves when given simulated samples that are generated by using parameters that are exactly the same as, similar to, and completely different than an original simulation's parameters. See the text for details of the simulations.

\begin{tabular}{cccc}
\hline $\begin{array}{c}\text { TRIAL } \\
\#\end{array}$ & $\begin{array}{c}Q_{P} \\
(\text { SAME })\end{array}$ & $\begin{array}{c}Q_{P} \\
(\text { SIMILAR })\end{array}$ & $\begin{array}{c}Q_{P} \\
(\text { DIFFERENT })\end{array}$ \\
\hline 1 & 0.1299 & 0.0296 & $<10^{-12}$ \\
2 & 0.8928 & 0.0094 & $<10^{-12}$ \\
3 & 0.7397 & 0.1098 & $<10^{-12}$ \\
4 & 0.6958 & 0.1744 & $<10^{-12}$ \\
\hline AVG & 0.6145 & 0.0808 & $<10^{-12}$ \\
\hline
\end{tabular}

\subsection{Method for Comparison}

To compare these trial results quantitatively, the Kolmogorov-Smirnoff (KS) test, a non-parametric estimator based on the maximum deviation seen in the cumulative distribution of two sample data sets (see, e.g., Press et al., 1992) is used. For a given distribution, the KS test returns a statistic that is used to determine a probability, $Q$, that two samples came from the same underlying population. As described below, this probability is used to investigate whether model pulsar populations are inconsistent with the observed data.

To investigate the sensitivity of the KS test to changing model parameters, a single simulation using the optimal parameters from the FK06 model is run. Next, four further Monte Carlo realizations of the same model are generated and compared to the original simulation using the KS probability for the period distribution, $Q_{P}$. Then, a small modification is made, changing $\mu_{P}$ from $300 \mathrm{~ms}$ to $280 \mathrm{~ms}$, four more simulations are run, and $Q_{P}$ is computed by comparing with the original simulation. 
Finally, a more significant change to the model is made, changing $\mu_{P}$ to $250 \mathrm{~ms}, \sigma_{P}$ to $100 \mathrm{~ms}$, and $\mu_{\log B}$ to 12.00 . Four further simulations are run using these parameters and $Q_{P}$ is recorded against the original simulation. From the results of these simulations that are summarized in Table 2.1, it is concluded that individual KS probabilities above $10 \%$ suggest that two populations are consistent with another, while probabilities in the range 1-10\% imply possible differences between populations. However, the steep decline in the KS probability when two populations differ significantly suggest that it is a powerful metric to weigh one model against another.

In this work, it is desired to track multiple observed parameters relevant to these simulations. Therefore, following Bhattacharya et al. (1992), a figure of merit (FOM) is defined for each simulation:

$$
\mathrm{FOM}=\log \left[Q_{P} \times Q_{\dot{P}} \times Q_{l} \times Q_{b}\right]
$$

Here, the subscripts on each $\mathrm{KS} Q$ value refer to the distributions of period $(P)$, period derivative $(\dot{P})$, Galactic longitude $(l)$ and Galactic latitude $(b)$.

\subsection{Results and Discussion}

In this section, the results are described and discussed. The main goal is to compare the power law spin-down model (Equation 2.2) with the new spin-down model proposed by CS06 (Equation 2.9) under a variety of assumptions. The logical flow of the approach is summarized in Figure 2.2. 


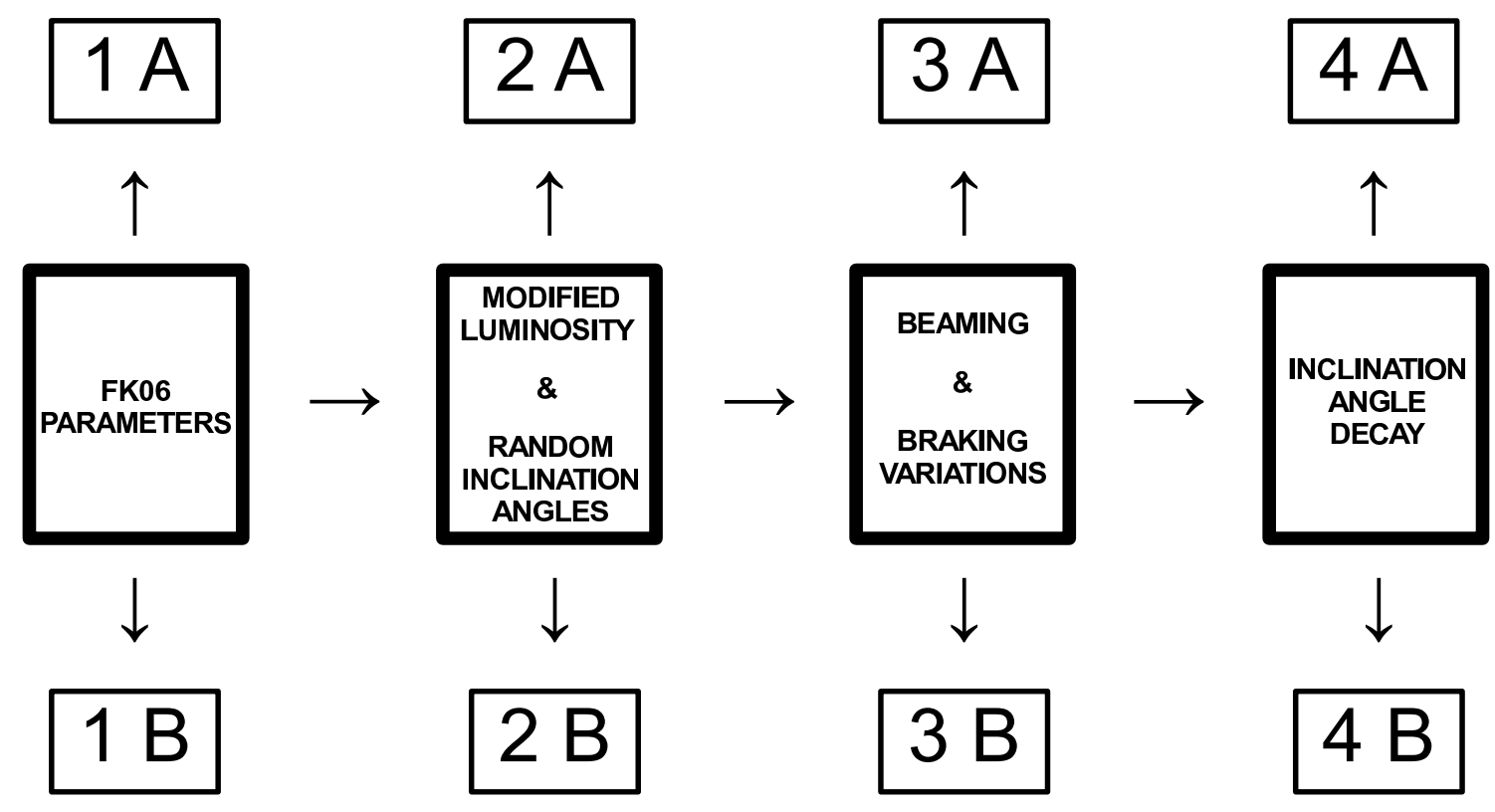

Figure 2.2: Schematic showing the logical flow of this approach to comparing the power law (A) and CS06 spin-down (B) models. Model 1 begins by using the best parameters from FK06. As described in the text, subsequent models then contain additional complexity in an attempt to be more physically plausible representations of the true population.

\subsubsection{Simulation Models}

The first simulation began by using the basic model parameters from FK06 and applied them to both spin-down laws which are called models $1 \mathrm{~A}$ and $1 \mathrm{~B}$. In this convention, "A" refers to the power-law spin-down model and "B" refers to the CS06 spin-down model. Model 2 attempts to improve the original model by changing the luminosity law and using random inclination angles to better match the observed sample. Subsequent models are motivated to be more physically realistic with the incorporation of: angle-dependent beaming models and a range of braking indices for models 3A and 3B, and inclination angle decay for models $4 \mathrm{~A}$ and $4 \mathrm{~B}$.

Table 2.2 provides an overall summary of the results, showing base-10 log- 
arithms of the individual KS probabilities and the FOM for each model. Also computed is the mean birth rate $(\mathcal{R})$ of pulsars required to produce the observed sample sizes for each model. This is defined as the ratio of the total number of pulsars generated in each simulation to the maximum age of the population, $t_{\max }$.

\subsubsection{Model 1: FK06 Basic Parameters}

Adopting the optimal parameters found by FK06, Model 1A was generated and is shown in Figure 2.3. The histograms show a relatively accurate replication of the original FK06 results. While the results of FK06 are generally able to be

reproduced, it is found that there are somewhat lower KS probabilities for $P$ and $\dot{P}$ for FK06. Obtaining an exact match to results obtained with a different simulation is challenging, and the discrepancies that are found highlight the difference in the implementation of the model in the two codes. Most of the major results are well duplicated. Some other parameters, such as $\alpha$ and $\beta$, had to be changed slightly from the values presented in FK06, but the majority of their results are obtained by using this code. These changes are addressed in the remaining models.

For Model 1B, all of the parameters of Model 1A are retained, with the exception of the spin-down law, which is replaced by an implementation of CS06 described in Section 2.2. As evidenced by the results shown in Table 2.2, the $P$ and $\dot{P}$ distributions are not as well reproduced.

This model had mixed results. Many areas, such as the effect of the alignment angle, the effect of having no angle dependence, and the underlying distribution of 

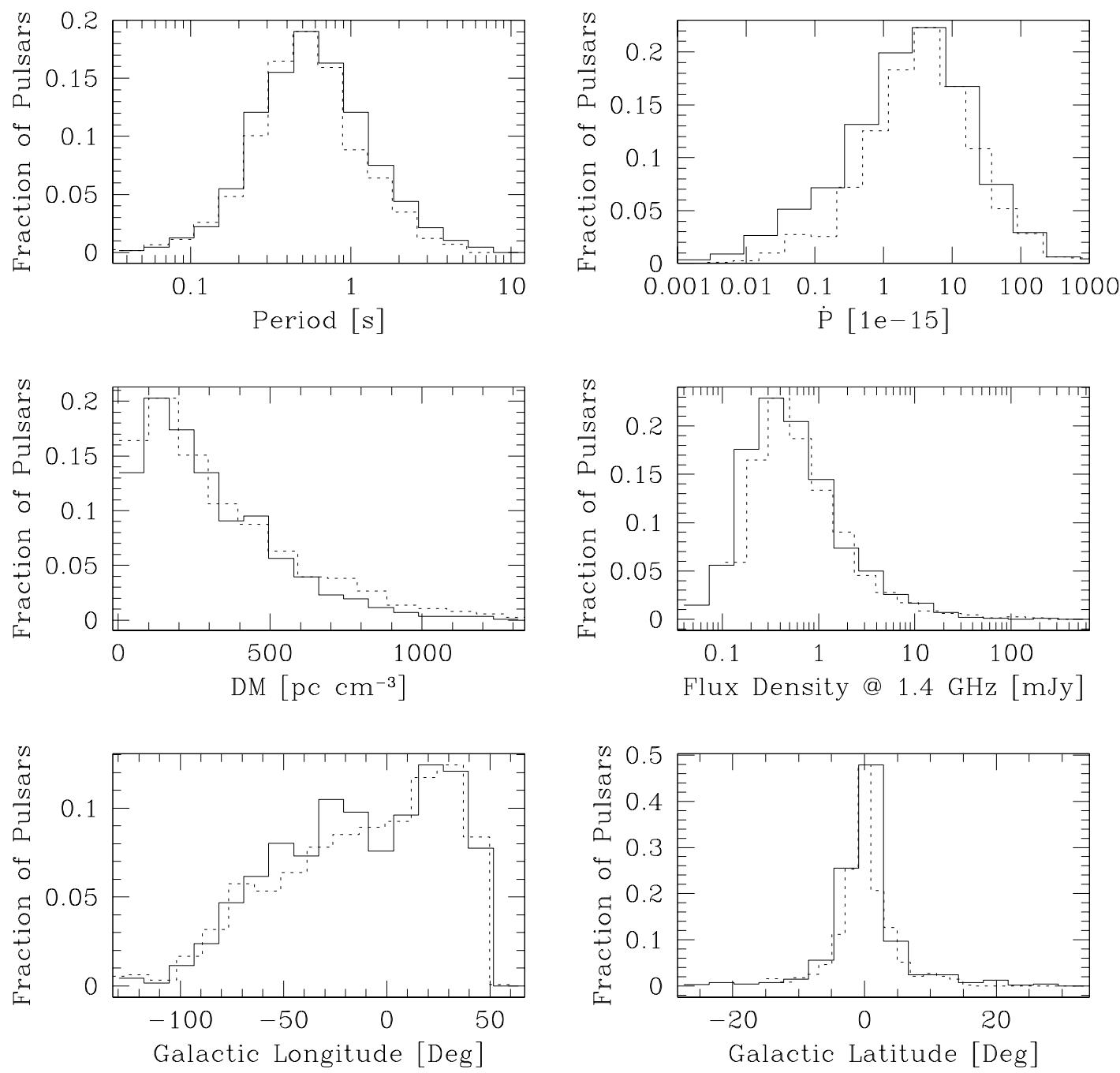

Figure 2.3: Histograms of some of the key properties from Model 1A. The solid histograms represent the observed pulsar population. The dotted histograms are the results of this simulation. 

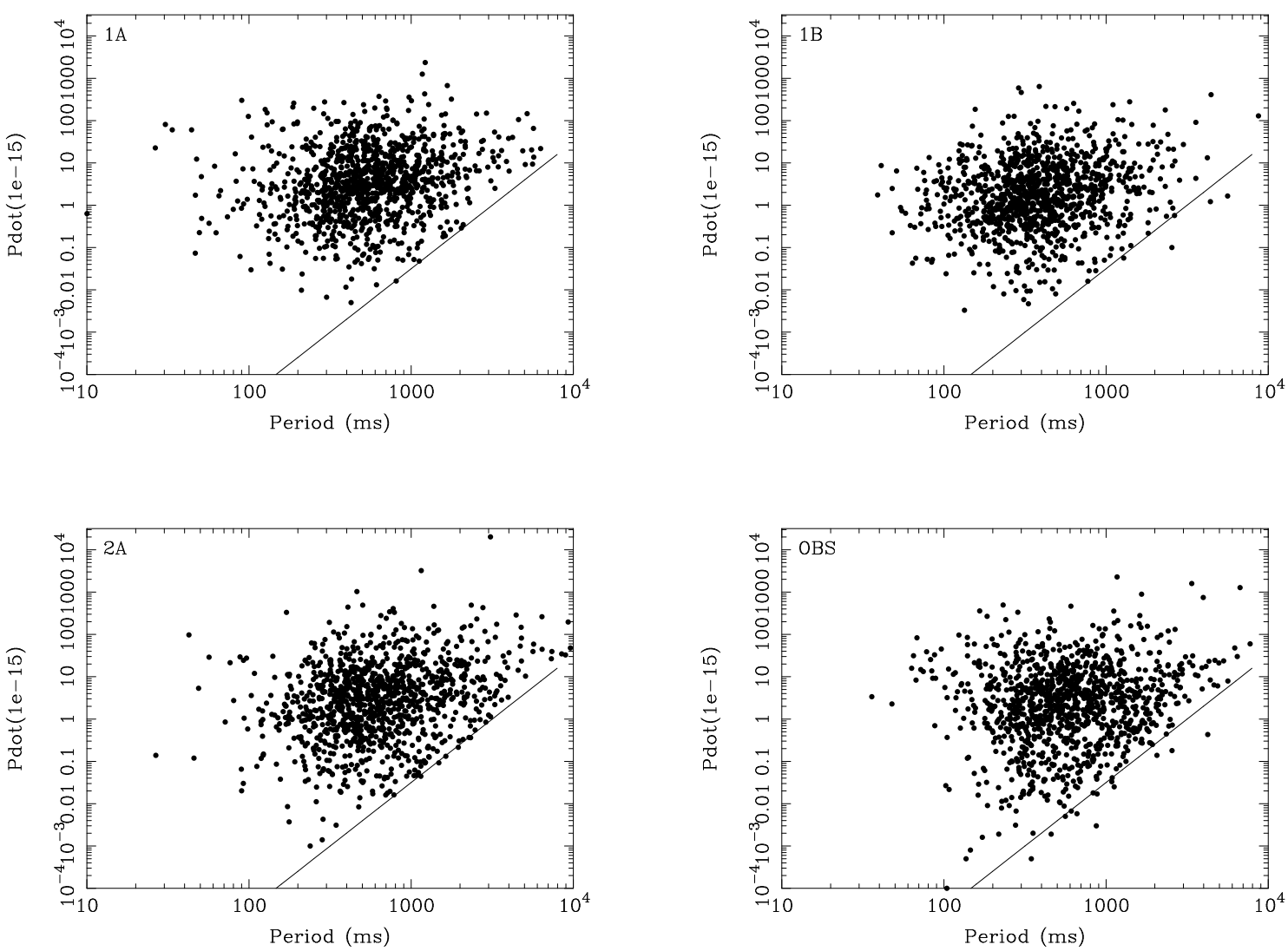

Figure 2.4: The resulting $P-\dot{P}$ diagrams, with the adopted death line shown in each plot for reference. Shown in the upper panels are the implementations of FK06 (model 1A, upper left) and CS06 (model 1B, upper right) using these simulations. The lower panels show the improved simulation (model 2A, lower left), which is based on an FK06 spin-down model, and the real observed sample of pulsars (lower right).

pulsars lying below the death line (see Figures 1, 3, 6 of their paper), were replicated with this code. However, the KS statistics were not as high as they are for the FK06 spin-down model. This applies not only to Model 1B, but to the rest of the B models as well. 
Table 2.2: Summary of KS statistics, figures of merit (FOM), and birth rate $(\mathcal{R}$, in psrs/century) comparing FK06 with the four models considered in this work. See text for details of the various models.

\begin{tabular}{lccccccccc}
\hline & \multicolumn{4}{c}{ Model 1 } & \multicolumn{2}{c}{ Model 2 } & \multicolumn{2}{c}{ Model 3 } & \multicolumn{2}{c}{ Model 4 } \\
Parameter & FK06 & \multicolumn{1}{c}{ A } & \multicolumn{1}{c}{ B } & \multicolumn{1}{c}{ A } & B & \multicolumn{1}{c}{ A } & B & \multicolumn{1}{c}{ A } & \multicolumn{1}{c}{ B } \\
\hline$P$ & -2.15 & -1.68 & -12.0 & -0.94 & -7.00 & -1.75 & -12.0 & -12.0 & -10.7 \\
$\dot{P}$ & -1.20 & -4.41 & -5.35 & -0.51 & -0.67 & -0.08 & -1.76 & -6.37 & -2.21 \\
$l$ & -0.90 & -0.12 & -0.12 & -0.07 & -0.09 & -0.28 & -0.40 & -0.10 & -0.25 \\
$b$ & -1.14 & -0.50 & -0.13 & -0.23 & -0.30 & -0.95 & -1.87 & -1.01 & -1.56 \\
\hline FOM & -5.39 & -6.71 & -17.6 & -1.75 & -8.06 & -3.06 & -16.0 & -19.5 & -14.7 \\
$\mathcal{R}$ & 2.8 & 3.85 & 1.97 & 3.56 & 2.84 & 2.97 & 3.75 & 5.31 & 3.68 \\
\hline
\end{tabular}

\subsubsection{Model 2: Modified Luminosity Law and Random Inclination}

\section{Angles}

In an attempt to improve the low KS statistics seen in the $P$ and $\dot{P}$ distributions for Model 1, a range of different luminosity indices were searched. Using Equation 2.14, $\alpha$ was varied from -1.9 to -0.8 and $\beta$ from 0.1 to 0.9 . The best result occurred when $\alpha$ and $\beta$ take the values of -1.0 and 0.5 , while FK06 found optimal values of -1.5 and 0.5 . As discussed in FK06, simulation techniques vary from model to model and small deviations may occur between models in the search for optimal parameters.

So far, orthogonal rotators have been assumed in the spin-down models. A more realistic and self-consistent approach is now followed by assigning random inclination angles to all pulsars.

The results from Model 2 were significantly better than Model 1, as seen in 
Table 2.2. At this point, it can be seen how much improvement is gained by changing only two parameters, so other parameters were tested to see if this improvement continued.

\subsubsection{Model 3: Beaming Model and Braking Index Variations}

Motivated by the improvements seen in Model 2, the requirement for the braking index $n=3$ was relaxed, and a range of braking indices was allowed. Based on the observed sample (see Section 1.2.4), a sensible choice was to select $n$ from a flat distribution in the range $1.5<n<3.0$.

The previous models adopt the TM98 beaming model, which is used in FK06. However, computing the beaming fraction based on a period-dependent opening angle law is now considered.

These results were worse than the previous model in terms of KS statistics, however, they were still better than the original model. For Model 3A, the birthrate decreased from nearly 4 pulsars per century to 3 per century, which is closer to the observed supernova rate of 2.5 per century (Tammann et al., 1994). However, Model 3B had just the opposite trend: the birthrate changed from 3 to 4 pulsars per century.

\subsubsection{Model 4: Inclination Angle Decay}

The final model was motivated by the recent results of WJ08, who found evidence for alignment of the spin and magnetic axes. In both Models 4A and 4B, 
the initially random inclination angles decay according to Equation 2.1 with an exponential decay timescale of $t_{d}=10^{7} \mathrm{yr}$. Model 4A used the spin-down law found in Equation 2.7 while Model 4B assumed the CS06 relation given in Equation 2.9. As shown in Table 2, both models performed very poorly, with significantly lower figures of merit than the earlier models without alignment. For the case of Model $4 \mathrm{~A}$, this poor agreement can be understood when it is seen that Equation 2.7 is essentially equivalent to an exponential decay of the magnetic field - more generally, an exponential decay of the braking torque. From the work of FK06, and preliminary simulations that have been carried out here, a decaying braking torque is inconsistent with the observations. For the case of Model 4B, the strong dependence on the spindown model (Equation 2.9) with $\chi$ is also the reason for the low figure of merit.

These results present a dilemma. On one hand, the empirical evidence for alignment presented by WJ08 appears to be very robust. On the other hand, it was found that magnetic dipole spin-down laws provide a good description of the population without inclination angle decay. The spin-down laws that have been implemented depend critically on this inclination angle. This implies that one way to resolve the discrepancy is to modify the spin-down law so that inclination angles are removed. While there is no physical basis for taking the $\sin ^{2} \chi$ term out of Equation 2.3, a modified version of Model 4A which uses Equation 2.5 instead of Equation 2.7 does produce improved results, though the overall FOM $(-6)$ is still lower than seen for the other models. In a future paper, this issue will be investigated further. Based on these current results, however, it appears that the braking torque on a pulsar is independent of its magnetic inclination angle. 
Finally, for completeness, Models 2A, 3A, and 4A were compared with the pulse width model mentioned in Smits et al. (2009). The birthrates for all three trials remained consistent with each model, however, the KS statistic FOM was generally worse. It was concluded that changing the way in which pulse widths are modeled would not improve these results.

\subsubsection{Individual Parameters}

Separate from the optimal models, various pulsar parameters were tested by themselves in order to gain a better understanding of how they affect the resulting pulsar population. In this section, each of these parameters are examined.

\subsubsection{Beaming}

Multiple simulations were run to test the beaming models. For each trial, only the beaming model which was to be tested was altered, and all other parameters were left the same. The constant beaming fraction model, or one in which $20 \%$ of all pulsars beam towards Earth, and the NV83 beaming model both gave poor results. The KS statistics using these models were very close to zero for all simulations.

The beaming models of LM88, B90, and TM98 each gave much better results. The TM98 model yielded the best population of pulsars, but it was not statistically better than the other two models. Finally, a period-dependent model was tried, similar to one described in WJ08. This gave the best overall result in terms of KS statistics and birthrates. 
Based on these results, a requirement for the population is a period-dependent beaming model, as well as small opening angles, similar to LM88, B90, and TM98. These three beaming models, along with WJ08, are all statistically about the same.

\subsubsection{Braking Index}

As a test of braking indices, simulation Model 2A was used. Indices ranging from 3.2 to 2.5 were tried, as well as a very realistic random model in which indices were chosen at random between 1.5 and 3.0. There were very few differences found when varying the braking indices, as shown in Table 2.3. Due to the lack of any significant difference between various braking indices, the realistic random model was adopted for use in these simulations.

It is useful to again look back at the A models from Table 2.2. There was a decrease in birthrate when going from Model 2 to Model 3. The two parameters that were altered between the two models were the braking index and the beaming model. The results presented in Table 2.3 make it clear that this drop in birthrate was not entirely due to the braking indices, and thus the angle-dependent beaming model must affect the birth rate as well.

\subsubsection{Luminosity}

Many luminosity simulations were run by changing the power law indices of $P$ and $\dot{P}$. The best result that was obtained yielded indices of -1.0 for $P$ and 0.5 for $\dot{P}$. The trials also included a simulation involving random luminosities, i.e. no 
Table 2.3: Results from 9 simulations with different values of the pulsars' braking indices are shown. This is an implementation of Model 2A, changing only the braking index during each simulation. The resulting birth rate, in units of pulsars born per century, and FOM (as described previously) is shown for comparison purposes.

\begin{tabular}{ccc}
\hline Braking Index & $\mathcal{R}$ (psrs/century) & FOM \\
\hline$n=3.2$ & 3.75 & -3.78 \\
$n=3.1$ & 3.99 & -4.09 \\
$n=3.0$ & 3.56 & -1.75 \\
$n=2.9$ & 3.51 & -1.63 \\
$n=2.8$ & 3.52 & -2.27 \\
$n=2.7$ & 3.53 & -3.66 \\
$n=2.6$ & 3.25 & -1.41 \\
$n=2.5$ & 3.28 & -2.74 \\
$1.5<n<3.0$ & 3.05 & -6.38 \\
\hline
\end{tabular}

period dependence. The results were less than ideal, and not close to the results that were obtained when using a period-dependent luminosity distribution. Thus, there must be some period dependency for the luminosity. Similar conclusions were reached by FK06, however, their optimal value for the $P$ power law index was -1.5 .

For the ideal simulations, the underlying luminosities have a distribution that is very similar to the log-normal distribution found in Figure 15 of FK06. In this regard, this distribution provides the best current estimate of the pulsar luminosity function. Unlike previous power-law models (see, e.g., Lorimer et al., 2006), the lognormal does not require a lower luminosity cutoff and is strongly recommended for studies which require some reasonable assumption about the luminosity function. 


\subsubsection{Covariances}

With such a large number of model parameters in these simulations, it is important to keep track of any interdependencies that might be expected. The biggest covariances that were found involve the luminosity. Changing the braking index along with the luminosity function can be done in such a way that two very similar results can be obtained with two completely different luminosity distributions and braking indices. For example, $n=3.0$ and power indices of -1.5 and 0.5 could yield the same results as $n=2.7$ and indices of -1.2 and 0.8 . A similar relationship was found between the luminosity and the alignment angle. Running a simulation with all angles equal to $90^{\circ}$ might give similar results to another simulation with all angles equal to $45^{\circ}$ and having luminosity indices of -1.8 and 0.9 . Most of these covariances are beyond the scope of this work, simply due to the large matrix of parameters that would be analyzed.

\subsubsection{Death Line}

For completeness, simulations of both models were run such that a death line was not used. As one might expect, there was an overabundance of high period pulsars that have very low luminosities. The results here confirmed that, within the framework of the models that have been developed here, a death line is indeed required when modeling a distribution of pulsars. While it might be possible to construct a model in the future which does not require a death line, there is not currently a straightforward means of achieving this. 


\subsection{Conclusions}

Two pulsar spin-down models have successfully been implemented and selection effects have been accounted for as far as possible to synthesize populations of observable radio pulsars. From a statistical comparisons of various models, there is now a better understanding of how their parameters affect the spin-down evolution of pulsars on a $P-\dot{P}$ diagram. The main conclusions are as follows:

- The magnetic dipole spin-down model from FK06 works best with these simulations. The FK06 results are able to be reproduced and a few results improved by modifying some of their parameters. The model of CS06 produces poorer results. Further modifications of this model appear to be required to improve it.

- The braking index, $n$, does not have a significant impact on these results. Having a braking index of $n=3.0$ works just as well as the rather unphysical scenario of $n>3.0$. For the optimal simulation, the most realistic model is used, which picks a random braking index between 1.5 and 3.0 for each pulsar.

- The optimal configuration for magnetic inclination angles is a random distribution. Models in which the inclination angle decays on timescales of $\sim 10^{7} \mathrm{yr}$ do not reproduce the observations well. Further modifications to the spindown laws appear to be required in order to account for the strong empirical evidence for alignment found by WJ08.

- Pulsar luminosities must have a period dependence. The use of a power law 
for the period and period derivative replicated the $P-\dot{P}$ diagram. By tweaking the exponents in that law, the resulting distribution of pulsars can be altered on this diagram. Even with period dependent luminosity laws and beaming models, a death line is required to explain the dearth of pulsars in the lower right of the $P-\dot{P}$ diagram.

Studying these relationships between the $P-\dot{P}$ diagram and the various pulsar parameters allowed covariances to be more apparent, eliminated a few parameter models, and overall obtained better insight to the behavior of pulsars as they evolve across the $P-\dot{P}$ diagram. Future studies will be able to further narrow down some parameter models and, in particular, allow for the investigation of the issue of magnetic alignment. 


\section{Chapter 3}

\section{Computer Simulations of the Magellanic Clouds Pulsar Population}

This chapter was originally published as a refereed paper in the Monthly Notices of the Royal Astronomical Society (MNRAS) by J. P. Ridley and D. R. Lorimer in June of 2010. Minor wording changes from the original accepted paper have been made where appropriate.

\subsection{Introduction}

The closest neighboring galaxies to the Milky Way, the Large and Small Magellanic Clouds (henceforth LMC and SMC), contain 19 known radio pulsars (Manchester et al., 2006). Since both the LMC and SMC represent a different star formation environment compared to the Milky Way and its globular cluster system, it is interesting to use these results to constrain the pulsar content in the clouds. So far, no millisecond pulsars (MSPs) have been found, though this is in part a result of the relatively low sampling rates and frequency resolutions of previous surveys (see Table 3.1). The aim of this work is to use Monte Carlo simulations to reproduce the results from previous LMC and SMC surveys, determine the most likely size of the normal pulsar population, place upper limits on the MSP population, and make predictions for future surveys of the clouds.

In Section 3.2, past surveys of the Magellanic Clouds are compared. Sec- 
Table 3.1: Observing parameters for the previous surveys considered in this paper, as well as the parameters for a currently ongoing survey using the BPSR (Berkeley-Parkes-Swinburne Data Recorder) pulsar backend at the Parkes Radio Telescope.

\begin{tabular}{lcccc}
\hline Parameter & $\begin{array}{c}\text { McConnell et al. } \\
(1991)\end{array}$ & $\begin{array}{c}\text { Crawford et al. } \\
(2001)\end{array}$ & $\begin{array}{c}\text { Manchester et al. } \\
(2006)\end{array}$ & $\begin{array}{c}\text { BPSR Survey } \\
(2009)\end{array}$ \\
\hline$t_{\text {obs }}(\mathrm{s})$ & 5000 & 8400 & 8400 & 8400 \\
$t_{\text {samp }}(\mathrm{ms})$ & 5.000 & 0.250 & 1.000 & 0.064 \\
$T_{\text {sys }}(\mathrm{K})$ & 60 & 21 & 21 & 20 \\
$f(\mathrm{MHz})$ & 610 & 1374 & 1374 & 1374 \\
$\Delta f(\mathrm{MHz})$ & 120 & 288 & 288 & 288 \\
$\Delta f_{\text {chan }}(\mathrm{MHz})$ & 2.5 & 3.0 & 3.0 & 0.1 \\
\hline
\end{tabular}

tion 3.3 contains a detailed description of these simulations and how they are able to reproduce results from the past surveys. From this model, the population of normal pulsars in the LMC and SMC can be inferred. New limits from these MSP simulations are presented in Section 3.4. Results are discussed and predictions are presented for future Magellanic Cloud surveys in Section 3.5. Finally, in Section 3.6, the main conclusions are drawn.

\subsection{Previous Surveys of the Magellanic Clouds}

Multiple radio surveys have been completed of both the Large and Small Magellanic clouds. The various features and results of each survey are discussed in this section. The survey data acquisition parameters are summarized in Table 3.1.

The original pulsar survey of the clouds was by McConnell et al. (1991) which detected three pulsars in the LMC and one in the SMC. After the success of the Parkes Multibeam Pulsar Survey (Manchester et al., 2001), searches of the clouds 
Table 3.2: List of all spin-powered radio pulsars in the LMC and SMC. It should be noted that the Crawford et al. survey did not observe the entire LMC, so their LMC results are not considered in these simulations. Also, PSR B0540-69 is the only currently known LMC pulsar that was not detected in any of these surveys.

\begin{tabular}{lccccc}
\hline PSR Name & Location & $\begin{array}{c}\mathrm{DM} \\
\left(\mathrm{pc} \mathrm{cm}^{-3}\right)\end{array}$ & $\begin{array}{c}\text { McConnell } \\
(1991)\end{array}$ & $\begin{array}{c}\text { Crawford } \\
(2001)\end{array}$ & $\begin{array}{c}\text { Manchester } \\
(2006)\end{array}$ \\
\hline J0045-7319 & SMC & 105.4 & $\mathrm{X}$ & $\mathrm{X}$ & $\mathrm{X}$ \\
J0455-6951 & LMC & 94.9 & $\mathrm{X}$ & & $\mathrm{X}$ \\
J0502-6617 & LMC & 68.9 & $\mathrm{X}$ & & $\mathrm{X}$ \\
J0529-6652 & LMC & 103.2 & $\mathrm{X}$ & & $\mathrm{X}$ \\
J0113-7220 & SMC & 125.5 & & $\mathrm{X}$ & $\mathrm{X}$ \\
J0535-6935 & LMC & 93.7 & & $\mathrm{X}$ & \\
B0540-69 & LMC & 146.5 & & & $\mathrm{X}$ \\
J0045-7042 & SMC & 70.0 & & & $\mathrm{X}$ \\
J0111-7131 & SMC & 76.0 & & & $\mathrm{X}$ \\
J0131-7310 & SMC & 205.2 & & & $\mathrm{X}$ \\
J0449-7031 & LMC & 65.8 & & & $\mathrm{X}$ \\
J0451-67 & LMC & 45.0 & & & $\mathrm{X}$ \\
J0456-7031 & LMC & 100.3 & & & $\mathrm{X}$ \\
J0519-6932 & LMC & 119.4 & & & $\mathrm{X}$ \\
J0522-6847 & LMC & 126.5 & & & $\mathrm{X}$ \\
J0532-6639 & LMC & 69.3 & & & 5 \\
J0534-6703 & LMC & 94.7 & & & 12 \\
J0543-6851 & LMC & 131.0 & & & \\
J0555-7056 & LMC & 73.4 & & & \\
\hline T0TALS & SMC & & & & \\
\hline
\end{tabular}

with greatly increased sensitivity were performed. Crawford et al. (2001) surveyed the SMC, finding two more associated pulsars. One is in the SMC while the second is in the LMC, found while searching for radio pulsations from an LMC X-ray pulsar. Manchester et al. (2006) extended this effort, surveying both the SMC and LMC. For the SMC, this survey discovered 3 new pulsars, while the LMC survey discovered 9 more normal pulsars. Currently, there are a total of 19 known spin-powered radio pulsars in the LMC and SMC. 


\subsection{Modeling the Normal Pulsar Population}

The goal is to create a working model of normal pulsars distributed throughout the Magellanic Clouds that can accurately reproduce the results found in each of the three surveys carried out so far. To do this, Monte Carlo simulations of the pulsar population are performed using an adapted version of the freely available PSRPOP software package ${ }^{1}$ (Lorimer et al., 2006).

The simulation begins by seeding each pulsar in the Magellanic Clouds. To do this for the SMC, a sphere with a radius of $2.0 \mathrm{kpc}$ centered at a Right Ascension of 0h $51 \mathrm{~m}$ and a Declination of $-73^{\circ} 07^{\prime}$ is considered. This sphere is then populated with pulsars using an exponential distribution (in the $\mathrm{x}, \mathrm{y}$, and $\mathrm{z}$ directions) having a scale length of $1.25 \mathrm{kpc}$ (Gonidakis et al., 2009). Similarly, the LMC simulations create a sphere with a radius of $4.5 \mathrm{kpc}$ centered at $05 \mathrm{~h} 17 \mathrm{~m}$ and $-69^{\circ} 02^{\prime}$ (Kim et al., 1998) and use an exponential distribution with a scale length of $1.50 \mathrm{kpc}$ (van der Marel, 2001).

The dispersion measure (DM) is randomly selected in the range $70-210 \mathrm{~cm}^{-3} \mathrm{pc}$. This is based on the DMs of other pulsars found in the Magellanic Clouds listed in Table 3.2. For the distance to each pulsar, values of $51 \mathrm{kpc}$ for the LMC (Koerwer, 2009) and $60 \mathrm{kpc}$ for the SMC (Harries et al., 2003) are used.

The period of each pulsar is then randomly generated from a log-normal distribution derived from a study of Galactic pulsars (Lorimer et al., 2006). For a period in $\mathrm{ms}$, the mean of the $\log$ of this distribution is 2.7 and the standard deviation is

\footnotetext{
${ }^{1}$ http://psrpop.sourceforge.net
} 
Table 3.3: Survey results for the three surveys considered in this work. The actual detections are compared to what the sample pulsar distributions predict to be detected. The first number is the actual number of pulsars detected, while the second number is the expected number of detections.

\begin{tabular}{lcc}
\hline SURVEY & SMC & LMC \\
\hline McConnell & $1 / 1$ & $2 / 3$ \\
Crawford & $2 / 2$ & NA \\
Manchester & $5 / 5$ & $12 / 12$ \\
\hline
\end{tabular}

0.34. Similarly, the radio luminosity is drawn from the log-normal function found by Faucher-Giguère \& Kaspi (2006); see also Chapter 2.

To determine the number of pulsars to generate, one set of survey parameters is used and an attempt is made to replicate their results. For these trials, the Manchester et al. (2006) survey is chosen because of its larger sample of detected pulsars. A sample distribution is created for both the LMC and the SMC. PSRPOP is run to generate enough SMC pulsars so that the Manchester survey would detect 5, and enough LMC pulsars so that it would detect 12. These two distributions are then used to analyze the McConnell and Crawford surveys.

Table 3.3 shows how the results of all three surveys are compared with what should be expected to detect. As can be seen, these results provide a self-consistent model of the Magellanic Cloud pulsars. Now, any future survey using this distribution of pulsars can be modeled and predictions can be made regarding what results should be obtained.

To determine the number of normal pulsars in the LMC and SMC, enough pulsars are generated to detect the corresponding number of pulsars found in each 


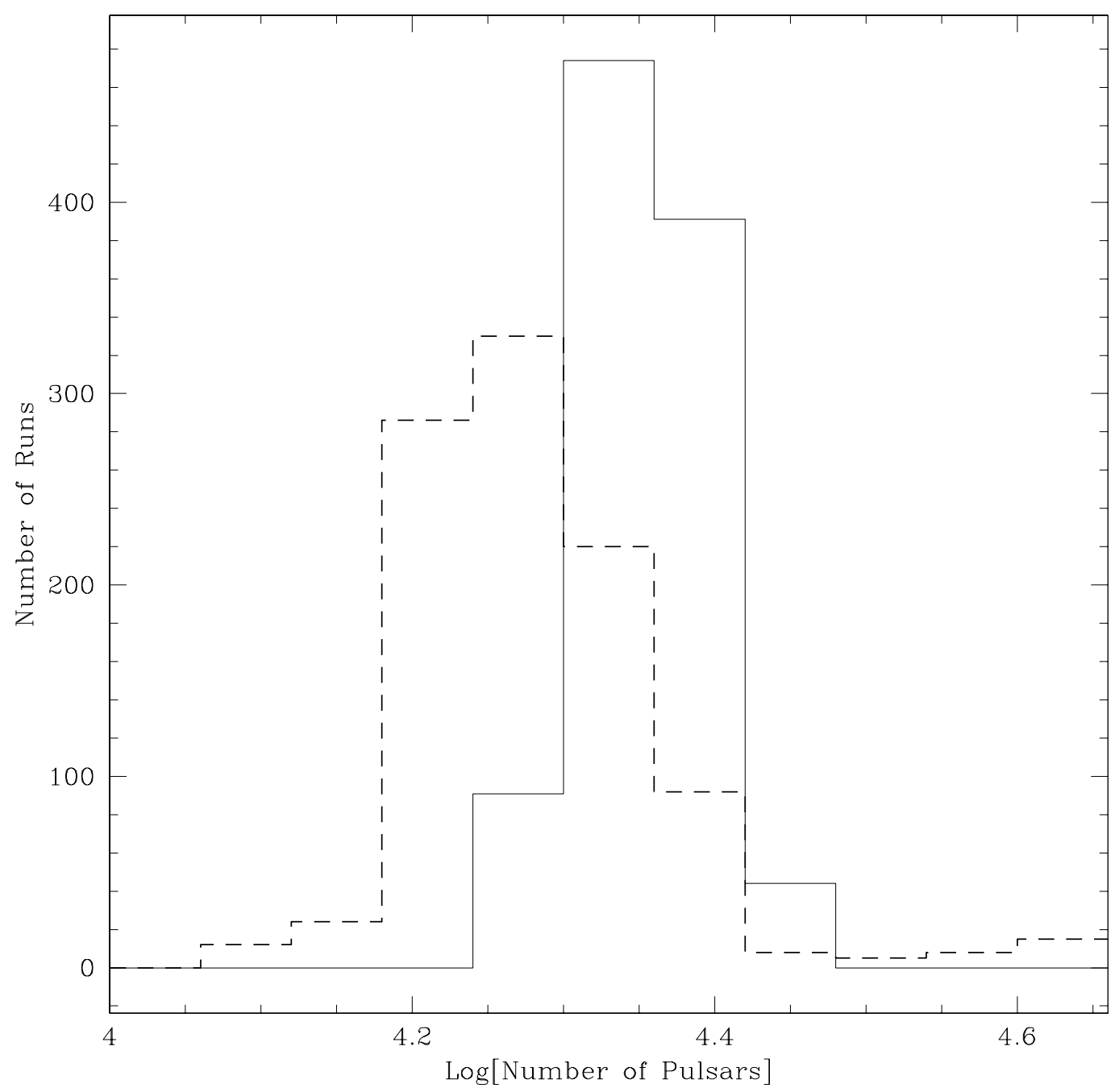

Figure 3.1: Distributions of the number of pulsars generated to model the LMC normal pulsars (solid), alongside the SMC pulsars (dashed).

cloud by the Manchester et al. survey. Repeating this process 1000 times creates a mean value for each cloud. The LMC has a mean value of $(1.79 \pm 0.20) \times 10^{4}$ pulsars, while the SMC has a mean of $(1.09 \pm 0.16) \times 10^{4}$. Figure 3.1 shows the distributions of normal pulsars produced by these simulations. 


\subsection{Modeling the Millisecond Pulsar Population}

Since no MSPs have been detected in the Magellanic Clouds, only an upper limit on the number of MSPs that could lie in the clouds can be found. As before, the Manchester et al. survey is used as a reference and a procedure similar to that described in the previous section is followed. All pulsar parameters are kept the same, with the exception of the period distribution. Here, a distribution of MSP periods used in a recent study (Smits et al., 2009) is used. For each of the LMC and SMC, PSRPOP is run again until the Manchester et al. survey detects one of the MSPs. The number of pulsars generated to get one detection will be the upper limit of the number of MSPs in the cloud.

To limit statistical noise and fluctuations, 1000 simulations are run, a histogram of the number of pulsars generated is created, and the mean is determined. To further limit these variances, the simulations are run to detect 10 MSPs and then the final number of pulsars generated is divided by 10. Figure 3.2 shows the simulation results.

To determine upper limits from these distributions, a point below which $95 \%$ of the simulations are contained is considered. For the LMC MSP population, an upper limit of 15,000 pulsars was found with $95 \%$ confidence. The equivalent simulations of the SMC yield somewhat higher numbers, presumably due to the fact that they are located at a farther distance from the Earth. The corresponding $95 \%$ confidence upper limit is 23,000 . 


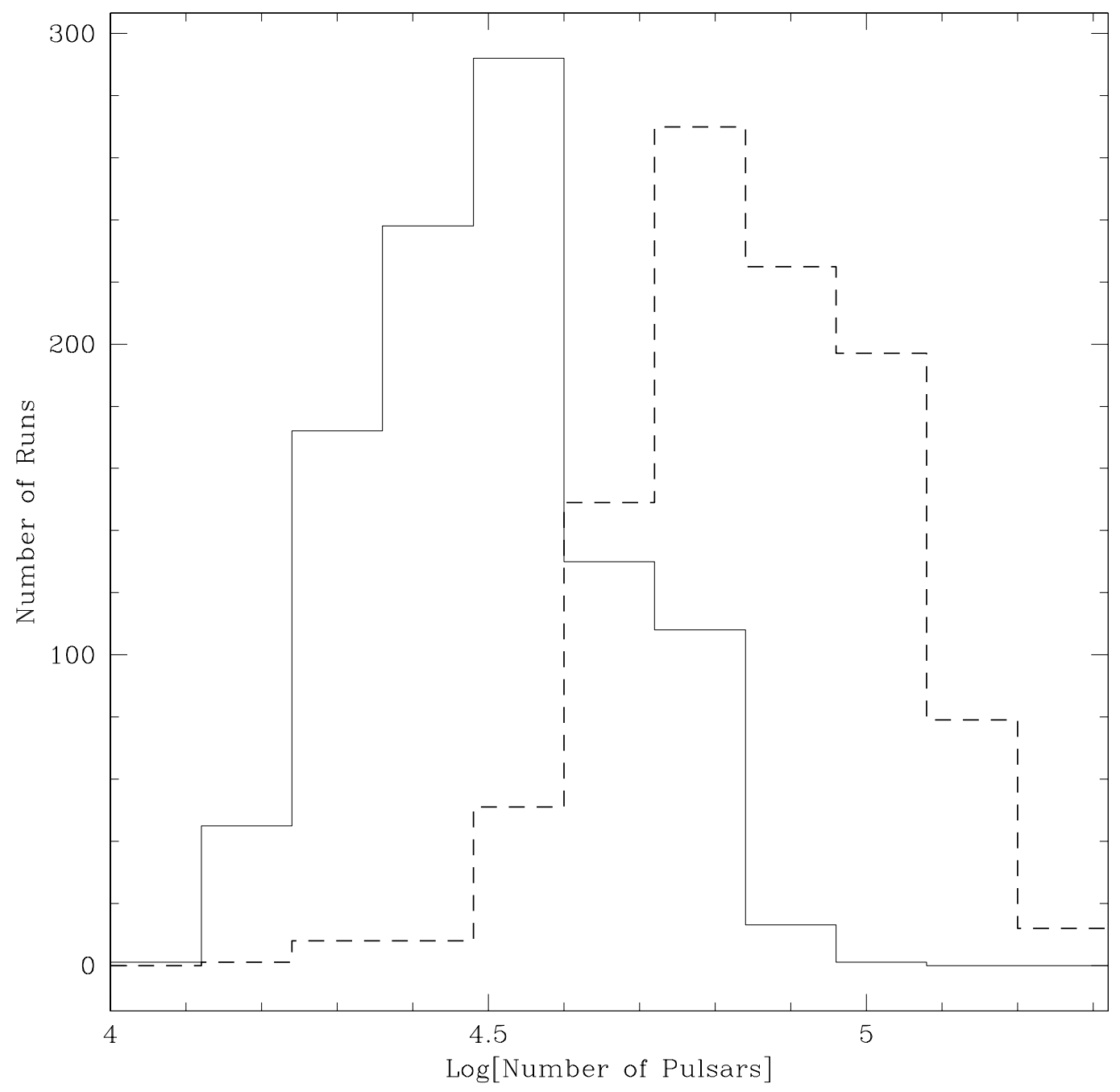

Figure 3.2: Similar to normal pulsar histograms, the distribution of pulsars generated for the MSP simulations of the LMC (solid) and SMC (dashed) are shown. 


\subsection{Discussion}

A self-consistent model of the normal pulsar population in the LMC and SMC has been produced. Now, some straightforward inferences from these results are discussed and the yields of future Magellanic Cloud surveys are predicted.

\subsubsection{Birthrates of Normal Pulsars}

To estimate the birthrate of normal pulsars in the Magellanic Clouds, the results from Faucher-Giguère \& Kaspi (2006) are used to obtain an average lifetime for Galactic pulsars. When their estimated number of pulsars of $(1.2 \pm 0.3) \times 10^{6}$ is divided by their Galactic pulsar birthrate of $2.8 \pm 0.1$ pulsars per century, an average radio lifetime of $(4.3 \pm 0.1) \times 10^{7}$ years is obtained. To calculate the birthrates in the LMC and SMC, the respective number of pulsars is divided by this average lifetime, and then multiplied by a factor to correct for those pulsars whose beams are not visible due to beaming effects. This factor is approximately 10 when averaged over the normal pulsar population (Tauris \& Manchester, 1998). These calculations lead to birthrates of $0.42 \pm 0.03$ and $0.25 \pm 0.03$ pulsars per century for the LMC and SMC respectively.

These birth rates, however, are likely to be underestimates of the true value due to the fact that both the LMC and SMC have relatively small escape velocities. Using values for the mass and radius of the SMC from Bekki \& Stanimirović (2009), an escape velocity $v_{\mathrm{esc}}=\sqrt{2 G M / R}$, of roughly $120 \mathrm{~km} \mathrm{~s}^{-1}$ is calculated. Similarly, an escape velocity for the LMC of $157 \mathrm{~km} \mathrm{~s}^{-1}$ is found. Given the high birth 
velocities known for Galactic pulsars (e.g. Hobbs et al., 2005; Faucher-Giguère \& Kaspi, 2006), potentially a large number of pulsars that are born in the clouds will eventually lie outside of the surveyed area within the age in which they are expected to be radio loud.

To quantify the impact of this effect on the birthrates, a simple simulation was run in which the velocity of each pulsar was chosen from a Maxwellian distribution with a 1-D dispersion of $265 \mathrm{~km} \mathrm{~s}^{-1}$ (Hobbs et al., 2005). After randomly selecting the age of the pulsar to be between 0 and $4.3 \times 10^{7}$ years, the distance the pulsar has traveled was calculated by multiplying the age and the velocity. Assuming that the pulsar starts at the center of either cloud, the final position is computed to determine if the pulsar has remained within the surveyed area of the cloud (radii of $5.6^{\circ}$ and $2.2^{\circ}$ for the LMC and SMC) for the duration of its lifetime. It was found that nearly half of all pulsars born in the LMC (45.9\%) escape the cloud, while $67.2 \%$ of all pulsars born in the SMC escape. The population of ejected radio pulsars that are active from both Magellanic Clouds is therefore substantial, of order $3.5 \times 10^{5}$ objects

The above calculations lead to effective birthrates of 0.8 pulsars per century for both the LMC and SMC. Given the uncertainties involved in making these corrections, the best conclusion that can be reached from the data at hand is that radio pulsar birth rates are comparable and likely to be somewhere in the range of 0.5-1 pulsar per century in both the LMC and SMC.

Tammann, Löffler \& Schröder (1994) carried out an extensive study of the Galactic and extragalactic supernova rate. They find a combined rate of type Ib and 
II supernovae of 0.45 and 0.11 per century in the LMC and SMC respectively. While the rate for the $\mathrm{LMC}$ is consistent with the estimate above, the pulsar birthrate in the SMC appears to be significantly higher than these supernova rates. In spite of this discrepancy, a simple exercise using Poissonian statistics shows that both pulsar and supernovae populations are consistent with the observation of one supernova in the LMC and zero supernovae in the SMC during the past 300 years of observations.

\subsubsection{Predictions for Future Surveys}

Previous surveys were limited in their ability to detect millisecond and rapidly rotating pulsars by the low data sampling rates and frequency resolution. A current survey of the Magellanic Clouds is making use of a new data acquisition system, BPSR, developed for the Parkes High Time Resolution Universe Legacy Survey ${ }^{2}$ (Keith et al., 2010) which is currently undertaking a large-scale search for pulsars along the Galactic plane. The parameters of this new, high-resolution pulsar data acquisition system for this new search of the Magellanic Clouds are summarized in Table 3.1. As can be seen, the time resolution is improved by factors of 4 and 16 over the Crawford et al. and Manchester et al. surveys, while the frequency resolution is improved by a factor of 30 .

With the MSP upper limits as references, sample distributions of the LMC and SMC MSP populations are created, and an attempt is made to detect the pulsars using the new survey parameters. Using $8400 \mathrm{~s}$ integrations, these simulations predict that the new survey will find at most 3 MSPs in each of the two Magellanic Clouds.

\footnotetext{
${ }^{2}$ http://astronomy.swin.edu.au/pulsar/?topic=hlsurvey
} 
With a full survey of the LMC and SMC, the absence of MSP detections would lower the upper limits on the number of MSPs to 5,500 and 8,000, respectively.

A pilot survey of the SMC began in May 2009 using this new system. The goal of this new survey is to detect or place stringent limits on the population of MSPs and giant-pulse emitting neutron stars in the Magellanic Clouds. Following the survey pointings from Crawford et al. (2001), giant pulses from PSR B0540-69 have been observed, and three previously known pulsars have been redetected. Further details of this survey are given in Chapter 5 .

Future predictions can be made that make use of the Square Kilometre Array (SKA). Following the "A" implementation (Smits et al., 2009) of the SKA, survey parameters are chosen that allow the LMC and SMC to be searched for both normal and millisecond pulsars. With a sky coverage per pointing of nearly $250 \mathrm{deg}^{2}$, the LMC can be covered in only 4 pointings, while the SMC can be completely searched with only 3. Using 10 minute integration times would keep the total survey time for either region under 1 hour and results in 2,500 and 1,400 normal pulsar detections in the LMC and SMC respectively. Additionally, this SKA survey could potentially detect 850 and 800 MSPs in the LMC and SMC, which is a considerable improvement over previous and current surveys of the Magellanic Clouds.

\subsection{Conclusions}

The results from three past Magellanic Cloud surveys were successfully reproduced, creating an accurate normal pulsar population for both the LMC and SMC. 
These populations provide estimates of the number of normal pulsars located in the $\mathrm{LMC}$ and $\mathrm{SMC}$ of $(1.79 \pm 0.20) \times 10^{4}$ and $(1.09 \pm 0.16) \times 10^{4}$ pulsars respectively. Taking into account pulsars whose beams do not intersect the Earth's line of sight, and the significant fraction of pulsars which escape both the LMC and SMC, it was found that the mean radio pulsar birth rate is most likely to be in the range $0.5-1$ pulsars per century in either cloud. This is consistent with historical observations of supernovae in the Magellanic Clouds. It is also found that a substantial population of radio pulsars will be ejected from the clouds and populate the local intergalactic medium.

Extending the analysis to the MSP population yields upper limits of 15,000 for the LMC and 23,000 for the SMC. If the current SMC survey at Parkes produces no MSP detections, that upper limit will be reduced to 8,000. Consequently, it can realistically be expected that no more than 3 MSPs will be detected in that survey. Also, if the current SMC survey extends to the LMC, a maximum of 3 MSPs would be detected, but a lack of MSP detections would reduce the MSP upper limit to 5,500 .

The results here, along with the future results of the ongoing BPSR survey (see Chapter 5) will continue to constrain the number of pulsars, both normal and millisecond, found in the Magellanic Clouds. With improved simulation techniques and more sensitive surveys, the true pulsar population will be able to be modeled with increased accuracy to allow for further research in this area. A logical extension of this work would be a full dynamical model of the pulsar content in the Magellanic Clouds as has been recently carried out for radio pulsars in the Milky Way Galaxy 
(Faucher-Giguère \& Kaspi, 2006; Ridley \& Lorimer, 2010). 


\section{Chapter 4}

\section{Searching for Pulsars}

\subsection{Introduction}

From choosing the correct telescope to accounting for various physical phenomena, there is a considerable amount of time and work that is required to search

for pulsars. In this chapter, the most common pulsar searching techniques and methods are explained. Figure 4.1 shows the main steps when searching for pulsars. Particular attention will be given to the computational analysis performed on the data and to the differences between each of the search techniques.

\subsection{Obtaining and Formatting the Data}

After observing a certain area of the sky, the data obtained by the telescope are stored in filterbank files. These files contain a collection of frequency channels versus time and are commonly referred to as "fil" files. Within the file, each frequency channel shows the intensity of the signal measured at that particular frequency as it varies over the length of the observation. Before these channels can be searched for evidence of pulsars, the filterbank file must now be formatted to correct for the presence of the interstellar medium (ISM) and, if desired, the motion of the Earth around the Sun. 


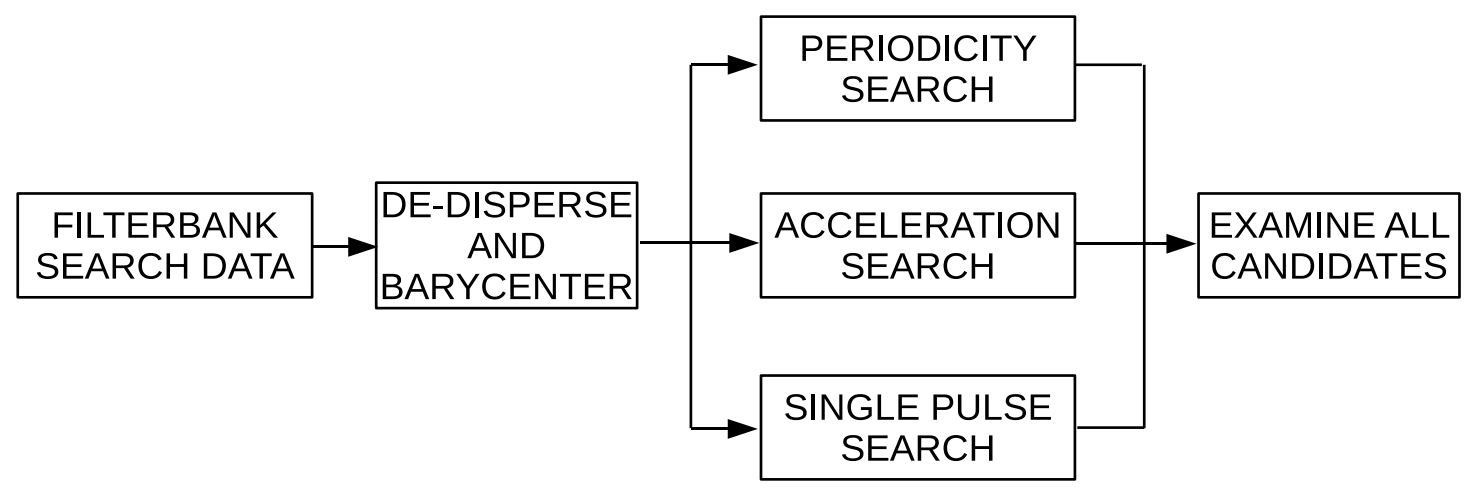

Figure 4.1: The main steps in pulsar searching. Each step is discussed in more detail later in this section.

\subsubsection{Barycentering}

The Earth's motion around the Sun can distort the signal that is received from the pulsar. To correct for this, the data files are barycentered, or adjusted so that the time stamps in the file represent the time that the signal would have been received at the Sun instead of at the telescope. This is especially important for comparing observations taken at different times of year, when the location of the Earth with respect to the Sun has changed significantly.

The barycentering process begins with a filterbank file and creates two running clocks. The first clock ticks at equal intervals according to the sampling time of the 
Table 4.1: This is a fake time series for a 1.2 second observation. Having six time samples in the topocentric data file would mean that the sampling time is 0.2 seconds. If the topocentric and barycentric clocks differed by greater than the sampling time after every third sample, an additional time sample (with a value of 0) would be added. After barycentering the data, the nine time samples would create a sampling time of 0.13 seconds in the new filterbank file. This is a gross exaggeration and is for demonstration purposes only, as most 30 min observations would only have a few samples added or removed.

\begin{tabular}{cc|cc}
\hline $\begin{array}{c}\text { Time Sample } \\
\text { (Topocentric) }\end{array}$ & $\begin{array}{c}\text { Intensity Level } \\
\text { (arbitrary units) }\end{array}$ & $\begin{array}{c}\text { Time Sample } \\
\text { (Barycentric) }\end{array}$ & $\begin{array}{c}\text { Intensity Level } \\
\text { (arbitrary units) }\end{array}$ \\
\hline 1 & 5842 & 1 & 5842 \\
2 & 5495 & 2 & 5495 \\
3 & 5486 & 3 & 0000 \\
4 & 5684 & 4 & 5486 \\
5 & 5247 & 5 & 5684 \\
6 & 5364 & 6 & 0000 \\
& & 7 & 5247 \\
& & 8 & 5364 \\
& & 9 & 0000 \\
\hline
\end{tabular}

observation and is known as the topocentric clock. The second, or barycentric clock, ticks with intervals equal to the sampling time multiplied by a factor which is calculated based on the position of the Earth and the Sun. If the topocentric clock runs slower than the barycentric clock, then an additional time sample is added to the resulting filterbank file. Similarly, if the topocentric clock runs faster than the barycentric clock, a time sample is removed from the data. The end result of barycentering a filterbank file is to create a new filterbank file with a slightly different sampling time. Table 4.1 shows how the time samples are changed during the barycentering process. 


\subsubsection{De-dispersion}

It was discussed earlier that Galactic signals get dispersed as they pass through the ISM, resulting in higher frequencies arriving sooner than lower frequencies. To compensate for this smearing, the data files must be de-dispersed. This is done by shifting each frequency channel in time so that all signals in all channels will arrive at the same time. The amount of the shift depends on the difference in frequency between each channel and the DM that is being searched. Since the distance to (and therefore the DM of) the pulsar is usually unknown, the standard practice is to search through a range of DMs starting at 0 and continuing to a predetermined maximum value which usually depends on the location of the sky that is being searched.

The effect of choosing an incorrect DM value is that the Signal-to-Noise ratio $(\mathrm{S} / \mathrm{N})$ of the pulsar's signal will be significantly reduced. Additionally, pulsars with very short periods (i.e. MSPs) are affected even more due to the DM smearing being such a large fraction of the pulse width. The effective pulse width can be calculated as

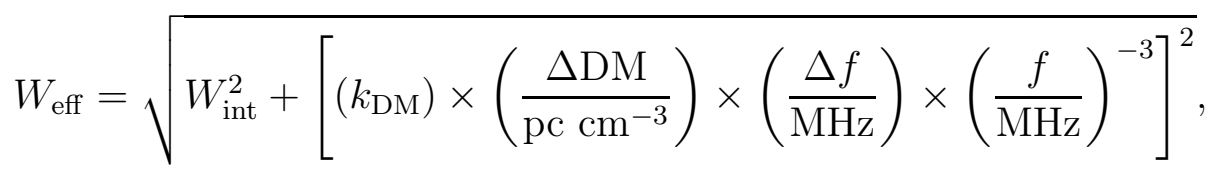

where $k_{\mathrm{DM}}=8.3 \times 10^{6} \mathrm{~ms}, W_{\text {int }}$ is the intrinsic pulse width, $\Delta f$ is the channel bandwidth, and $f$ is the center observing frequency. The subsequent influence on 
the $\mathrm{S} / \mathrm{N}$ due to the effective pulse width is:

$$
\mathrm{S} / \mathrm{N} \propto \sqrt{\frac{\mathrm{P}-\mathrm{W}_{\text {eff }}}{\mathrm{W}_{\text {eff }}}} .
$$

In order to prevent a pulsar's signal from being lost due to DM smearing, a DM step size must be accurately chosen to both avoid redundant computer processing and be sensitive to low period pulsars. Using Equations 4.1 and 4.2, Figure 4.2 shows how the choice of DM affects the $\mathrm{S} / \mathrm{N}$ of pulsars with varying periods.

The time delay, $\Delta t$, that occurs due to 2 differing frequencies is given by Equation 1.9 where $f_{1}$ and $f_{2}$ are the lower and upper frequencies. The minimum amount of time delay that the observations are sensitive to is the sampling time, $\mathrm{t}_{\mathrm{samp}}$, so if the bandwidth is defined as $\Delta f=f_{2}-f_{1}$ and the center frequency is defined as $f=\left(f_{1}+f_{2}\right) / 2$, the minimum DM step size can be calculated to be

$$
\left(\frac{\Delta \mathrm{DM}}{\mathrm{pc} \mathrm{cm}}-3\right)=1.205 \times 10^{-7}\left(\frac{t_{\mathrm{samp}}}{1 \mathrm{~ms}}\right)\left(\frac{f}{1 \mathrm{MHz}}\right)^{3}\left(\frac{\Delta f}{1 \mathrm{MHz}}\right)^{-1} .
$$

This will assure that the DM value to be searched will be close enough to the true value so that the pulsar's signal will not be missed. The original fil file has now been transformed into one time series for each DM (each time series is known as a "tim" file), which contain the de-dispersed data that will be searched.

Once the data files have undergone these processes, they are now ready to be searched using one or more of the following pulsar search methods. 


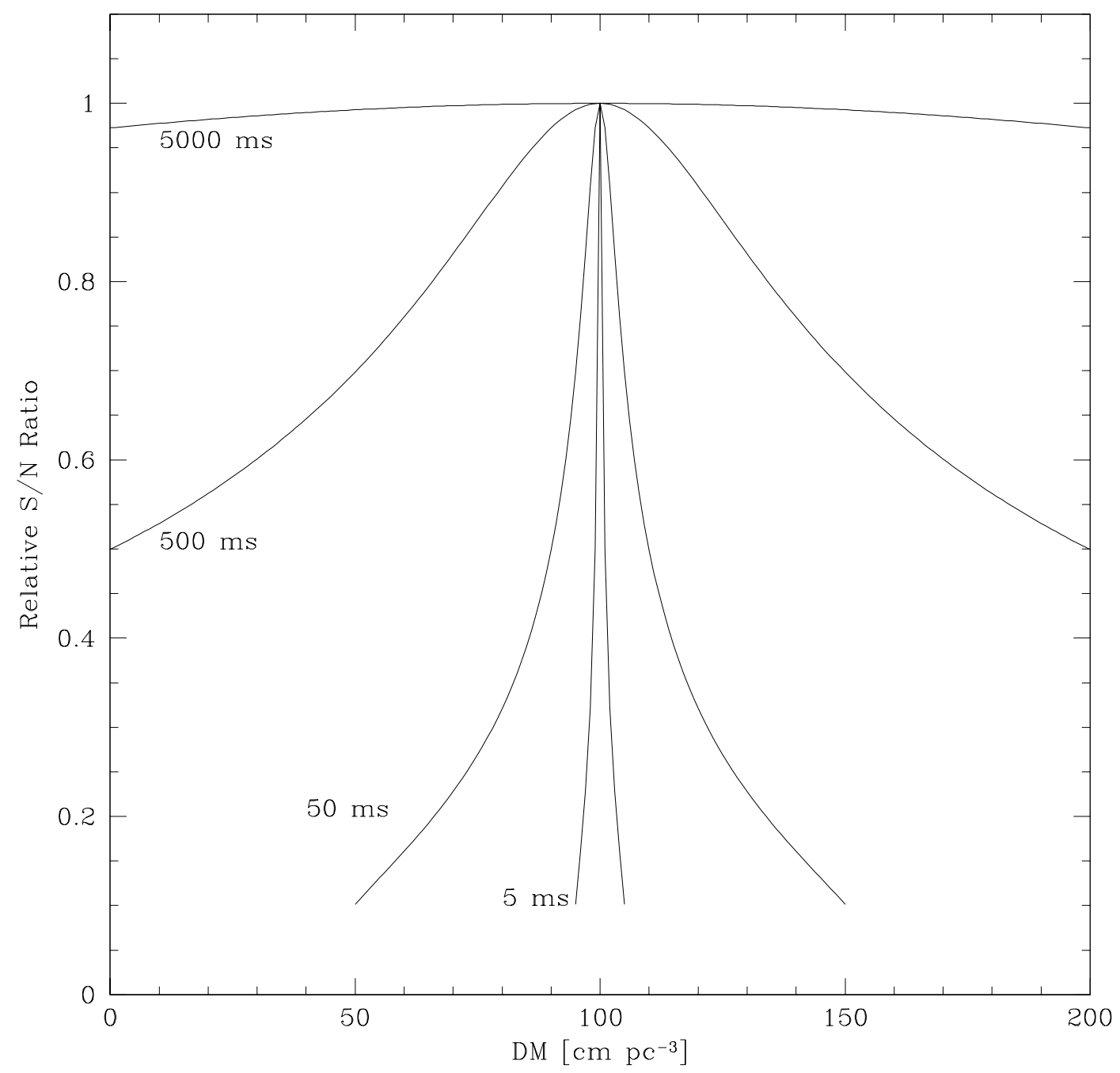

Figure 4.2: The relative $\mathrm{S} / \mathrm{N}$ ratio as a function of $\mathrm{DM}$ for 4 different pulse periods. Assuming a $8 \mathrm{MHz}$ bandwidth centered at $430 \mathrm{MHz}$, a pulsar with an intrinsic pulse width of $5 \%$ and a true DM of $100 \mathrm{pc} \mathrm{cm}^{-3}$ will have a reduced S/N when an incorrect DM value is chosen. This effect is most notable for pulsars with very short periods. 


\subsection{Periodicity Searches}

The trademark signature of a pulsar is the periodicity of its signal. Pulsars which rotate at the same rate (for the duration of a single observation) emit their sig-

nals at equally spaced intervals. The fact that the pulses arrive at these predictable times make pulsars some of the most accurate clocks in the Universe. Periodicity searches take advantage of these periodic signals to discover pulsars hidden by noise in the data.

To perform a periodicity search, the properly formatted data file is run through a program that sifts out all of the periodic signals. This is done by using a Fourier transform (Bracewell, 1999). Typically, Fourier Transforms (FTs) transform a continuous data set from one domain into another. For example, the continuous Fourier Transform can transform data from the time domain to the frequency domain using

$$
g(\omega)=\int_{-\infty}^{\infty} f(t) \exp (-i \omega t) d t
$$

where $f(t)$ is the original time series and $g(\omega)$ is the transformed frequency domain function. When performing a periodicity search, the FT will transform the time domain into the frequency domain. A power spectrum can be created with the transformed data in which each frequency will have a corresponding power level. The frequencies with the highest signal strength will appear to have the highest power level in the power spectrum. After a Fourier transform is performed, determining the strongest signals is as easy as finding the tallest spikes on the power spectrum 
graph.

Since the time series, $\tau_{n}$, generated by de-dispersing a filterbank file is composed of individual data points, the Discrete Fourier Transform (DFT) can be used. The $x^{\text {th }}$ component of the DFT can be represented as

$$
F_{x}=\sum_{n=0}^{N-1} \tau_{n} \exp (-2 \pi i x n / N)
$$

where $i=\sqrt{-1}$ and $N$ is the number of time samples. Thus, the frequency of the $x^{\text {th }}$ Fourier component, $\nu_{x}=x / T$, where $T=N t_{\text {samp. }}$. The width of each frequency bin is $1 / T$ and the highest frequency component is the Nyquist Frequency, $\nu_{\mathrm{Nyquist}}=1 /\left(2 t_{\mathrm{samp}}\right)($ Lorimer \& Kramer, 2005).

A major limitation of the DFT is that it requires $N^{2}$ computations to complete the transformation. To help reduce the amount of computing time, a fast Fourier Transform (FFT) can be used. The FFT is an algorithm that computes the DFT with a reduced number of computations, most notably being done with $N \log N$ computations (Press et al., 1992).

Another drawback to the DFT is the scalloping effect. Since the DFT transforms a time series into frequencies bins with center frequencies of $\nu_{x}=x / T$ and width $\Delta \nu=1 / T$, it is not as sensitive to frequencies that differ from those center frequencies. There are several methods available to help regain the sensitivity lost from the non-central frequencies. For more information on these methods and how the DFT has been modified for optimal use in pulsar searches, see Ransom et al. (2002). 
Most signals (especially those with very small pulse widths) do not have all of their Fourier power levels associated with a single, fundamental frequency. Much of this power is divided across several harmonics. As a result, the power seen at the fundamental frequency will be appearing at a somewhat lower level. One way in which to recoup some of this power is to undergo a procedure called harmonic summing (Taylor \& Huguenin, 1969). With harmonic summing, the amplitudes of higher harmonics are added to the power spectrum of the fundamental frequency. This is done by stretching the spectrum by a factor of 2 and then adding this stretched spectrum to the original spectrum. While this increases the noise by a factor of $\sqrt{2}$, the addition of the harmonics (each $\mathrm{n}^{\text {th }}$ harmonic gets a new spectrum stretched by a factor of $n$ ) directly add to the peaks of the fundamental frequency and significantly increase the S/N (Lorimer \& Kramer, 2005).

After the time series has been completely transformed, all signals found (now known as candidates) must be sorted according to their S/N. A typical minimum threshold for $\mathrm{S} / \mathrm{N}$ is between 7 and 10 . This threshold is determined by calculating the false-alarm probability that the $\mathrm{S} / \mathrm{N}$ will exceed a minimum value. In the presence of Gaussian noise, this minimum value can be approximated by:

$$
\mathrm{S} / \mathrm{N}_{\min } \approx \frac{\sqrt{\ln \left(\mathrm{n}_{\text {trials }}\right)}-0.88}{0.47},
$$

where $n_{\text {trials }}$ is the number of independent $\mathrm{S} / \mathrm{N}$ estimates in the search (Lorimer \& Kramer, 2005). All Fourier components that are analyzed from each trial DM are counted to obtain the number of $\mathrm{S} / \mathrm{N}$ estimates. Any candidates below this 
minimum value are deemed to be too insignificant to be completely distinguishable from background noise, and thus only the higher $\mathrm{S} / \mathrm{N}$ candidates will be considered.

After a candidate passes the $\mathrm{S} / \mathrm{N}$ test, it must be filtered further, usually by period and DM. Many low DM $\left(<5 \mathrm{pc} \mathrm{cm}^{-3}\right)$ candidates can be written off as radio interference, or Earth-generated signals. Also, periods of less than $1 \mathrm{~ms}$ are generally ignored, as this is below the theoretical minimum period limit. When the number of candidates reaches a number that can be realistically processed in a timely manner (which varies, depending on the available computer processing power and human work hours), they are then ready for the final processing stage.

Each candidate is now folded using the resulting DM and period. Folding effectively breaks the "tim" file into time segments that are equal to the length of the pulsar's period. These segments are then added on top of each other in an effort to amplify the signal and minimize the background noise. In general, a longer observation will produce a more amplified signal. The end result of the folding process is a plot that contains the pulse profile, $\mathrm{S} / \mathrm{N}$ graphs, and various other parameters, as described in Figure 4.3. Once this plot has been created, it is up to the pulsar searcher to examine all areas of the plot by eye and determine whether or not the candidate is indeed a pulsar. Some of the checks include making sure that the signal is visible in all frequency channels and throughout the entire observation, has believable periods, period-derivatives, and DMs, and has a pulse profile that is similar to other known pulsars. If all of these tests have passed, then follow-up observations of the candidate should be considered. Examples of candidates that would not pass these tests are shown in Figures 4.4 and 4.5. 

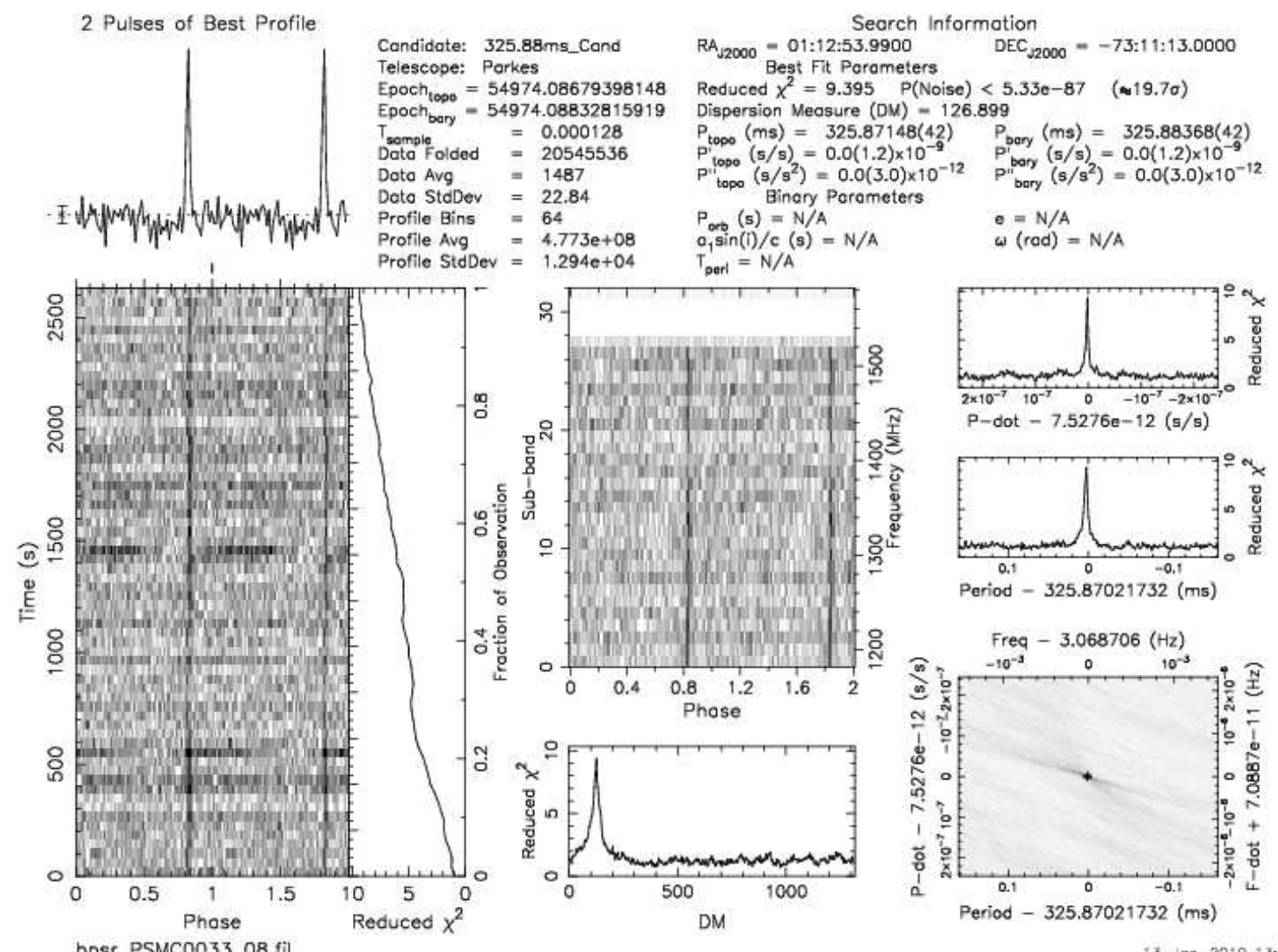

13- $\operatorname{sen}-2010 \cdot 13: 07$

Figure 4.3: A sample periodicity search result detecting the pulsar J0113 - 7220 . The top left plot shows two pulses of the best profile of the candidate so that the entire pulse phase can be observed. Parameters of the candidate and the search itself are found at the top right. The plot in the left column shows the intensity of the signal has a function of pulse phase and time, as well as how the reduced $\chi^{2}$ varies with time. The central plot shows the signal intensity as a function of pulse phase and frequency. The top sub-bands are missing because the receiver could only sample the bottom $340 \mathrm{MHz}$ (see Chapter 5). The bottom middle plot shows the reduced $\chi^{2}$ varies with DM. The three plots in the right hand column show reduced $\chi^{2}$ versus $\dot{P}$ and $P$, as well as $\dot{P}$ versus $P$. This plot was created using the PRESTO ${ }^{2}$ processing suite. 


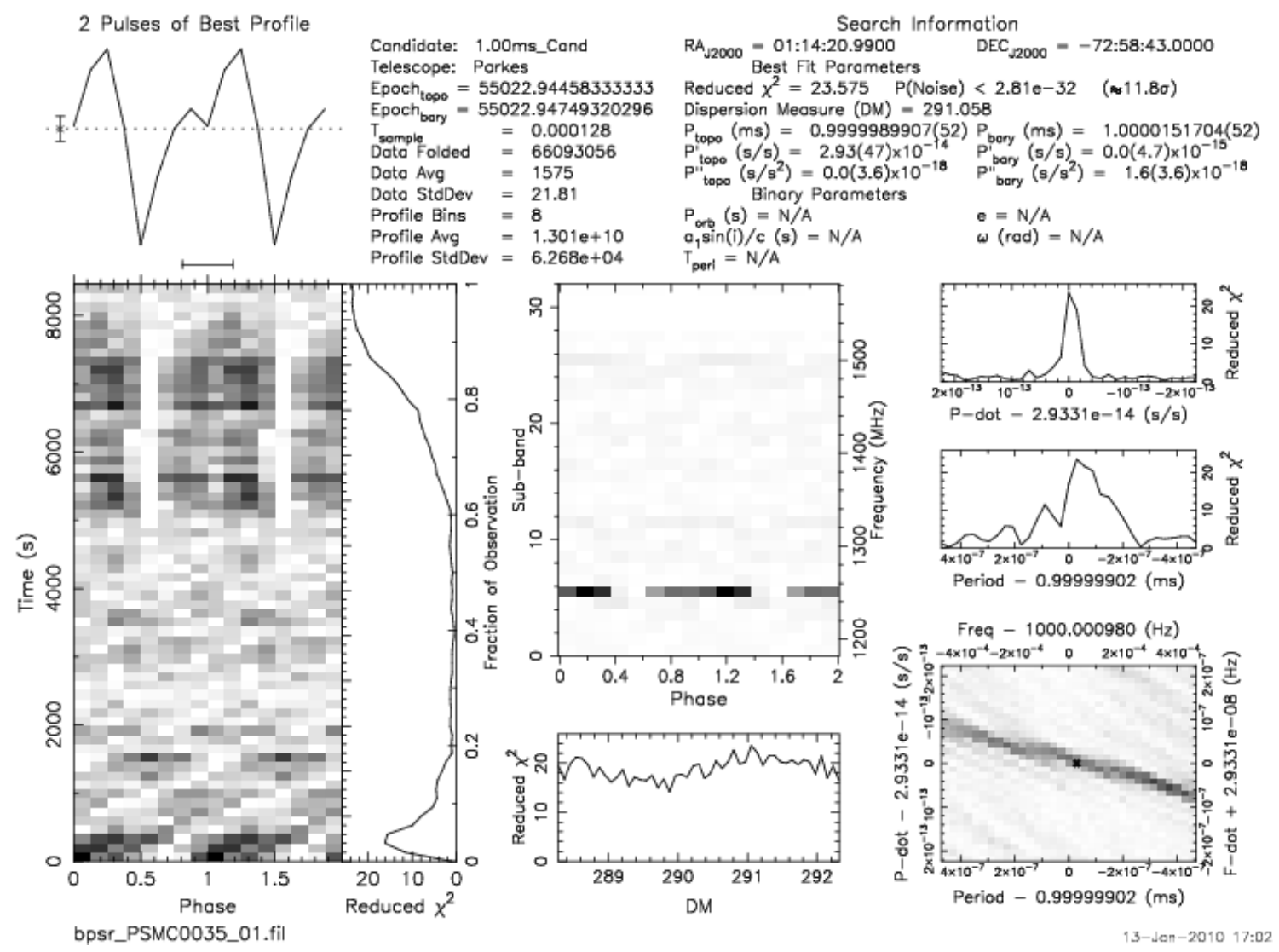

Figure 4.4: This candidate does not have a visible signal throughout the entire observation, nor is the signal visible across the entire frequency band. Also, there is not a clearly defined peak in the DM plot.

\subsection{Single Pulse Searches}

In addition to detecting pulsars based on the periodicity of their signals, it is also possible to detect them if a few of their pulses are significantly brighter than the underlying noise. These individual pulses are usually visible over a small range of DM values. This so-called single pulse searching provides another tool for detecting previously unknown pulsars. 


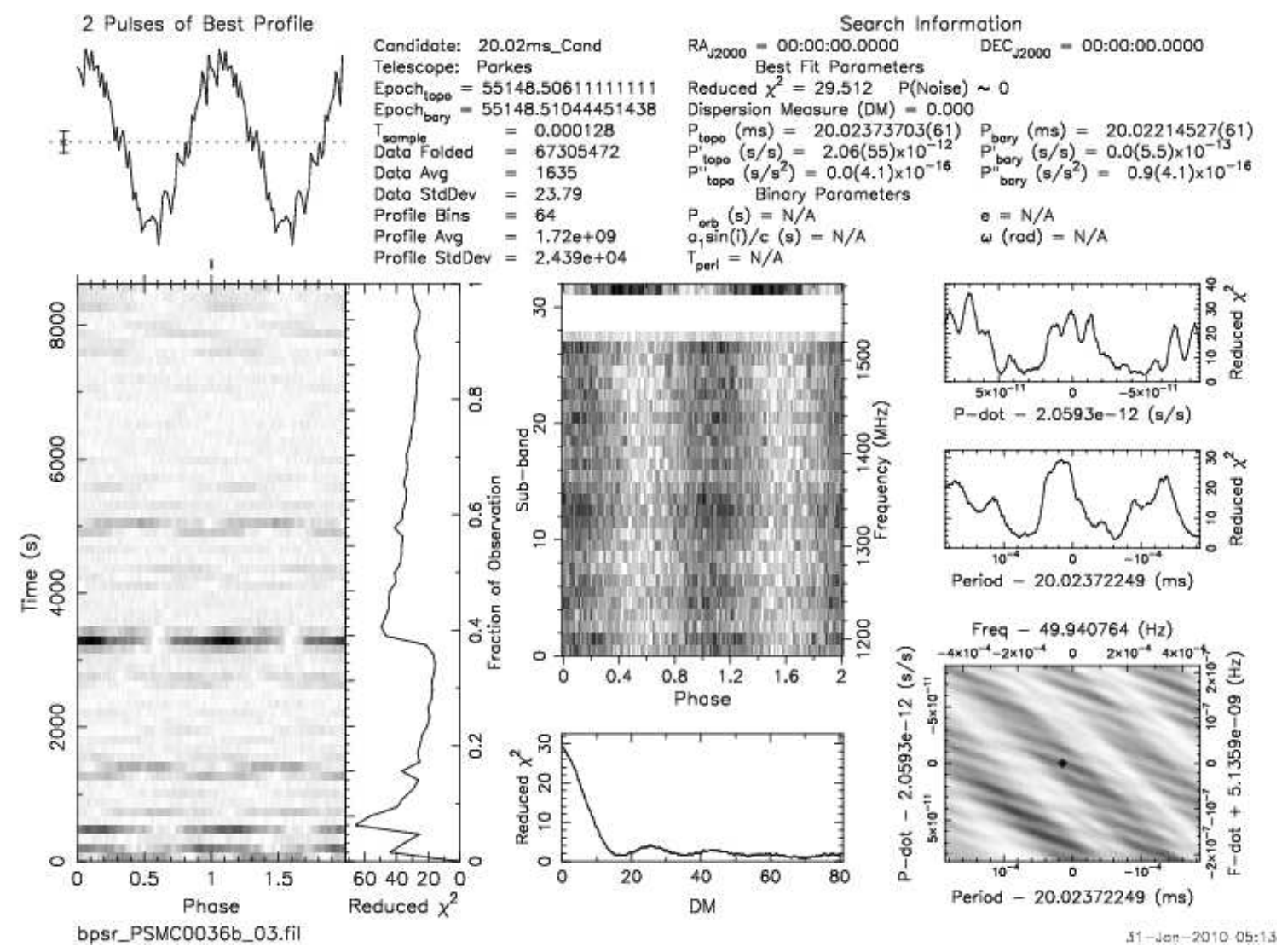

Figure 4.5: This candidate shows the result of the telescope detecting RFI from a local source. Note that the signal is only visible for a small range of times, the optimal DM is 0 , and the frequency of the signal is very close to the frequency used by the Australian power grid $(50 \mathrm{~Hz})$.

\subsubsection{Matched Filtering}

To detect individual pulses, one must look for signals that lie a few standard deviations above the mean signal. Each previously generated time series is individually searched for these pulses and this is done by a process called matched filtering. By convolving the time series with a predetermined pulse template (usually a Gaussian) and successively adding adjacent time samples, matched filtering is able to detect pulses of varying widths (Cordes \& McLaughlin, 2003).

A sample single pulse plot for B0540-69 is shown in Figure 4.6. In this plot, a 


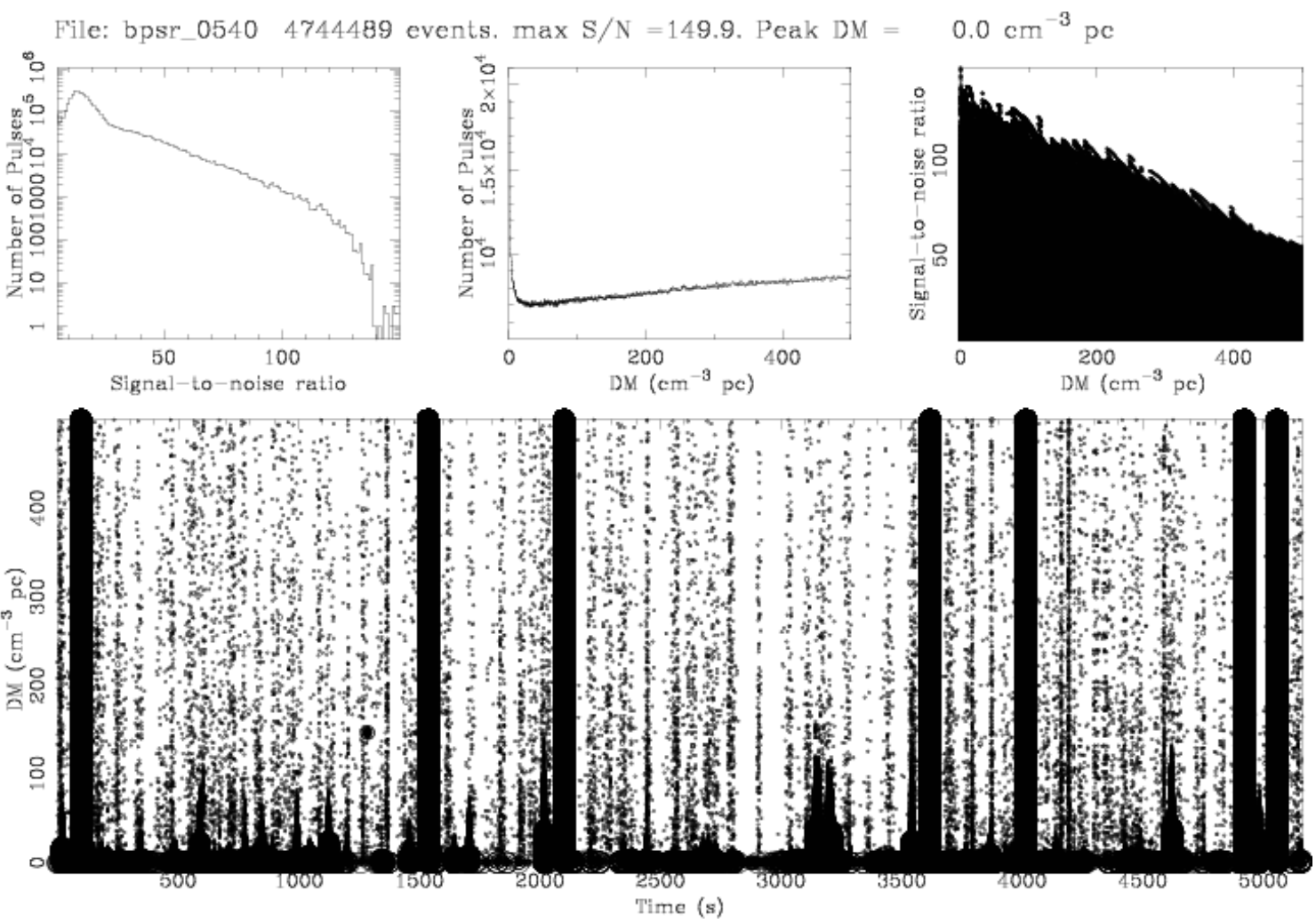

Figure 4.6: A single pulse plot showing the detection of a giant pulse emitted by B0540-69, seen at a DM of $146.5 \mathrm{pc} \mathrm{cm}^{-3}$ and a time of $1400 \mathrm{~s}$. The top plots show the number of events at a given $\mathrm{S} / \mathrm{N}$ ratio and a given $\mathrm{DM}$, as well as the $\mathrm{S} / \mathrm{N}$ ratio of the events at their respective DMs. The bottom plot shows the DM of each event as they appear in the time series. The size of each symbol is proportional to the strength of the observed pulse.

single pulse is observed, among a significant amount of radio frequency interference (RFI), near the $1400 \mathrm{~s}$ mark at a DM of $146.5 \mathrm{pc} \mathrm{cm}^{-3}$. This pulsar is known to occasionally emit giant pulses such as these, which are much stronger than its normal pulses (Johnston \& Romani, 2003). Many periodicity searches would not be able to detect this pulsar, as the normal pulse intensities are too weak to be detected. A single pulse search, therefore, is sometimes the only way to detect a pulsar (see, e.g. Deneva et al. (2009)). 


\subsubsection{Radio Frequency Interference}

As evidenced by the overwhelming display of detections in the single pulse plot of B0540-69, RFI causes significant problems for pulsar searchers. Much of the RFI is present at a DM of $0 \mathrm{pc} \mathrm{cm}^{-3}$ and is continually visible at all other DMs at that given time. To alleviate this problem, RFI can be removed from the observations by various methods. The simplest method is to remove all signals that appear to originate at a DM of $0 \mathrm{pc} \mathrm{cm}^{-3}$ and carry through to all other DMs. The obvious drawback of this is the loss of signal intensity, however, the gain is very apparent when looking at a single pulse plot that has undergone an RFI removal procedure (see Figure 4.7).

Another method of removing RFI from data is known as mean subtraction. With mean subtraction, the average intensity from all frequencies for each time sample is calculated. Then, this mean value is subtracted from each individual frequency channel for that time sample. Since most RFI occurs at a DM of $0 \mathrm{pc} \mathrm{cm}^{-3}$ and is not dispersed, the added intensity from the RFI will be mostly removed when the mean intensity is subtracted from the data (Eatough et al., 2009).

\subsection{Acceleration Searches}

Many pulsars are found in binary systems. With orbital periods on the order of days or even hours, the pulsars undergo accelerations that significantly affect how their signals are observed. Depending on whether the pulsar is moving away from or toward the observer, the emitted signal will be Doppler shifted, making the 

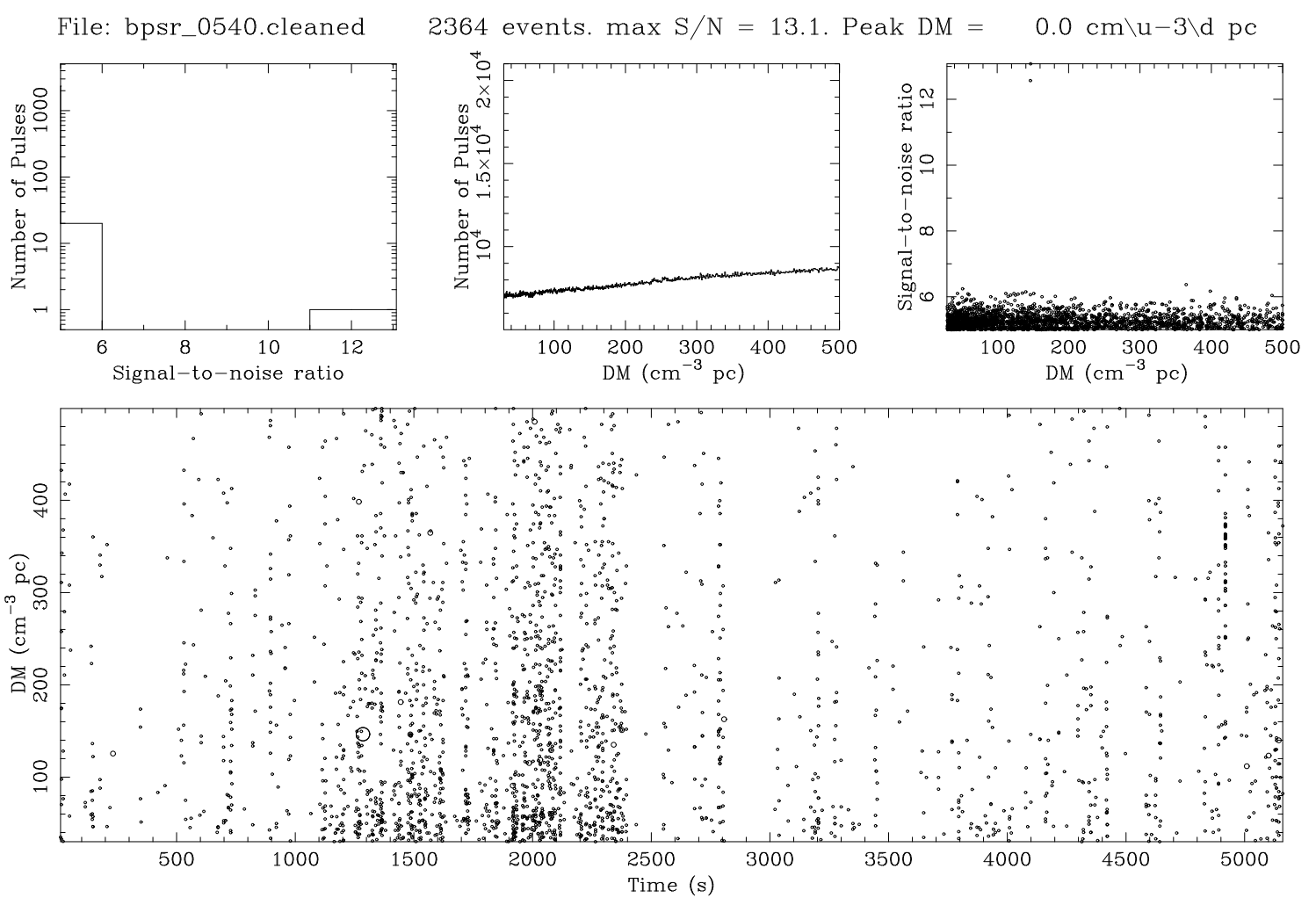

Figure 4.7: A single pulse plot with the RFI removed from the same observation of B0540-69. The detection looks much weaker, but is much more visible without the overpowering nature of RFI. The pulse is now clearly visible in the $\mathrm{S} / \mathrm{N}$ plots with a value of 12 .

observed period greater or smaller than the true period. An acceleration search is performed to compensate for this phenomenon, which attempts to detect the pulsar by transforming the observed signal in a reference frame which is ideally at rest with respect to the pulsar.

To first order, the observed time interval, $\tau(t)$, is related to the time interval in the reference frame of the pulsar, $\tau_{0}$, via the usual Doppler formula

$$
\tau(t)=\tau_{0}\left(1+\frac{V(t)}{c}\right)
$$


where $V(t)$ is the radial velocity of the pulsar along the line of sight of the observer. In principle, it is possible to calculate $V(t)$ from the Keplerian orbital parameters (see Lorimer \& Kramer (2005)), but since those parameters are often unknown, a 5dimensional parameter space must be searched to accurately constrain the velocity. Unfortunately, this method is very computationally intense, so an easier solution is required. By assuming a constant orbital acceleration, $a$, the velocity can be written as $V(t)=a t$. This simple approximation allows for a 1-dimensional search to be performed.

Visiting the Doppler effect again, the true spin frequency of the pulsar, $\nu_{0}$, and the observed spin frequency, $\nu(t)$, are related by

$$
\nu(t)=\nu_{0}\left(1-\frac{V(t)}{c}\right) .
$$

Taking the time derivative of the observed spin frequency and recalling that $V(t)=$ $a t$, it is found that

$$
|\dot{\nu}|=\frac{a \nu_{0}}{c} .
$$

This change is frequency will cause the signal to drift across multiple Fourier bins when analyzed by taking a Fourier transform. To calculate the number of bins across which the signal drifts, consider an observation of length $T$, which then has Fourier bins of size $\Delta \nu=1 / T$. The number of bins, $N_{\text {drift }}$, that the signal will drift across is just equal to the change in frequency of the pulsar due to the orbital motion divided 
by the width of one Fourier bin times the length of the observation, or

$$
N_{\mathrm{drift}}=\frac{\dot{\nu}}{\Delta \nu} T=\frac{a \nu_{0} T^{2}}{c}
$$

Ideally, the signal should drift by less than 1 bin, so $\Delta N_{\text {drift }}<1$ requires that $\Delta a<$ $c /\left(T^{2} \nu_{0}\right)$, or $\Delta a=c P / T^{2}$, where $P$ is the spin period of the pulsar. With a fixed observation length and a minimum period to be searched, this optimal acceleration step size makes it possible to run normal periodicity searches across a wide range of accelerations to detect pulsars that would previously be undetectable due to such large changes in their spin periods.

Most binary pulsars have accelerations around to $5 \mathrm{~m} \mathrm{~s}^{-2}$. However, some pulsars, such as B2127+11C $\left(9.5 \mathrm{~m} \mathrm{~s}^{-2}\right)$ and B1744-24A $\left(33 \mathrm{~m} \mathrm{~s}^{-2}\right)$, have been found to have accelerations that are significantly greater (Camilo et al., 2000).

There are, however, a couple of limitations to this approach. First, consider the line-of-sight velocity to a pulsar in a binary orbit,

$$
v(t)=A \omega \sin i \sin (\omega t+\phi)
$$

where $\omega=2 \pi / P_{b}, P_{b}$ is the orbital period, $i$ is the inclination angle of the orbit, $\phi$ is the starting phase of the orbit, and $A$ is a constant derived from Kepler's Laws:

$$
A=\frac{M_{2}}{M_{1}+M_{2}}\left[\frac{G\left(M_{1}+M_{2}\right) P_{b}^{2}}{4 \pi^{2}}\right]^{\frac{1}{3}} .
$$

Here, $M_{1}$ and $M_{2}$ are the masses of the pulsar and its companion, respectively, and 
$G$ is the gravitational constant. Equation 4.11 can be differentiated with respect to time to give the acceleration that a pulsar in a binary orbit would undergo:

$$
a(t)=A \omega^{2} \sin i \cos (\omega t+\phi) .
$$

As evidenced by this equation, a face-on orbit (i.e. $i=0^{\circ}$ ) yields no acceleration, while an edge-on orbit $\left(i=90^{\circ}\right)$ produces the maximum acceleration. It should also be noted that very small orbital periods can produce very large accelerations, which significantly affect the detectability of pulsars. Johnston \& Kulkarni (1991) simulated the range of detectable pulsars based on their orbital and spin periods and quantified their results in a quantity known as $\gamma$. This value represents the degradation of the $\mathrm{S} / \mathrm{N}$ of an accelerated pulsar over a pulsar moving with constant velocity. This means that

$$
\gamma=\frac{(\mathrm{S} / \mathrm{N})_{\text {binary }}}{(\mathrm{S} / \mathrm{N})_{\text {isolated }}}
$$

where a pulsar in a binary system would undergo acceleration and a pulsar in an isolated system would have no acceleration. Figure 4.8 shows how $\gamma$ varies over orbital period and pulse period. 


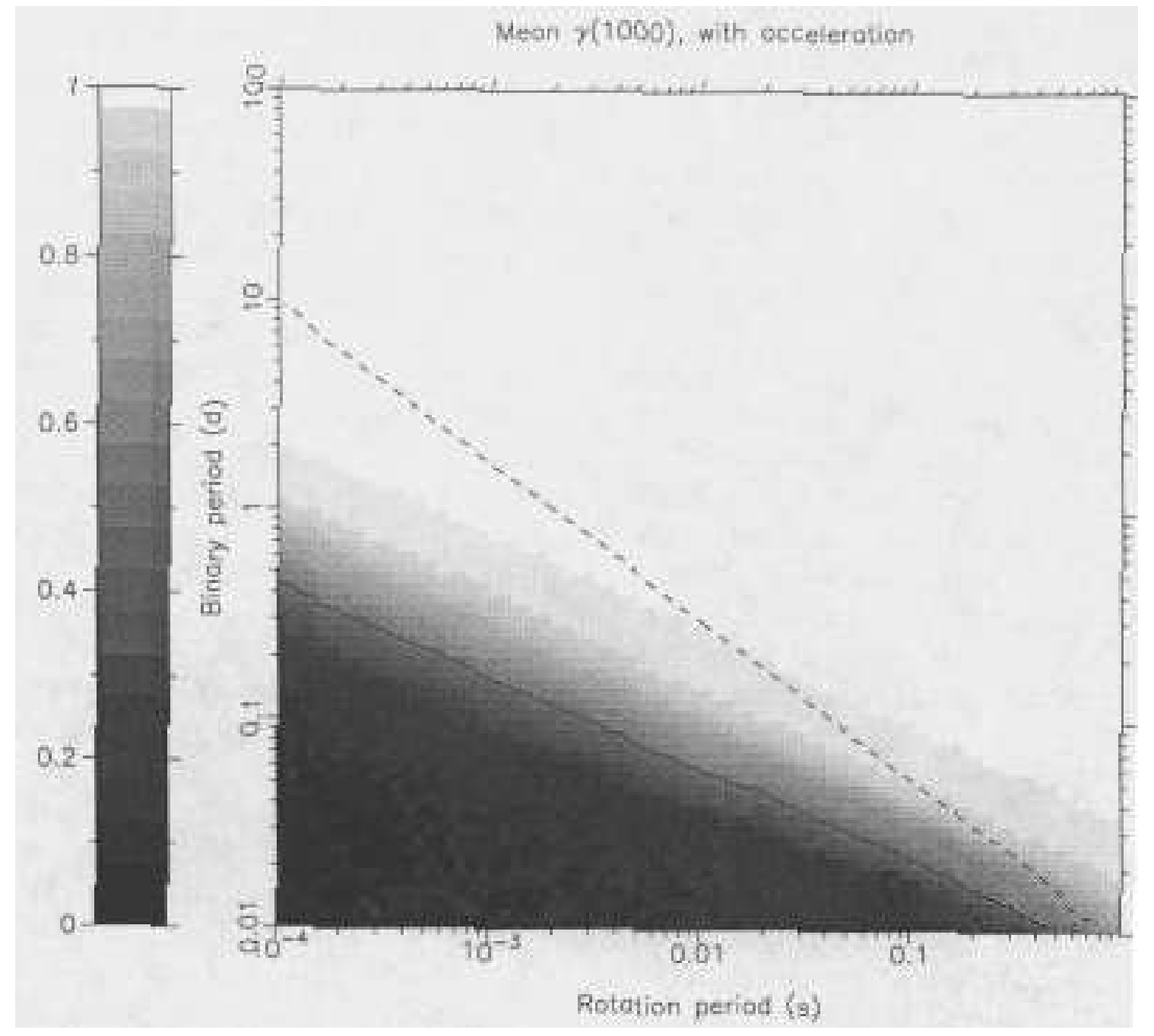

Figure 4.8: A gray-scale plot of $\gamma$ as a function of the orbital and spin periods when a pulsar is observed for integrations of $1000 \mathrm{~s}$. The dashed line shows the detection limit when there is no correction for acceleration, while the solid line shows the minimum detection limit for acceleration searches (Johnston \& Kulkarni, 1991). 


\section{Chapter 5}

\section{A Survey for Pulsars and Fast Transients in the Small Magellanic}

\section{Cloud}

\subsection{Introduction}

Containing five spin-powered radio pulsars and located at a distance of $60 \mathrm{kpc}$ (Harries et al., 2003), the Small Magellanic Cloud (SMC) holds the most distant pulsars ever detected. It has been surveyed multiple times for pulsars, most notably by McConnell et al. (1991), Crawford et al. (2001), and Manchester et al. (2006). These surveys have been successful in discovering new pulsars in both the Large Magellanic Cloud (LMC) and the SMC, however, they did not yield any new millisecond pulsars (MSPs). It is this lack of MSP detections and the opportunity to detect single, giant pulses that provide the motivation to undergo a new, high sensitivity search of the SMC.

The Berkeley-Parkes-Swinburne data recorder (BPSR) is a pulsar backend located at the Parkes Telescope in Parkes, Australia (Keith et al., 2010). It makes use of the $20 \mathrm{~cm}$ multibeam receiver (Staveley-Smith et al., 1996) and provides up to 4 times better time resolution and 30 times better frequency resolution over previous surveys of the Magellanic Clouds (e.g. Manchester et al., 2006). This greater sensitivity is particularly helpful for detecting MSPs and was the driving 


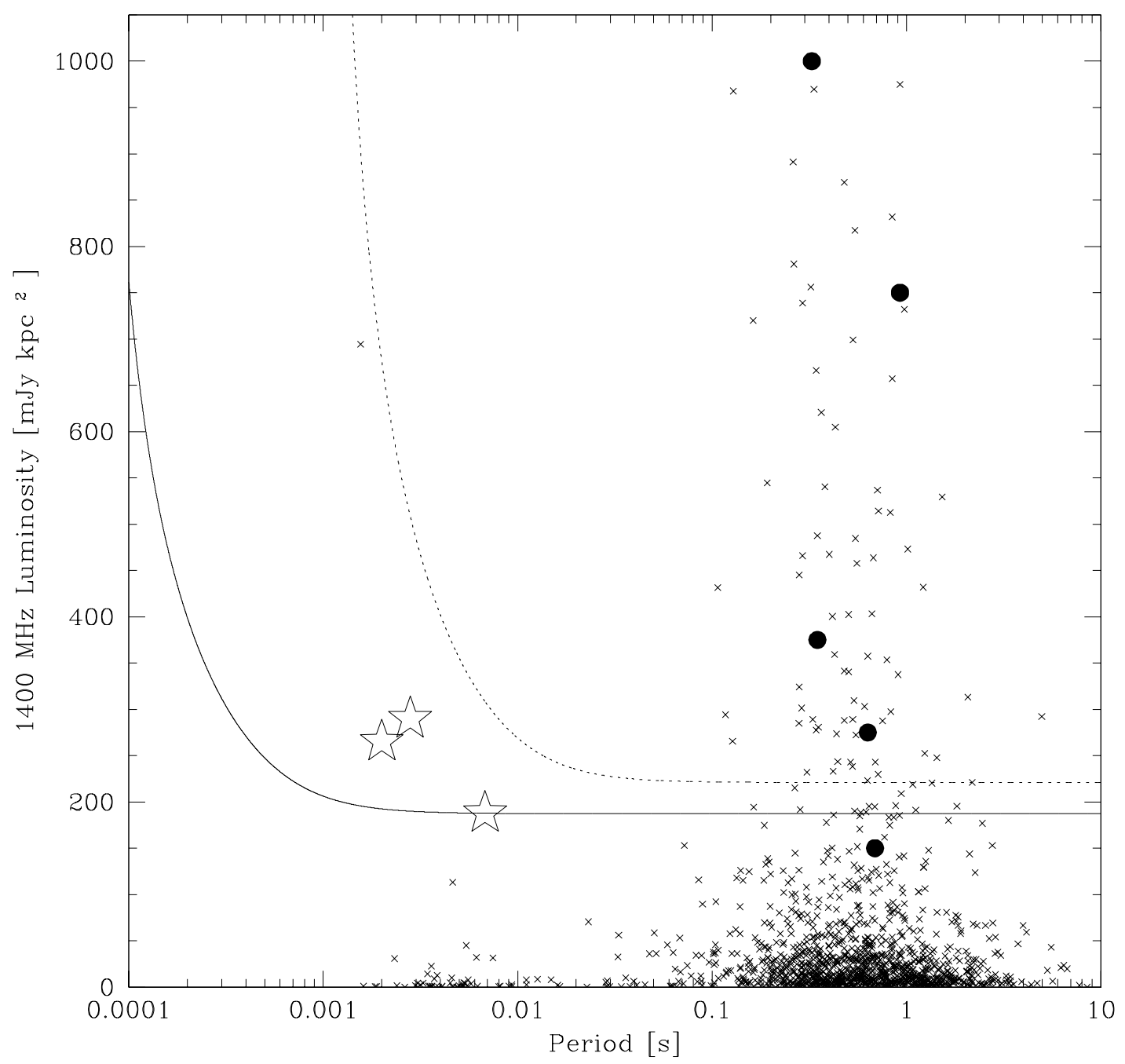

Figure 5.1: The minimum detectable luminosity of the Manchester et al. survey (dotted line) and the new BPSR survey (solid line). The luminosties of the Galactic pulsars are shown for reference. The solid dots represent the five known SMC pulsars, while the stars indicate the candidates from this survey. Note the significant improvement in sensitivity at short spin periods.

force for undergoing this new survey of the SMC. Figure 5.1 shows the improvement in sensitivity of this new survey over the last major survey of the Magellanic Clouds, done by Manchester et al. (2006). This survey was performed with the intent to search for bursting radio sources and to detect the first MSP in either the LMC or 
SMC.

\subsection{Observations}

Following the search procedures in Crawford et al. (2001), the central core of the SMC was searched between 2009 May and 2009 November using the BPSR pulsar backend. This survey made use of the Parkes $20 \mathrm{~cm}$ multibeam receiver (Staveley-Smith et al., 1996), which has 13 receivers, allowing it to effectively point at 13 different positions on the sky simultaneously. This survey consisted of 12 pointings, effectively covering the center of the SMC in 156 beams as shown in Figure 5.2. This covers approximately $6.7 \mathrm{deg}^{2}$ of the sky. Assuming that the SMC is located $60 \mathrm{kpc}$ from the Sun (Harries et al., 2003), this is equivalent to an area of $7.3 \mathrm{kpc}^{2}$.

The multibeam receiver operates at a center frequency of $1374 \mathrm{MHz}$, generally has a system noise temperature of $24 \mathrm{~K}$, and has a gain $(G)$ of $0.67 \mathrm{~K} / \mathrm{Jy}$. The BPSR backend has an available $400 \mathrm{MHz}$ bandwidth and can be broken into 1024 different frequency channels, each having a channel bandwidth of $0.390625 \mathrm{MHz}$. However, the actual bandwidth that is used for data processing is only $340 \mathrm{MHz}$, the native bandwidth of the multibeam receiver (Keith et al., 2010).

Each pointing was observed for a total of $8600 \mathrm{~s}$ and the data were sampled every $64 \mu \mathrm{s}$. Using the radiometer equation,

$$
S_{\text {min }}=\frac{\beta(\mathrm{S} / \mathrm{N})_{\min } \mathrm{T}_{\mathrm{sys}}}{G \sqrt{n_{p} t_{\mathrm{obs}} \Delta f}} \sqrt{\frac{W}{P-W}},
$$




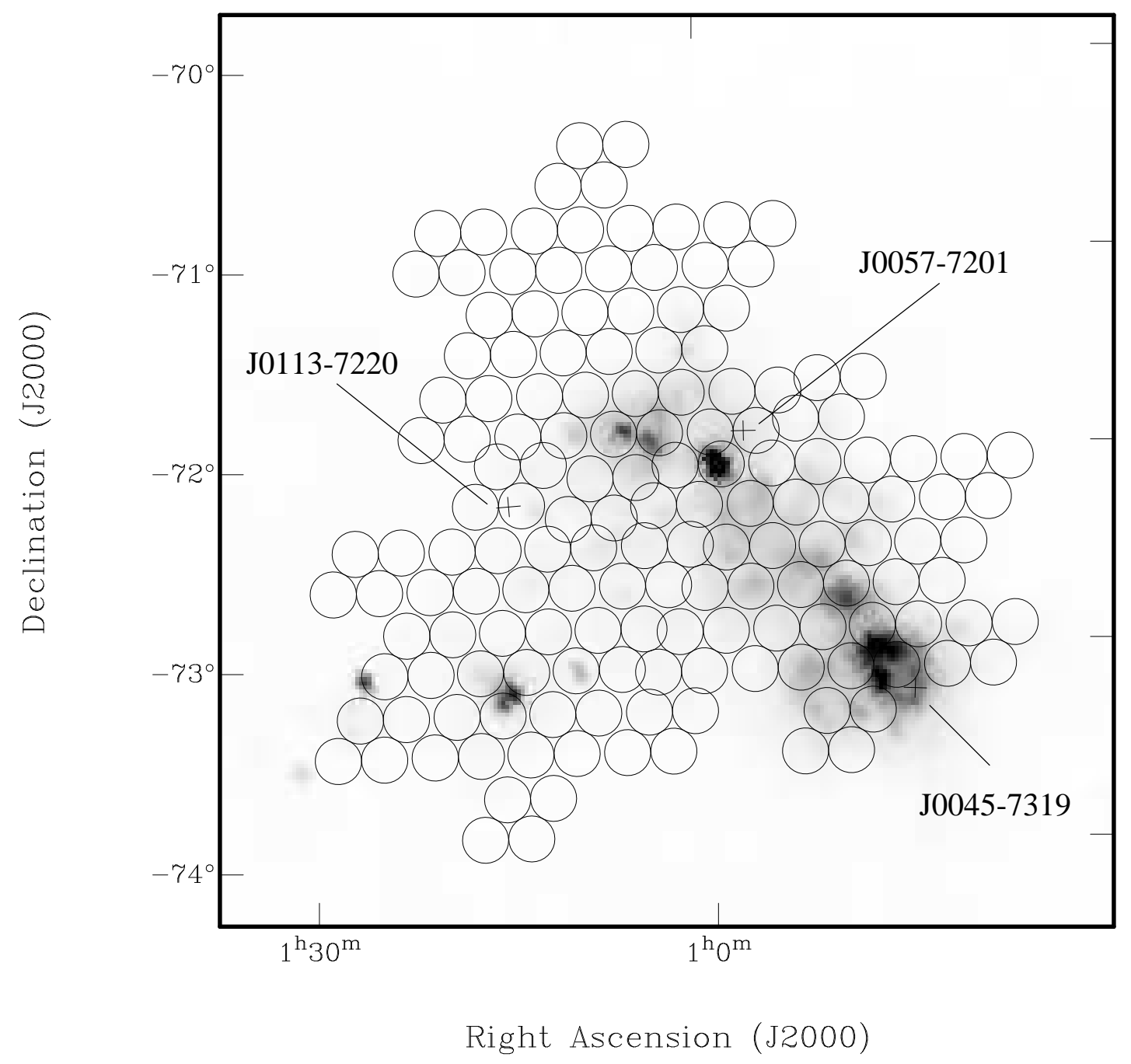

Figure 5.2: The central area of the SMC was searched using the multibeam receiver. This figure, originally from Crawford et al. (2001), shows the positions of each beam from all pointings on top of IRAS $60 \mu \mathrm{m}$ emission. The 3 pulsars plotted are from the Crawford et al. survey and were discovered in the direction of the SMC. 
yields a minimum flux density of $39.5 \mu \mathrm{Jy}$ for the observations. Values of $\beta \approx 1$, which is a correction factor, $n_{\mathrm{p}}=2$, which is the number of polarizations that were summed, and $W=0.1 P$ were assumed, and the minimum $\mathrm{S} / \mathrm{N}$ was 8 . The $1400 \mathrm{MHz}$ luminosity sensitivity for all pulsars is shown in Figure 5.1.

Data from each observation were archived on SDLT tapes and taken to West Virginia University for off-line processing. Using a 64-node Beowulf cluster, each beam was barycentered, de-dispersed, transformed using a Fast Fourier Transform, and then searched for periodic signals and single pulses using freely available software tools ${ }^{1}$ which implement the techniques described in Chapter 4. Dispersion measures

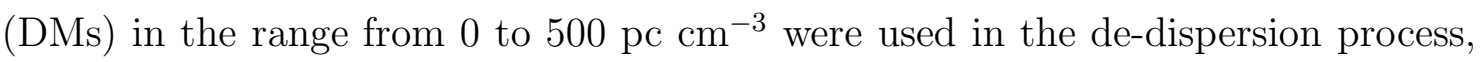
creating 959 trial DM values.

After the data underwent periodicity searches, the top candidates were filtered for further processing. Candidates were required to have a $\mathrm{S} / \mathrm{N}$ of at least 8 , a DM greater than $0 \mathrm{pc} \mathrm{cm}^{-3}$, and a period greater than $0.8 \mathrm{~ms}$. After filtering, roughly 60 candidates from each beam were folded and inspected by eye using the PRESTO ${ }^{2}$ processing suite to determine if additional observations were required.

In addition to periodicity searches, the data were also searched for single pulses. Using techniques discussed in Section 4.4, the data were searched for pulses ranging in width from $64 \mu \mathrm{s}$ to $65 \mathrm{~ms}$. A minimum flux density threshold for a single pulse

\footnotetext{
${ }^{1}$ http://sigproc.sourceforge.net

${ }^{2}$ http://github.com/scottransom/presto
} 
search can be calculated (see Cordes \& McLaughlin, 2003) as

$$
S_{\min }=\frac{(\mathrm{S} / \mathrm{N})_{\min } \mathrm{S}_{\mathrm{sys}}}{W} \sqrt{\frac{W}{n_{p} \Delta f}},
$$

where $(\mathrm{S} / \mathrm{N})_{\min }$ is the minimum $\mathrm{S} / \mathrm{N}, W$ is the maximum pulse width in seconds, $n_{p}$ is the number of polarizations, taken to be 2 here, and $\Delta f$ is the receiver bandwidth in Hz. $S_{\text {sys }}$ is the system sensitivity, which is $34 \mathrm{Jy}$ for the multibeam receiver. Assuming that $(\mathrm{S} / \mathrm{N})_{\min }=5$ and $\Delta f=340 \mathrm{MHz}$, the minimum flux density threshold for this survey is $30 \mathrm{mJy}$.

\subsection{Results}

Three known pulsars have been redetected in these observations. Two of these are located in the core of the SMC while the third lies in the Milky Way Galaxy along the line of sight of the SMC. Also, three pulsar candidates have appeared during this search of the SMC. These candidates would all be MSPs, making them virtually undetectable in previous surveys of the SMC. All redetections and candidates were seen using periodicity searches. Single pulse searches of the entire SMC yielded zero detections. The following sections describe these pulsars and candidates in more detail.

\subsubsection{Known Pulsars}

Three known pulsars were redetected in this survey. Plots of each redetection can be seen in Figures 5.3, 5.4, and 5.5. 
Table 5.1: A table of the 12 core pointings of the SMC, including observation time, MJD, RA/Dec, whether or not a known pulsar or pulsar candidate has been detected, and the minimum detectable flux density for a normal pulsar at $1400 \mathrm{MHz}$ within the center beam. Also included is a separate pointing (PSMC0099) centered on a known pulsar. The RA has units of hours, minutes, and seconds, and the DEC has units of degrees, arcminutes, and arcseconds.

\begin{tabular}{lrccccc}
\hline Pointing ID & $\mathrm{t}_{\text {obs }}(\mathrm{s})$ & MJD & RA (J2000) & DEC (J2000) & Pulsar? & $\mathrm{S}_{\text {min }}(\mathrm{mJy})$ \\
\hline PSMC0025a & 300 & 54968.978 & $00: 50: 31$ & $-72: 47: 39$ & no & 0.28 \\
PSMC0025b & 67 & 54969.030 & $00: 50: 31$ & $-72: 47: 39$ & no & 0.58 \\
PSMC0025c & 696 & 54969.190 & $00: 50: 31$ & $-72: 47: 39$ & no & 0.18 \\
PSMC0025d & 8619 & 54969.974 & $00: 50: 31$ & $-72: 47: 39$ & no & 0.04 \\
\hline PSMC0026 & 8622 & 54970.074 & $00: 53: 40$ & $-72: 48: 06$ & no & 0.04 \\
\hline PSMC0027 & 4423 & 54972.940 & $00: 52: 12$ & $-72: 35: 47$ & no & 0.07 \\
\hline PSMC0028a & 1268 & 54973.011 & $00: 49: 04$ & $-72: 35: 14$ & no & 0.13 \\
PSMC0028b & 3794 & 54973.172 & $00: 49: 04$ & $-72: 35: 14$ & no & 0.08 \\
\hline PSMC0033 & 2633 & 54974.087 & $01: 12: 54$ & $-73: 11: 13$ & yes & 0.09 \\
\hline PSMC0034 & 2211 & 54974.175 & $01: 16: 08$ & $-73: 10: 22$ & no & 0.10 \\
\hline PSMC0035 & 8461 & 55022.945 & $01: 14: 21$ & $-72: 58: 43$ & no & 0.04 \\
\hline PSMC0036a & 8622 & 55023.923 & $01: 11: 09$ & $-72: 59: 26$ & no & 0.04 \\
PSMC0036b & 8618 & 55148.506 & $01: 11: 09$ & $-72: 59: 26$ & no & 0.04 \\
PSMC0036c & 8617 & 55148.607 & $01: 11: 09$ & $-72: 59.26$ & no & 0.04 \\
\hline PSMC0037a & 252 & 55023.058 & $01: 05: 54$ & $-71: 35: 23$ & no & 0.30 \\
PSMC0037b & 8619 & 55224.194 & $01: 05: 54$ & $-71: 35: 23$ & no & 0.04 \\
\hline PSMC0038 & 8616 & 54977.814 & $01: 08: 51$ & $-71: 34: 59$ & yes & 0.04 \\
\hline PSMC0039 & 8620 & 54977.714 & $01: 07: 18$ & $-71: 23: 05$ & no & 0.04 \\
\hline PSMC0040a & 5224 & 55024.024 & $01: 04: 23$ & $-71: 23: 26$ & no & 0.07 \\
PSMC0040b & 8622 & 55150.441 & $01: 04: 23$ & $-71: 23: 26$ & no & 0.04 \\
\hline PSMC0099 & 7055 & 55150.543 & $01: 31: 28$ & $-73: 10: 09$ & yes & 0.06 \\
\hline
\end{tabular}




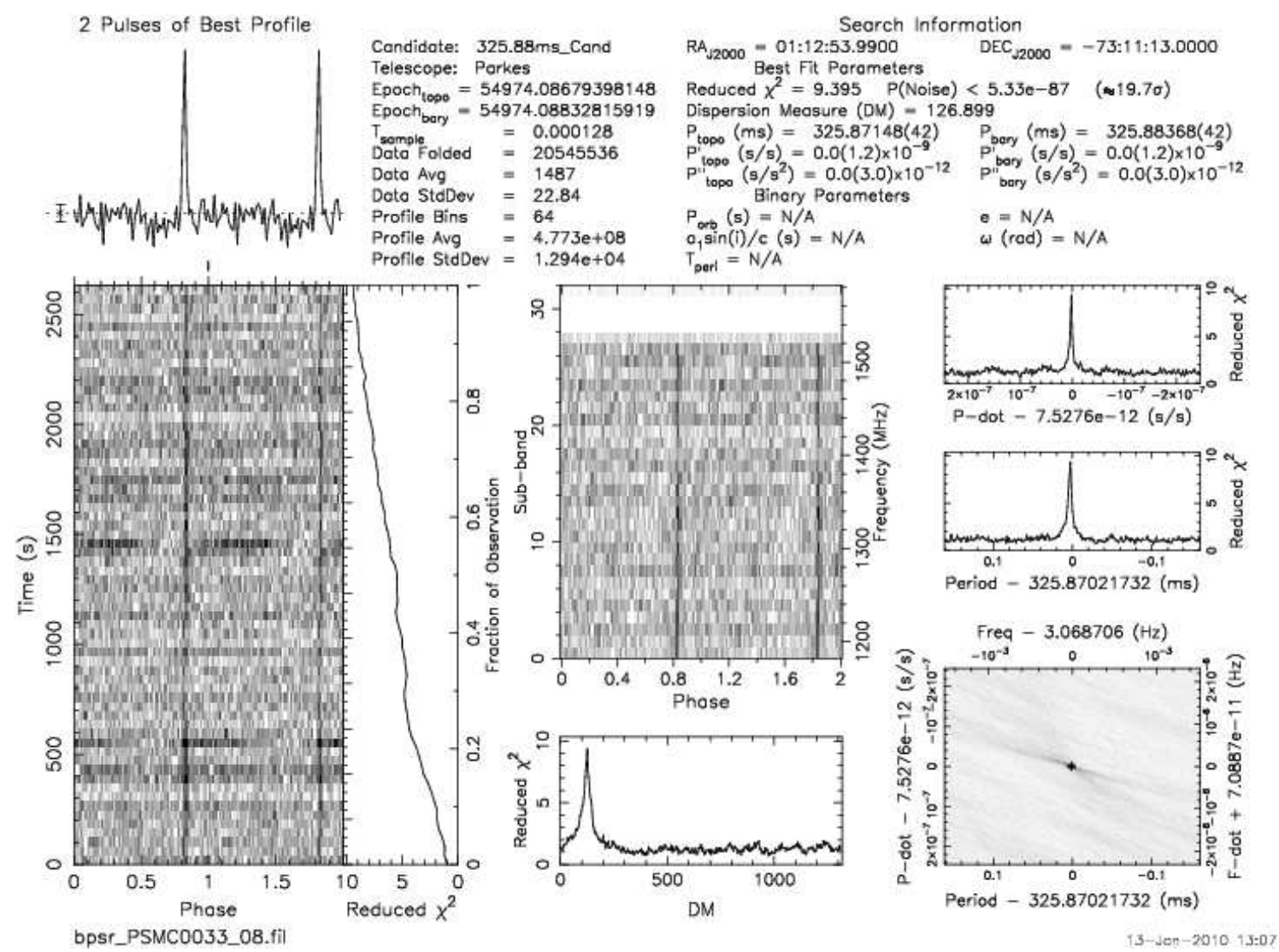

Figure 5.3: Search output showing the first redetected pulsar, J0113-7220.

\subsubsection{PSR J0113-7220}

The first pulsar redetected was J0113-7220, which was originally discovered in the Crawford et al. survey. With a period of $325.88 \mathrm{~ms}$ and a DM of $126.9 \mathrm{pc} \mathrm{cm}^{-3}$, this is a normal pulsar located in the SMC. This pulsar was observed in pointing PSMC0033 of this survey, which was only a partial pointing of 44 minutes, and had a $\mathrm{S} / \mathrm{N}$ of 20.6, as given by the seek pulsar search program ${ }^{3}$.

\footnotetext{
${ }^{3}$ http://sigproc.sourceforge.net
} 


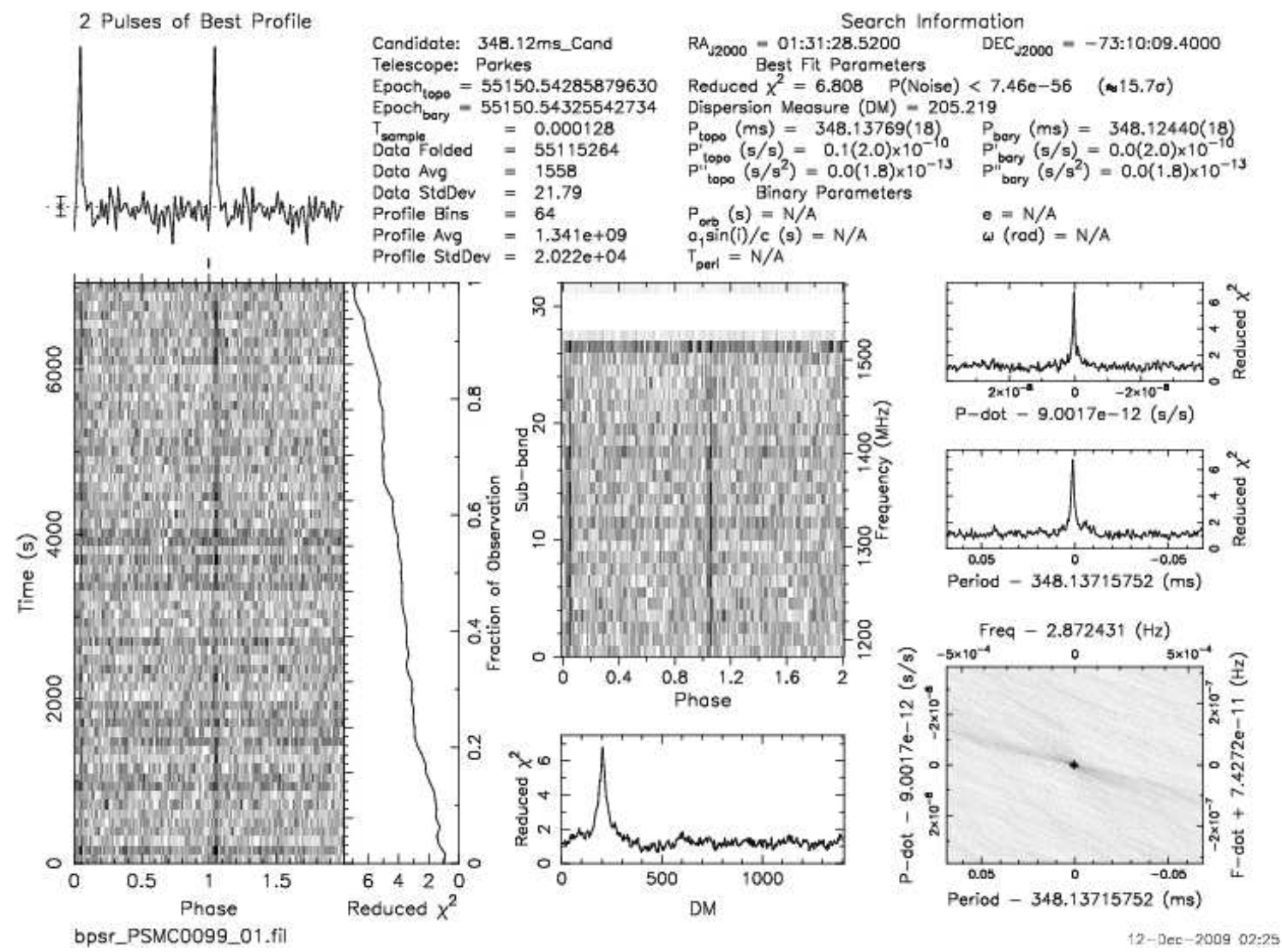

Figure 5.4: Search output showing the second redetected pulsar, J0131-7310.

\subsubsection{PSR J0131-7310}

Pulsar J0131-7310 was also redetected. Although not in the surveyed region, a separate pointing was performed centered on this pulsar. Originally discovered in the Manchester et al. survey, it has a period of $348.12 \mathrm{~ms}$ and a DM of $205.2 \mathrm{pc} \mathrm{cm}^{-3}$, making it another normal pulsar located within the estimated DM range (70-210) of the SMC. This pulsar was observed in pointing PSMC0099 with a S/N of 20.5. 


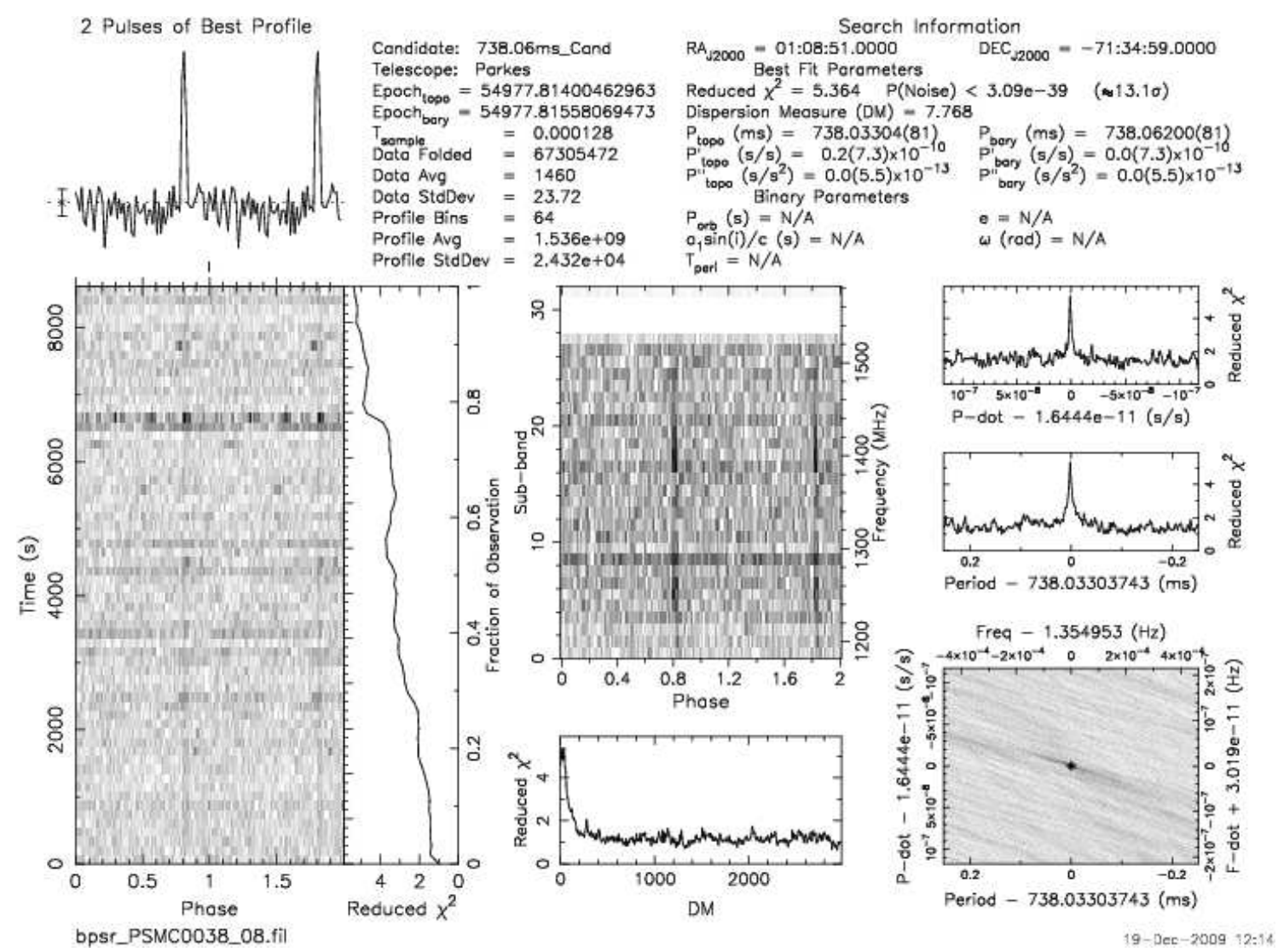

Figure 5.5: Search output showing the third redetected pulsar, J0057-7201.

\subsubsection{PSR J0057-7201}

The final pulsar redetected in this survey was J0057-7201, which was originally detected in the Crawford et al. survey. Having a period of $738.06 \mathrm{~ms}$ and a DM of $7.8 \mathrm{pc} \mathrm{cm}^{-3}$, this is a normal pulsar which lies in the direction of the SMC. The DM value differs significantly from the previously published value of $27 \mathrm{pc} \mathrm{cm}^{-3}$. The low DM and long period make it difficult to accurately determine the true DM, especially in just a single observation. The $\mathrm{S} / \mathrm{N}$ is most likely a very high value for a large range of DMs. The low DM, however, means that the pulsar is located within the Milky Way Galaxy. This pulsar was observed in pointing PSMC0038, which was a full-length pointing, and had a $\mathrm{S} / \mathrm{N}$ of 9.4. 


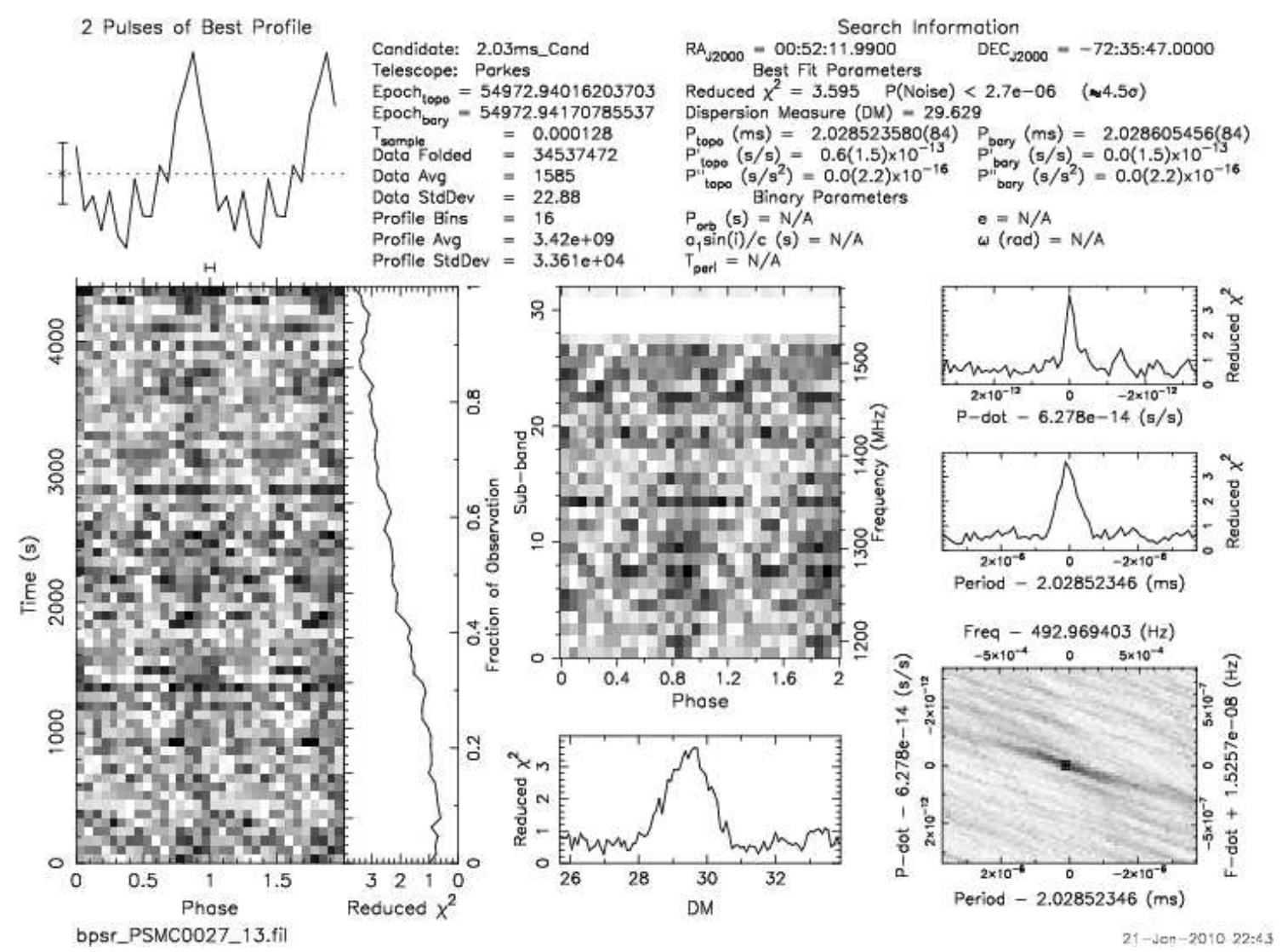

Figure 5.6: Search output showing MSP candidate SMC J0042-7300.

\subsubsection{MSP Candidates}

This survey of the SMC also produced multiple MSP candidates.

\subsubsection{SMC J0042-7300}

The first MSP candidate is located at an RA of $00 \mathrm{~h} 42 \mathrm{~m} 10 \mathrm{~s}$ and a DEC of $-73^{\circ} 00^{\prime} 16^{\prime \prime}$, and has a period of $2.03 \mathrm{~ms}$ and a DM of $29.6 \mathrm{pc} \mathrm{cm}^{-3}$. This makes it a possible foreground pulsar. This was observed in pointing PSMC0027, which was 74 minutes in length, and had a $\mathrm{S} / \mathrm{N}$ of 8.2. This yields a flux density of $0.07 \mathrm{mJy}$ and a $1400 \mathrm{MHz}$ luminosity of $265 \mathrm{mJy} \mathrm{kpc}^{2}$ using the assumed distance to the SMC of $60 \mathrm{kpc}$. Figure 5.6 shows the original detection. 


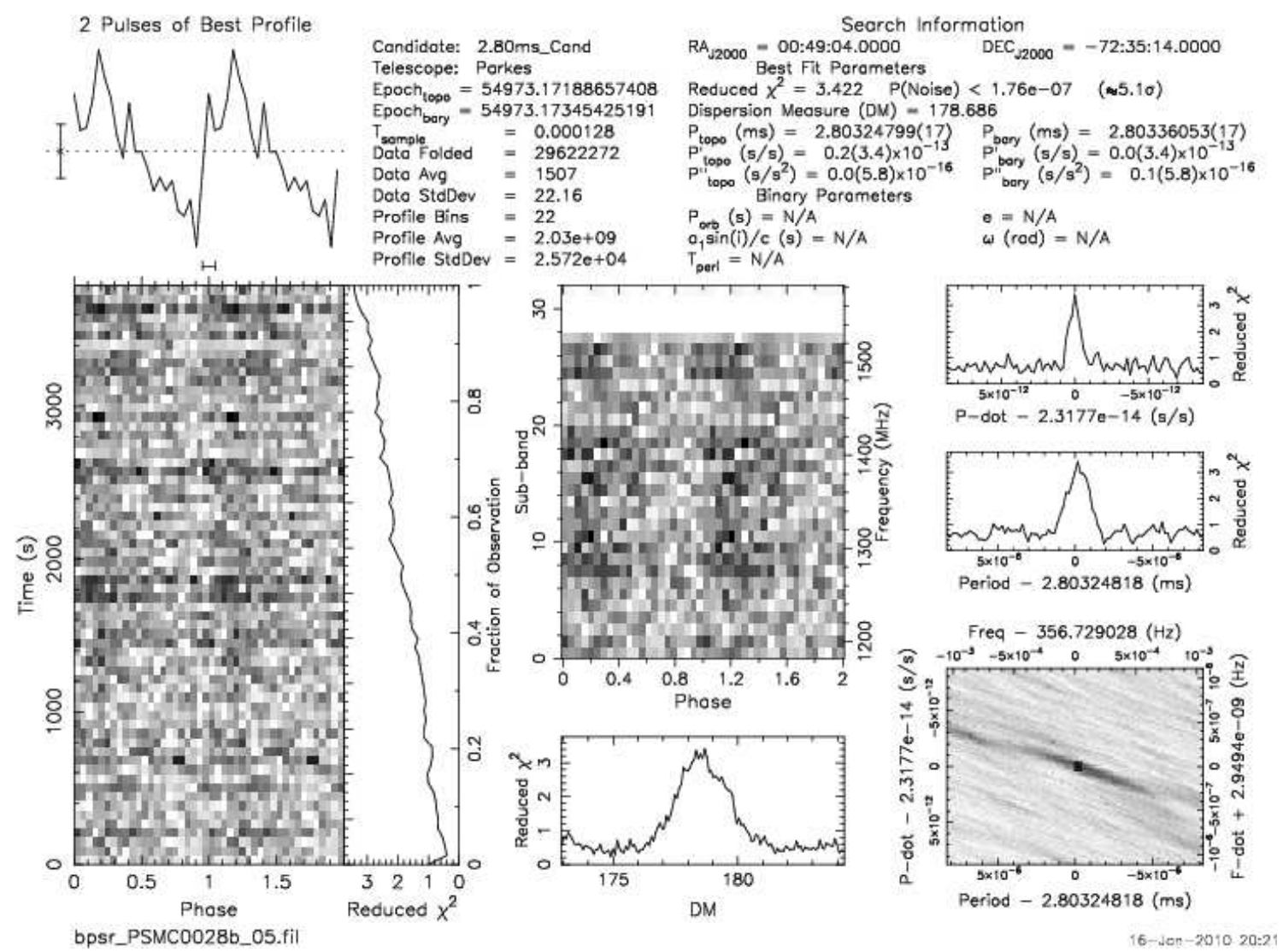

Figure 5.7: Search output showing MSP candidate SMC J0045-7300. Note that there appears to be a scattering tail in the pulse profile (see Section 1.2.7).

\subsubsection{SMC J0045-7300}

The second MSP candidate is located at an RA of 00h $45 \mathrm{~m} 45 \mathrm{~s}$ and a DEC of $-73^{\circ} 00^{\prime} 20^{\prime \prime}$, has a period of $2.80 \mathrm{~ms}$, and a DM of $178.7 \mathrm{pc} \mathrm{cm}^{-3}$. This DM places this candidate within the SMC. Observed in pointing PSMC0028b for slightly more than 1 hour, the original detection can be seen in Figure 5.7. It had a $\mathrm{S} / \mathrm{N}$ of 8.3, which translates to a flux density of $0.08 \mathrm{mJy}$ and a $1400 \mathrm{MHz}$ luminosity of $290 \mathrm{mJy} \mathrm{kpc}^{2}$. 


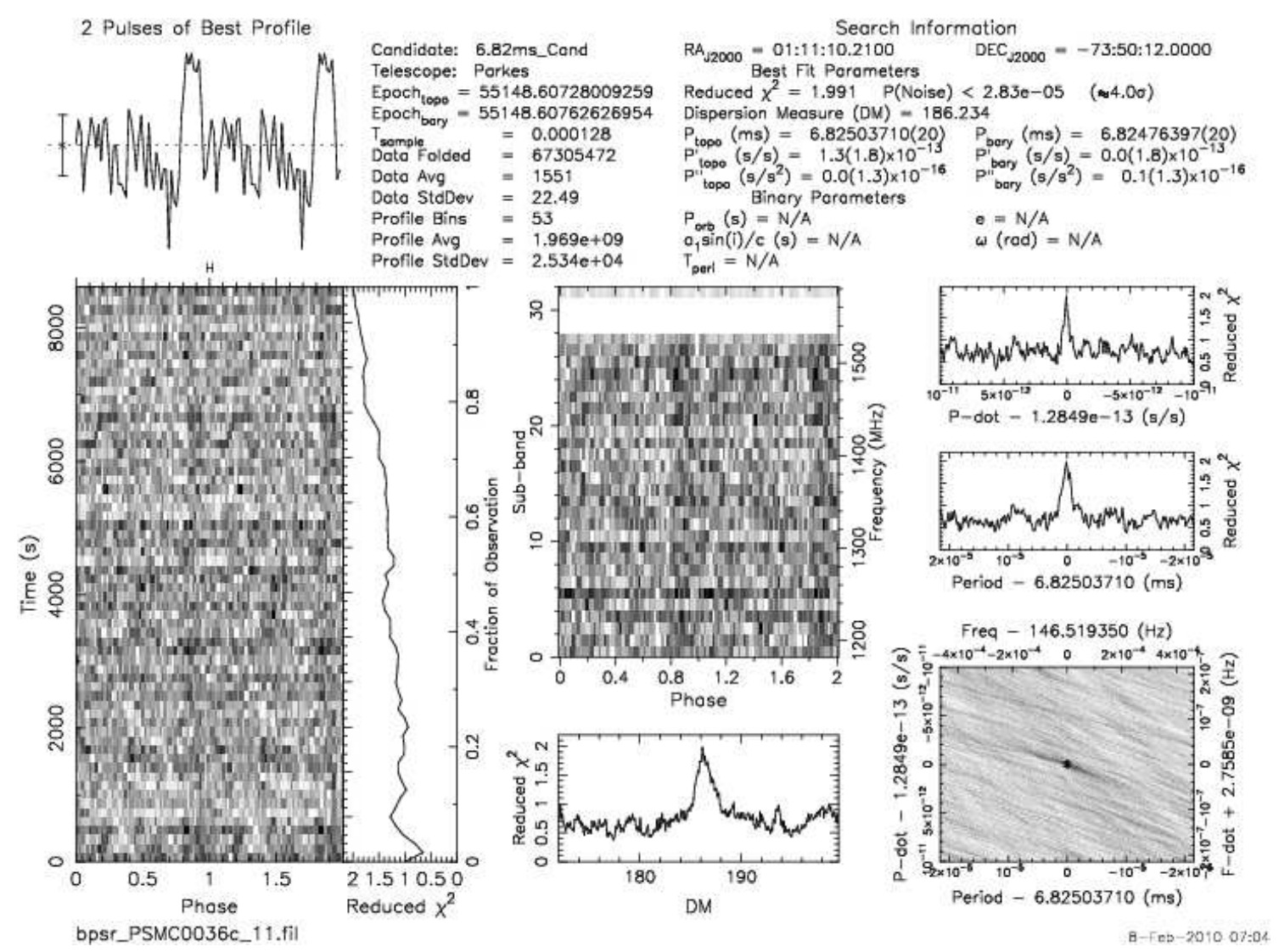

Figure 5.8: Search output showing MSP candidate SMC J0111-7350.

\subsubsection{SMC J0111-7350}

The final MSP candidate is located at an RA of $01 \mathrm{~h} 11 \mathrm{~m} 10 \mathrm{~s}$ and a DEC of $-73^{\circ} 50$ '12", has a period of $6.82 \mathrm{~ms}$, and a DM of $186.2 \mathrm{pc} \mathrm{cm}^{-3}$. Again, this DM places it within the SMC. It had a $\mathrm{S} / \mathrm{N}$ of 8.1, and a plot from pointing PSMC0036c can be found in Figure 5.8. The flux density was found to be $0.05 \mathrm{mJy}$, giving it a $1400 \mathrm{MHz}$ luminosity of $188 \mathrm{mJy} \mathrm{kpc}^{2}$.

\subsubsection{Follow-up Observations}

Each of the three MSP candidates will be observed a second time with a full length pointing. The center beam of the multibeam receiver will be centered directly 
on each candidate to allow for the highest sensitivity possible. These observations, to be carried out in the first few months of 2011, will be able to either confirm or reject these candidates as being actual pulsars.

\subsection{Discussion}

\subsubsection{Candidates Versus Known Pulsars}

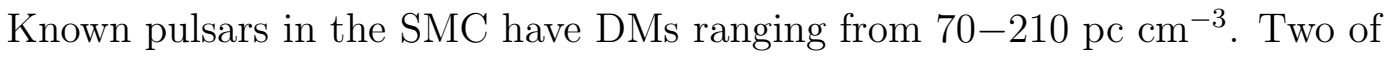
the candidates produced from this survey fall within that range. The third has a much lower DM, likely making it a foreground pulsar.

All three of the candidates have spin periods on the order of a few milliseconds. Since all of the currently known SMC pulsars fall into the normal pulsar category, these candidates are especially unique. They would be the first MSPs discovered within either of the Magellanic Clouds.

\subsubsection{Expected S/N}

Using the catalog flux density of the known SMC radio pulsars and the radiometer equation, the expected $\mathrm{S} / \mathrm{N}$ can be calculated. Assuming that each pulsar was observed with a full pointing, these values are shown in Table 5.2 along with other pulsar properties, including spin period, DM, and $1400 \mathrm{MHz}$ flux density. The expected $\mathrm{S} / \mathrm{N}$ for each pulsar appears to be reasonable, especially considering that the observed $\mathrm{S} / \mathrm{N}$ for the 2 observed SMC pulsars are both within $15 \%$ of the expected values. 
Since J0113 was not observed with the center beam of the multibeam receiver, the telescope gain was slightly lower $(0.581 \mathrm{~K} / \mathrm{Jy})$. The values for the gains from each beam can be found in Manchester et al. (2001). In addition, the pulsar was not located at the center of the telescope beam, meaning that the observed flux was slightly lower than it would be had the telescope been pointed directly at it (similar to how pointing PSMC0099 pointed directly at J0131). To account for this, the distance from the pulsar to the center of beam, $r$, was measured, and then a flux degradation factor, $\delta_{\text {beam }}$, was calculated by

$$
\delta_{\text {beam }}=\exp \left(\frac{-r^{2}}{f_{\text {beam }}^{2}}\right) \text {, }
$$

where $f_{\text {beam }}=b /(2 \sqrt{\ln 2})$ for a telescope beamwidth of $b$ degrees (Lorimer et al., 1993). This model assumes that the telescope beam has a Gaussian power pattern.

\subsubsection{Single Pulses}

As mentioned earlier, there were no single pulses observed in the direction of the SMC. A few of the reasons for the lack of detections will be discussed here.

First, the SMC is located nearly $60 \mathrm{kpc}$ away from the Earth. This means that the observed flux density from an average pulse of the pulsar will be much lower than other pulsars (with previously observed single pulses) located within the Milky Way. It is possible that the flux density of the pulses arriving at the Earth is too weak to be observed using the parameters of this survey.

A related issue is that of giant pulses. A few pulsars (e.g. the Crab Pulsar) 
Table 5.2: A table showing the Period, DM, $1400 \mathrm{MHz}$ flux density, and $r$ values of the 5 currently known SMC radio pulsars and the redetected foreground pulsar. Values for the SMC pulsars were obtained from Manchester et al. (2006), while the foreground pulsar values came from Crawford et al. (2001). Also included are the observed and expected $\mathrm{S} / \mathrm{N}$ values from this survey. The first two pulsars were not redetected because they did not lie within a FWHM (full width half maximum) of any of the beams from this survey, so their expected $\mathrm{S} / \mathrm{N}$ values were calculated assuming that they were observed with a full-length pointing using the center beam of the multibeam receiver and an N/A was placed in the $r$ column. J0111-7131 was within beam 8 of PSMC0038, however, due to its low flux density, it was not detected.

\begin{tabular}{lcccccc}
\hline JName & $P(\mathrm{~s})$ & $\begin{array}{c}\text { Catalog DM } \\
\left(\mathrm{cm} \mathrm{pc}^{-3}\right)\end{array}$ & $\begin{array}{c}S_{1400} \\
(\mathrm{mJy})\end{array}$ & $\begin{array}{c}\text { Observed } \\
\mathrm{S} / \mathrm{N}\end{array}$ & $\begin{array}{c}\text { Expected } \\
\mathrm{S} / \mathrm{N}\end{array}$ & $\begin{array}{c}r \\
(\operatorname{arcmin})\end{array}$ \\
\hline J0045-7042 & 0.6323 & 70.0 & 0.11 & $\mathrm{~N} / \mathrm{A}$ & 17.1 & $\mathrm{~N} / \mathrm{A}$ \\
$\mathrm{J} 0045-7319$ & 0.9262 & 105.4 & 0.30 & $\mathrm{~N} / \mathrm{A}$ & 46.6 & $\mathrm{~N} / \mathrm{A}$ \\
$\mathrm{J} 0111-7131$ & 0.6885 & 76.0 & 0.06 & N/A & 6.9 & 4.1 \\
$\mathrm{~J} 0113-7220$ & 0.3258 & 125.4 & 0.40 & 20.6 & 23.6 & 1.9 \\
J0131-7310 & 0.3481 & 205.2 & 0.15 & 20.5 & 19.3 & $<0.5$ \\
\hline J0057-7201 & 0.7381 & 27.0 & N/A & 9.4 & N/A & 0.6 \\
\hline
\end{tabular}

are known to periodically emit pulses that are 10s-100s of times stronger than their average pulses. If none of the SMC pulsars (or candidates) exhibit properties such as the Crab Pulsar, then these bright giant pulses would not be detected in this survey.

Finally, radio frequency interference $(\mathrm{RFI})$ is also a possible explanation for the lack of single pulse detections. As evidenced in the previous chapter, RFI removal effectively involves completely zapping signals that appear to have originated on the Earth, or equivalently at a DM of $0 \mathrm{pc} \mathrm{cm}^{-3}$. Single pulses that may be bright enough to be detected could potentially be erased when the RFI is removed.

The lack of single pulse detections leads directly to a Bayesian analysis of the 
underlying population of bursting sources. Following methods discussed in Gregory (2005) and Wall \& Jenkins (2001), a probability density function (PDF) of the number of bursting sources can be calculated. First, it is assumed that the rate of bursts above a minimum flux density, $S$, can be represented as

$$
R(>S)=K S^{\alpha}
$$

where $K$ is a constant and $\alpha$ is the spectral index. The predicted number of pulses observed from a population of $N$ sources in an observation of length $T$ above a minimum flux, $S_{\min }$, is then just

$$
n_{\text {pulses }}=K N S_{\min }^{\alpha} T
$$

Assuming the pulses follow Poissonian statistics, the probability of observing $m$ pulses given the model parameters $N, \alpha$, and $K$ can be written as

$$
p(m \mid N, \alpha, K)=\frac{n_{\text {pulses }}^{m} \exp \left(-n_{\text {pulses }}\right)}{m !}
$$

where the $\mid$ symbol denotes a conditional probability. Bayes' theorem may now be applied to find the joint posterior PDF for $N, \alpha$, and $K$ if $m$ pulses are detected. Neglecting normalizing factors, the joint posterior is simply

$$
p(N, \alpha, K \mid m) \propto p(m \mid N, \alpha, K) p(N, \alpha, K) .
$$


Here, the first term on the right-hand side of this expression is the likelihood factor and is given by our Poissonian expression above. The second term is the joint prior PDF for $N, \alpha$ and $K$. For this case, where there are no detections, $m=0$. Taking the most conservative approach and assuming uniform (i.e. non-informative) priors on the model parameters, the desired posterior PDF for $N$ can be expressed by marginalizing over $\alpha$ and $K$. In this way, it is found that

$$
p(N \mid m=0) \propto \int_{\alpha_{\min }}^{\alpha_{\max }} \int_{K_{\min }}^{K_{\max }} \exp \left(-n_{\text {pulses }}\right) d \alpha d K,
$$

where $K_{\min }$ and $K_{\max }$ are the ranges considered for $K$, and similarly for $\alpha_{\min }$ and $\alpha_{\max }$. To get plausible ranges for $K$ and $\alpha$, the only known giant pulsing emitter in the LMC known to date, PSR B0540-69 (Johnston \& Romani, 2003), is considered, and from their Figures 2 and 3 it is found that $K_{\min }=0.01, K_{\max }=0.02$, $\alpha_{\min }=-2.0$, and $\alpha_{\max }=-1.0$. The above double integral is evaluated numerically assuming $S_{\min }=30 \mathrm{mJy}$ and $T=8600 \mathrm{~s}$, and the resulting distribution is shown as a histogram in Figure 5.9. From this distribution there is a $95 \%$ likelihood that there are no more than 15 bursting sources (similar to B0540-69) in the SMC.

\subsubsection{Survey Limitations}

Aside from telescope limitations such as size, receiver temperature, and observing frequency, the biggest limitation is observation time. According to the radiometer equation, the minimum observable flux density is directly affected by the length of the observation. The longer the observation, the lower the minimum flux 


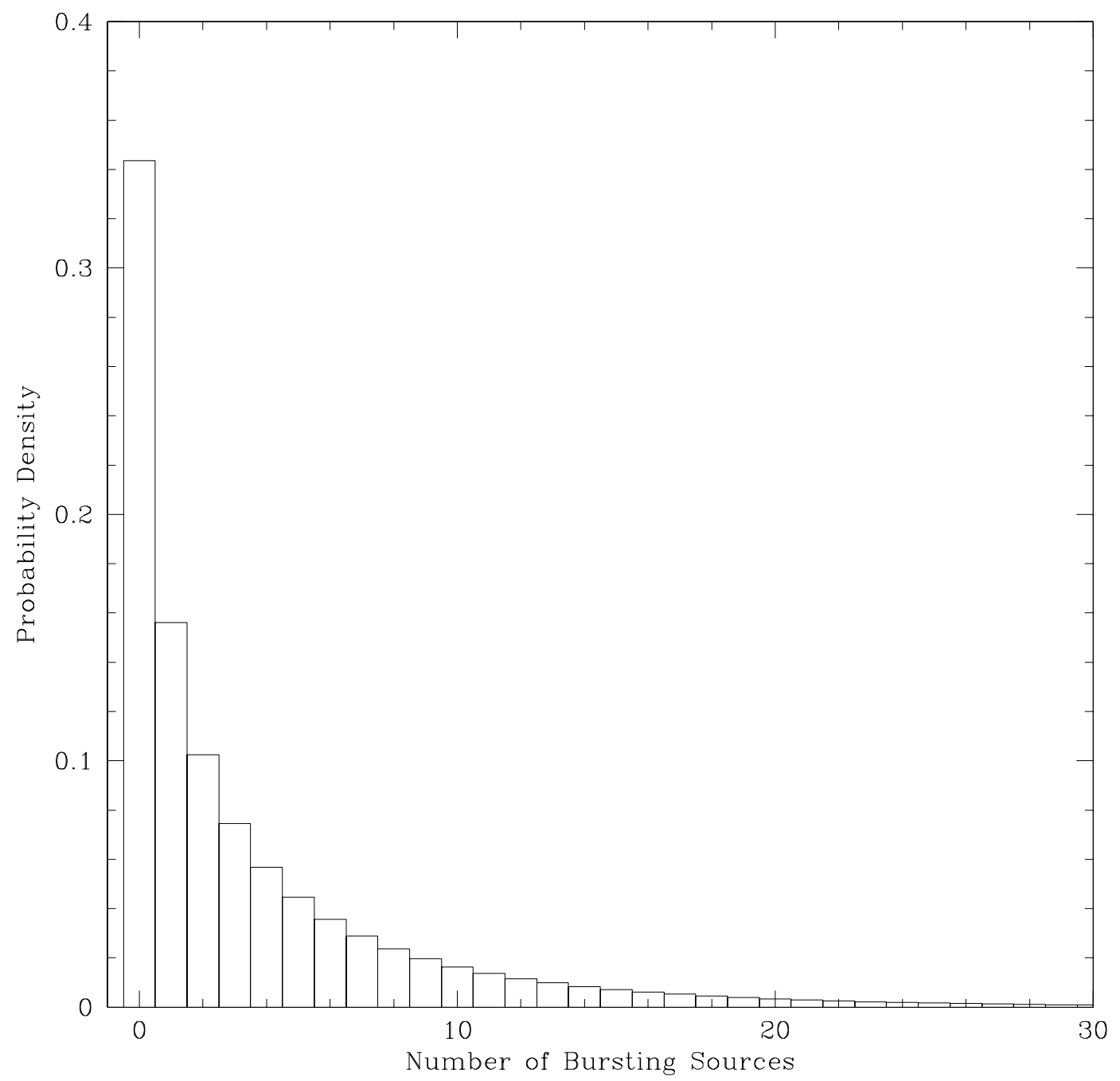

Figure 5.9: The probability density function of having a given number of bursting sources when no pulses were observed in the SMC. The $95 \%$ upper limit lies at 15 sources. 
density becomes.

For this survey, an observation time of nearly 2.5 hours was used. Since the $\mathrm{SMC}$ is not a circumpolar source when viewed from the Parkes Observatory, there is a maximum continuous observation time. Realistically, this maximum time would be approximately 5 hours. Since this only reduces $S_{\min }$ to $27.3 \mu \mathrm{Jy}$ from $39.5 \mu \mathrm{Jy}$, this survey would not be significantly more sensitive if this maximum integration time was used.

Another limitation is the acceleration search. To be sensitive to MSPs with periods on the order of $2 \mathrm{~ms}$ using an observation time of nearly $9000 \mathrm{~s}$, the acceleration step size, $\Delta a=c P / T^{2}$, is approximately $0.007 \mathrm{~m} \mathrm{~s}^{-2}$. Using steps of $0.02 \mathrm{~m} \mathrm{~s}^{-2}$, searching accelerations up to $\pm 10 \mathrm{~m} \mathrm{~s}^{-2}$ would require 1000 additional searches at each DM, effectively increasing the processing time by a factor of 1000 . However, when this search is actually carried out, a smaller range of accelerations (possibly out to $\pm 3 \mathrm{~m} \mathrm{~s}^{-2}$ ) and a specific DM range $\left(70-210 \mathrm{pc} \mathrm{cm}^{-3}\right.$ ) will be used so that the actual increase in processing time will be around a factor of 4 or 5 .

Other limitations of this survey, many relating to hardware and computer issues, would make this survey more sensitive to sub-millisecond pulsars. As no sub-millisecond pulsars have currently been detected, this would be an exciting class of pulsars to discover and study. 


\subsection{Conclusions}

This survey has successfully redetected three SMC pulsars. A fourth known SMC pulsar is located outside the bounds of this survey, and a fifth was too weak to be observed in only a partial pointing.

Three new candidates have appeared from processing the data of this survey. Two of them are located within the SMC, while the third lies in the foreground along the line of sight. All of these candidates have periods in the millisecond range, which would make them the first MSPs in the Magellanic Clouds. Based on the simulations of Ridley \& Lorimer (2010), also seen in Chapter 3, an upper limit of 8,000 MSPs can now be placed on the SMC.

After successfully completing this survey of the SMC, the logical plan is to extend the survey to cover the LMC. Currently, the central core of the LMC is being surveyed using the same setup as the SMC survey discussed here. If the central LMC survey yields promising results, then observations of the outer regions of the LMC will be planned. In addition to surveying the LMC, acceleration searches will be performed on the SMC survey data in an attempt to discover pulsars that are in binary systems. 


\section{Chapter 6}

\section{Conclusions}

This work has introduced pulsars, described their properties, modeled their population distributions, explained in detail how to search for them, and presented the results of a survey of the Small Magellanic Cloud.

Chapter 1 began by laying the foundation of the fundamental pulsar properties, from spin period to flux density to magnetic field. Specific attention was made to distinguish between the two major types of pulsars, known as millisecond pulsars (MSPs) and normal pulsars.

Chapter 2 took these properties and modeled the pulsar population within the Milky Way Galaxy by performing various Monte Carlo computer simulations. These simulations were able to recreate the observed pulsar population and thus create a working model of pulsars that can be further studied for purposes of learning more about individual or groups of pulsars, or even predicting how many pulsars should be detected in a survey of the Galaxy. Specifically, there were three major parameters that were constrained here. Magnetic alignment angles should have a random initial distribution, pulsar luminosities should have a $P$ and $\dot{P}$ dependence, and braking indices, $n$, do not significantly affect the overall pulsar population.

Chapter 3 expanded on the simulations presented in Chapter 2 and carried out a similar simulation of the Large and Small Magellanic Clouds (LMC and SMC). The 
pulsar populations generated by these simulations were able to accurately reproduce the results of previous surveys of the Magellanic Clouds. Upper limits of 15,000 and 23,000 MSPs have been placed on the LMC and SMC, respectively. Taking these models a step further, predictions were made regarding the potential pulsar detections in a future survey of both the LMC and SMC.

Chapter 4 discussed the three major types of pulsar searches used today. Periodicity searches are effective at discovering the repeated signals that pulsars emit, and sensitive to a wide range of periods. Single pulse searches can find pulses whose intensity is significantly greater than the underlying noise. Some otherwise weak pulsars have been discovered only due to these occasionally bright pulses that they emit. Finally, acceleration searches are extremely useful when searching for pulsars in binary orbits. By accounting for a range of possible accelerations, pulsars can be detected even though their motion is oftentimes largely perturbed.

Finally, Chapter 5 presented the initial results of a survey of the Small Magellanic Cloud, performed in 2009. The survey redetected three previously known pulsars (two in the SMC and one lying along the line of sight toward the SMC) and yielded three potential MSPs. Further observations are being done on these candidates with the hopes of confirming one or more of them as pulsars.

There are many areas for future work to be done. The pulsar parameter analysis performed in Chapter 2 can be adapted to study even more parameters and can even focus on covariances of multiple parameters. Extending the SMC survey of Chapter 5 to the LMC will certainly redetect known LMC pulsars and should uncover even more MSP candidates. Also, a fully dynamical model of both the LMC 
and SMC could be created, similar to the work in Chapter 2 done with the Milky Way Galaxy.

There are also telescope improvements that will allow for further studies to be done. The Square Kilometre Array will be up to an order of magnitude more sensitive than current telescopes and this will allow for even more pulsars to be detected while using a smaller amount of observing time. With surveys being completed faster, more surveys can be performed which enables more pulsars to be detected and studied. These new pulsars can help to understand individual groups of pulsars, such the young pulsars, the MSPs, and ones with low luminosities. Finding additional pulsars in the Magellanic Clouds will also help compare that population to that of the Galaxy. 


\section{Bibliography}

Arzoumanian Z., Chernoff D. F., Cordes J. M., 2002, ApJ, 568, 289

Baade W., Zwicky F., 1934, Proc. Nat. Acad. Sci., 20, 254

Bekki K., Stanimirović S., 2009, MNRAS, 395, 342

Bhattacharya D., van den Heuvel E. P. J., 1991, Phys. Rep., 203, 1

Bhattacharya D., Wijers R. A. M. J., Hartman J. W., Verbunt F., 1992, A\&A, 254, 198

Biggs J. D., 1990, MNRAS, 245, 514

Bracewell R., 1999, The Fourier Transform and its Applications. McGraw-Hill, New York

Camilo F., Lorimer D. R., Freire P., Lyne A. G., Manchester R. N., 2000, ApJ, 535, 975

Carlberg R. G., Innanen K. A., 1987, AJ, 94, 666

Chen K., Ruderman M., 1993, ApJ, 408, 179

Contopoulos I., Spitkovsky A., 2006, ApJ, 643, 1139

Cordes J. M., Lazio T. J. W., 2002, NE2001. I. A New Model for the Galactic Distribution of Free Electrons and its Fluctuations, astro-ph/0207156

Cordes J. M., McLaughlin M. A., 2003, ApJ, 596, 1142

Crawford F., Kaspi V. M., Manchester R. N., Lyne A. G., Camilo F., D'Amico N., 2001, ApJ, 553, 367

Deneva J. S., Cordes J. M., McLaughlin M. A., Nice D. J., Lorimer D. R., Crawford F., Bhat N. D. R., Camilo F., Champion D. J., Freire P. C. C., Edel S., Kondratiev V. I., Hessels J. W. T., Jenet F. A., Kasian L., Kaspi V. M., Kramer M., Lazarus P., Ransom S. M., Stairs I. H., Stappers B. W., van Leeuwen J., Brazier A., Venkataraman A., Zollweg J. A., Bogdanov S., 2009, ApJ, 703, 2259

Duncan R. C., Thompson C., 1992, ApJ, 392, L9

DuPlain R., Ransom S., Demorest P., Brandt P., Ford J., Shelton A. L., 2008, in Society of Photo-Optical Instrumentation Engineers (SPIE) Conference Series Vol. 7019 of Presented at the Society of Photo-Optical Instrumentation Engineers (SPIE) Conference, Launching GUPPI: the Green Bank Ultimate Pulsar Processing Instrument

Eatough R. P., Keane E. F., Lyne A. G., 2009, MNRAS, 395, 410 
Edwards R. T., Bailes M., van Straten W., Britton M. C., 2001, MNRAS, 326, 358

Faucher-Giguère C.-A., Kaspi V. M., 2006, ApJ, 643, 332

Gold T., 1968, Nature, 218, 731

Gonidakis I., Livanou E., Kontizas E., Klein U., Kontizas M., Belcheva M., Tsalmantza P., Karampelas A., 2009, A\&A, 496, 375

Gonthier P. L., Ouellette M. S., Berrier J., O’Brien S., Harding A. K., 2002, ApJ, 565,482

Gotthelf E. V., Vasisht G., Boylan-Kolchin M., Torii K., 2000, ApJ, 542, L37

Green D. A., Gull S. F., 1984, Nature, 312, 527

Gregory P., 2005, Bayesian logical data analysis for the Physical Sciences. Cambridge University Press

Gunn J. E., Ostriker J. P., 1970, ApJ, 160, 979

Harries T. J., Hilditch R. W., Howarth I. D., 2003, MNRAS, 339, 157

Hartman J. W., Bhattacharya D., Wijers R., Verbunt F., 1997, A\&A, 322, 477

Hewish A., Bell S. J., Pilkington J. D. H., Scott P. F., Collins R. A., 1968, Nature, 217,709

Hobbs G., Lorimer D. R., Lyne A. G., Kramer M., 2005, MNRAS, 360, 974

Johnston H. M., Kulkarni S. R., 1991, ApJ, 368, 504

Johnston S., 1994, MNRAS, 268, 595

Johnston S., Romani R., 2003, ApJ, 590, L95

Keith M. J., Jameson A., van Straten W., Bailes M., Johnston S., Kramer M., Possenti A., Bates S. D., Bhat N. D. R., Burgay M., Burke-Spolaor S., D'Amico N., Levin L., Milia S., Stappers B. W., 2010, ArXiv e-prints

Kim S., Staveley-Smith L., Dopita M. A., Freeman K. C., Sault R. J., Kesteven M. J., McConnell D., 1998, ApJ, 503, 674

Koerwer J. F., 2009, AJ, 138, 1

Lattimer J. M., Prakash M., 2001, ApJ, 550, 426

Livingstone M. A., Kaspi V. M., Gotthelf E. V., Kuiper L., 2006, ApJ, 647, 1286

Löhmer O., Mitra D., Gupta Y., Kramer M., Ahuja A., 2004, A\&A, 425, 569

Lorimer D. R., Bailes M., Dewey R. J., Harrison P. A., 1993, MNRAS, 263, 403 
Lorimer D. R., Bailes M., Harrison P. A., 1997, MNRAS, 289, 592

Lorimer D. R., Faulkner A. J., Lyne A. G., Manchester R. N., Kramer M., McLaughlin M. A., Hobbs G., Possenti A., Stairs I. H., Camilo F., Burgay M., D'Amico N., Corongiu A., Crawford F., 2006, MNRAS, 372, 777

Lorimer D. R., Kramer M., 2005, Handbook of Pulsar Astronomy. Cambridge University Press

Lyne A. G., Manchester R. N., 1988, MNRAS, 234, 477

Lyne A. G., Manchester R. N., Taylor J. H., 1985, MNRAS, 213, 613

Manchester R. N., Fan G., Lyne A. G., Kaspi V. M., Crawford F., 2006, ApJ, 649, 235

Manchester R. N., Hobbs G. B., Teoh A., Hobbs M., 2005, AJ, 129, 1993

Manchester R. N., Lyne A. G., Camilo F., Bell J. F., Kaspi V. M., D'Amico N., McKay N. P. F., Crawford F., Stairs I. H., Possenti A., Morris D. J., Sheppard D. C., 2001, MNRAS, 328, 17

Manchester R. N., Taylor J. H., 1977, Pulsars. Freeman, San Francisco

Maron O., Kijak J., Kramer M., Wielebinski R., 2000, in Kramer M., Wex N., Wielebinski R., eds, Pulsar Astronomy - 2000 and Beyond, IAU Colloquium 177 Pulsar spectra analysis. Astronomical Society of the Pacific, San Francisco, pp $227-228$

McConnell D., McCulloch P. M., Hamilton P. A., Ables J. G., Hall P. J., Jacka C. E., Hunt A. J., 1991, MNRAS, 249, 654

Narayan R., Ostriker J. P., 1990, ApJ, 352, 222

Narayan R., Vivekanand M., 1983, A\&A, 122, 45

Ostriker J. P., Gunn J. E., 1969, ApJ, 157, 1395

Pacini F., 1968, Nature, 219, 145

Press W. H., Teukolsky S. A., Vetterling W. T., Flannery B. P., 1992, Numerical Recipes: The Art of Scientific Computing, $2^{\text {nd }}$ edition. Cambridge University Press, Cambridge

Ransom S. M., Eikenberry S. S., Middleditch J., 2002, AJ, 124, 1788

Reynolds S. P., Borkowski K. J., Green D. A., Hwang U., Harrus I., Petre R., 2008, ApJ, 680, L41

Ridley J. P., Lorimer D. R., 2010, MNRAS, 404, 1081 
Ridley J. P., Lorimer D. R., 2010, MNRAS, 406, L80

Smits R., Kramer M., Stappers B., Lorimer D. R., Cordes J., Faulkner A., 2009, A\&A, 493, 1161

Smits R., Lorimer D. R., Kramer M., Manchester R., Stappers B., Jin C. J., Nan R. D., Li D., 2009, A\&A, 505, 919

Staveley-Smith L., Wilson W. E., Bird T. S., Disney M. J., Ekers R. D., Freeman K. C., Haynes R. F., Sinclair M. W., Vaile R. A., Webster R. L., Wright A. E., 1996, PASA, 13, 243

Tammann G. A., Löffler W., Schröder A., 1994, ApJS, 92, 487

Tauris T. M., Manchester R. N., 1998, MNRAS, 298, 625

Taylor J. H., Huguenin G. R., 1969, Nature, 221, 816

Taylor J. H., Manchester R. N., 1977, ApJ, 215, 885

Thorsett S. E., Chakrabarty D., 1999, ApJ, 512, 288

van der Marel R. P., 2001, AJ, 122, 1827

Wall J. V., Jenkins C. R., 2001, Practical Statistics for Astronomers. Cambridge University Press

Weltevrede P., Johnston S., 2008, MNRAS, 387, 1755

Weltevrede P., Johnston S., Espinoza C. M., 2010, ArXiv e-prints

Young M. D., Manchester R. N., Johnston S., 1999, Nature, 400, 848

Yusifov I., Küçük I., 2004, A\&A, 422, 545 


\section{Appendix A}

\section{An Open-source Approach to Pulsar Population Synthesis}

Following the initial version described by Lorimer et al. (2006), the source code used in this work is freely available at http://psrpop. sourceforge.net. The new features of the pulsar simulation package, PSRPOP, are described here, as well as the announcement of the availability of a website, http://psrpop.phys.wvu.edu, used to investigate past and future pulsar surveys.

The biggest new feature of the software package is the ability to evolve a pulsar's spin period in time. Currently, spin-down models from Faucher-Giguère \& Kaspi (2006) and Contopoulos \& Spitkovsky (2006) are available for use in the simulations. By having this evolution feature, a pulsar's life cycle can now be viewed from birth to death, and how its period changes with time can be observed. The program also has the ability to use various beaming models, luminosity laws, and alignment angle functions. These allow for further studying of the individual pulsars, and a more complete understanding of pulsar populations as a whole.

The PSRPOP website currently has the capability of surveying any of the eight model populations generated from Chapter 2 and future models will also be available for use. The user can "search" these model populations using previous surveys such as the Parkes Multibeam Survey, theoretical surveys such as one using the proposed Square Kilometer Array, or they can create their own survey. One of the main 


\section{PULSAR SURVEYS}
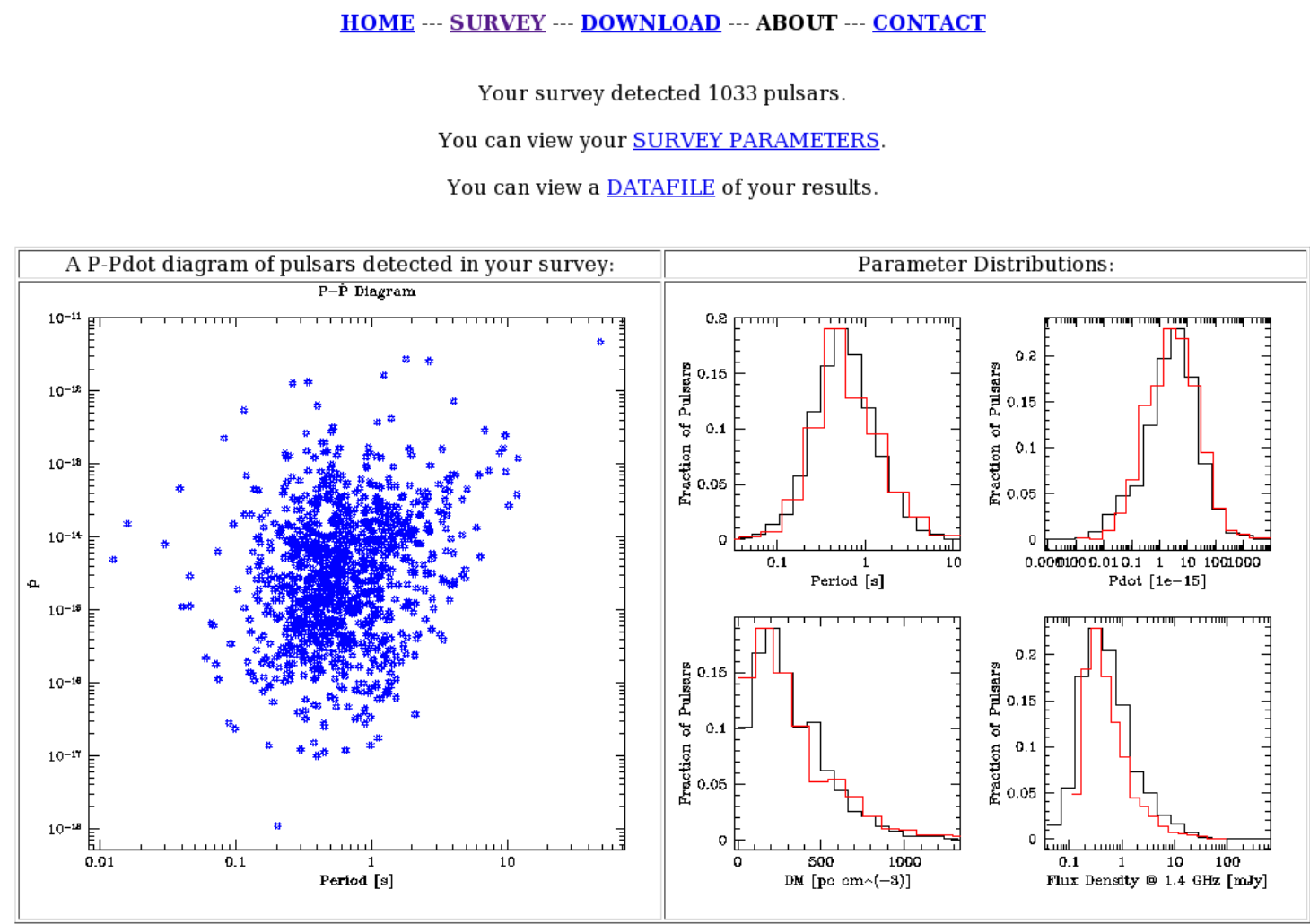

Figure A.1: The website output of the PSRPOP website. Here, the normal pulsar population was surveyed using the parameters from the Parkes Multibeam Survey. The detected pulsars are displayed in a $P-\dot{P}$ diagram, and the overall distribution of $P, \dot{P}, \mathrm{DM}$, and flux density of the simulated pulsars (in red) are shown in histogram form against the observed distributions (in black).

benefits of running a custom survey is the ability to predict yields of future surveys.

After surveying one of the pulsar populations, the website outputs the total number of pulsars detected, a $P-\dot{P}$ diagram, and a few comparison plots. These plots contain histograms of the pulsar properties detected in the survey that overlay histograms of the observed pulsar population. Some possible properties that can be shown in the histogram plots are pulse period, period derivative, dispersion measure, and flux density. Figure A.1 shows a sample output when the normal 
pulsar population is surveyed using the Parkes Multibeam Survey parameters. 


\section{Appendix B}

\section{A Search for the Youngest Pulsar in the Galaxy}

The young pulsar population in the Galaxy is not well understood, so searching for and finding young pulsars would yield answers to many uncertainties, such as their birth rates, luminosities, and beaming fractions. Since many pulsars are believed to have been formed in supernovae, supernova remnants (SNRs) are logical places in which to search for pulsars.

Supernova remnant G1.9 + 0.3 was discovered by Green \& Gull (1984). Later observations by Reynolds et al. (2008) using the Chandra X-ray Telescope (see Figure B.1) determined that the SNR was on the order of 100 years old. Currently, the youngest known pulsar in the Milky Way Galaxy is J1846-0258, which is slightly more than 700 years old (Gotthelf et al., 2000).

The area around G1.9+0.3 has been previously searched for pulsars in surveys (such as the Parkes Multibeam Survey), however, no periodic or bursting sources were discovered. Lower flux density limits at $1.4 \mathrm{GHz}$ of 0.3 and $450 \mathrm{mJy}$, respectively, were attained in those searches, meaning that the maximum $1.4 \mathrm{GHz}$

luminosity that a potential pulsar could have would be $20 \mathrm{mJy} \mathrm{kpc}^{2}$. Luminosities higher than this should have made the pulsar visible in these searches.

With the use of the GUPPI backend at the Green Bank Telescope (DuPlain et al., 2008), it is possible to improve the sensitivity of a pulsar search by at least an 


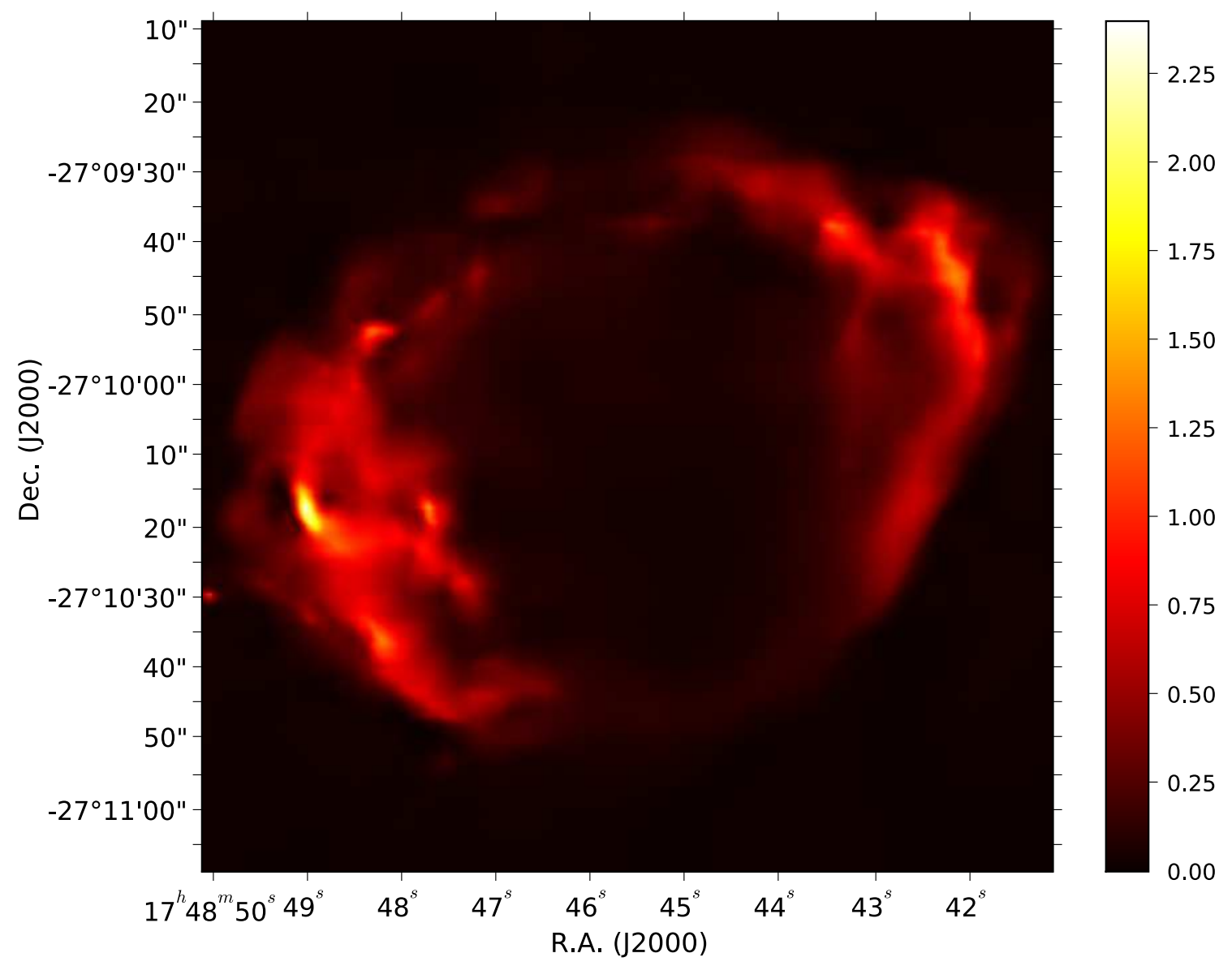

Figure B.1: The Chandra X-ray image showing SNR G1.9 + 0.3 (Reynolds et al., 2008).

order of magnitude over the Parkes Multibeam Survey due to its larger bandwidth and smaller frequency channels. This fact provided the motivation to undergo an additional search for a pulsar in G1.9+0.3.

Using the assumed distance to the SNR of $8.5 \mathrm{kpc}$ (Reynolds et al., 2008), the Cordes-Lazio NE2001 electron density model predicts a DM of $700 \mathrm{pc} \mathrm{cm}^{-3}$. The pulse broadening due to interstellar scattering at an observing frequency of 1.4 GHz is $14 \mathrm{~ms}$. This is a significant fraction of the pulsar period for many of the young pulsars in the Galaxy and would likely inhibit any detection at this frequency, 
including the Parkes Multibeam Survey. However, at a frequency of $5 \mathrm{GHz}$, the scattering is only around $50 \mu \mathrm{s}$. Higher frequencies would reduce the amount of scattering even further, but the steep spectral indices of pulsars (on average -1.8 , Maron et al. (2000)) would make observations at such high frequencies much less sensitive.

In July 2009, a search of SNR G1.9 + 0.3 took place at the Green Bank Telescope using the GUPPI backend. The C-band receiver, centered at a frequency of $5 \mathrm{GHz}$, was used for a single 7.5 hour observation. More than 130 DMs were searched out to a DM of $3000 \mathrm{pc} \mathrm{cm}^{-3}$. The minimum $\mathrm{S} / \mathrm{N}$ that was considered was 8 , which made the search sensitive down to a $1.4 \mathrm{GHz}$ luminosity limit of $6.9 \mathrm{mJy} \mathrm{kpc}^{2}$. This would make $70 \%$ of the known pulsars visible, however, no periodicities or single bursts were observed.

The lack of any detections in this search yields three main conclusions regarding the central compact object of SNR G1.9+0.3. First, it is possible that the object is not a pulsar. It could be a central compact object (CCO) and thus no periodic signals should be expected. Second, the object could be a pulsar that is beaming away from the Earth. Estimates from Tauris \& Manchester (1998) predict that only around $10 \%$ of all pulsars are beaming in the direction of Earth. Finally, it is possible that the object could be a pulsar that has a luminosity of less than $6.9 \mathrm{mJy} \mathrm{kpc}^{2}$, which would put it into a category along with $30 \%$ of all pulsars that would not have been visible with these observations. Further observations of SNR G1.9 +0.3 could be even more sensitive, and thus possibly discover the youngest pulsar in the Galaxy!

\footnotetext{
John $\mathrm{H}$. 